\title{
On a question of Igusa, II: Uniform asymptotic bounds for Fourier transforms in several variables
}

\author{
Ben Lichtin
}

\begin{abstract}
This paper shows that a nontrivial uniform decay estimate for complete exponential sums modulo $p^{r}$, determined by a polynomial map $\mathbf{P}=\left(P_{1}, P_{2}\right)$, follows from the existence of a 'good $\mathbf{P}$ decomposition' of $\mathbb{Z}_{p}^{n}$, a property that can be proved with geometric methods, and which was introduced in an earlier article by the present author.
\end{abstract}

\section{Introduction}

The origin of this paper is a question of Igusa [Igu78, p. 32]. This asked for an extension to several variables of his and Weil's work [Wei65] that connected the singularities of the local singular series, determined by a polynomial $P \in \mathbb{Z}_{p}\left[x_{1}, \ldots, x_{n}\right]$, to the decay behavior of its Fourier transform, which equals the normalized Gaussian sum (when evaluated at $a / p^{r}$ ):

$$
G\left(a / p^{r}\right) \underset{\operatorname{def}}{=} p^{-r n} \sum_{x \in\left(\mathbb{Z} / p^{r}\right)^{n}} e^{2 \pi i a P(x) / p^{r}}, \quad(a, p)=1 .
$$

Igusa used geometric methods to show the existence of a decay exponent $\alpha<0$ such that for any $\varepsilon>0, G\left(a / p^{r}\right)=O_{\varepsilon}\left(p^{r(\alpha+\varepsilon)}\right)$ as $r \rightarrow+\infty$. A geometric characterization of $\alpha$ was given in terms of a finite amount of numerical data, determined by an embedded resolution of singularities applied to each of the (finitely many) singular fibers of $\left.P\right|_{\mathbb{Z}_{p}^{n}}$. Given this data, one can then decide, in particular, whether the function $a / p^{r} \rightarrow G\left(a / p^{r}\right)$ belongs to $L^{1}\left(\mathbb{Q}_{p}\right)$. When $P$ has integral coefficients, $\alpha$ can be chosen independently of $p$.

A nontrivial application of the condition $\alpha<-2$, combined with a simple geometric property of the hypersurface $\{P=0\}$, is a 'generalized Poisson summation formula'. This is the basis of Igusa's theory of 'admissible representations', whose goal was to extend the Siegel-Weil theory to a larger class of representations of semi-simple groups. In addition, such a formula can be used [Igu76] to infer the existence of (nontrivial) integral points on the hypersurface defined by an invariant polynomial of the underlying group action. An important tool that can often be used to prove $\alpha<-2$ by purely geometric methods was a property that Igusa called 'condition $P^{\prime}$ ' [Igu78, p. 155].

An extension of this theory to $k(2 \leqslant k \leqslant n)$ variables should lead to nontrivial decay estimates as $r \rightarrow \infty$ for multivariate normalized Gaussian sums of the form

$$
G\left(\mathbf{a} / p^{r}\right)=p^{-r n} \sum_{x \in\left(\mathbb{Z} / p^{r}\right)^{n}} e^{2 \pi i\left(a_{1} P_{1}(x)+\cdots+a_{k} P_{k}(x)\right) / p^{r}}, \quad\left(p, a_{1}, \ldots, a_{k}\right)=1,
$$

that are uniform in $\mathbf{a}=\left(a_{1}, \ldots, a_{k}\right)$.

Received 19 February 2003, accepted in final form 4 December 2003, published online 1 December 2004.

2000 Mathematics Subject Classification 11L03, 11L07, 14B05, 14G20, 32S45.

Keywords: fiber integral, singular series, polynomial mapping, exponential sums.

This journal is (c) Foundation Compositio Mathematica 2005. 
Assuming that favorable geometric conditions are satisfied by the polynomial map $\mathbf{P}=$ $\left(P_{1}, \ldots, P_{k}\right)$, it should also be possible to show that a decay exponent $\alpha$ for $G\left(\mathbf{a} / p^{r}\right)$ satisfies the inequality $\alpha<-k-1$. A reasonably simple geometric analogue of condition $P$ should help to establish this estimate for $\alpha$. When this occurs, a multivariate generalized Poisson formula (over the adèles) would follow by a natural variant of Igusa's (and Weil's) local-global method. Applied, in particular, to the admissible representations classified in [Igu73] whose ring of invariants defines a variety of codimension at least 2 , this would then imply, among other properties, that nontrivial integral points lie on the variety. A recent work of Braverman and Kazhdan [BK00] has also described a second application of a multivariate Poisson formula.

On the other hand, the applicability of Igusa's work to deduce the presence of nontrivial rational points even on a smooth projective hypersurface of degree $d$ in $n=O\left(d^{c}\right)$ (for some $c$ ) variables, has not yet been realized and still appears to be quite difficult.

Igusa's work is based on the embedded resolution of singularities applied to the divisor defined by $P$. The main use of this theorem is the existence of a finite decomposition $\mathbb{Z}_{p}^{n}=\bigcup W_{j}$ into 'wedges', in each of which $P$ equals a monomial. An extension of his method to $k \geqslant 2$ polynomials is straightforward if an analogous finite decomposition of $\mathbb{Z}_{p}^{n}$ could be established, in each wedge $W$ of which the mapping $\mathbf{P}$ was a monomial map of the form $\mathbf{x} \in W \rightarrow\left(\mathbf{x}^{\mathbf{M}_{1}}, \ldots, \mathbf{x}^{\mathbf{M}_{k}}\right)$ such that the rank of the matrix whose rows are the $\mathbf{M}_{i}$ equals $k$. This, however, is too much to expect in general, as simple examples clearly indicate.

A more useful point of view allows base change maps of a restricted form as well as (local) blowing-up maps in the domain. In a preceding work [Lic00] (see also $\S 1$ ), the notion of a good $\mathbf{P}$ decomposition of $\mathbb{Z}_{p}^{n}$ was introduced and was used to give a reasonable generalization of Igusa's work in the first nontrivial case when $k=2$. Roughly speaking, a good $\mathbf{P}$ decomposition of $\mathbb{Z}_{p}^{n}$ for a map $\mathbf{P}=\left(P_{1}, P_{2}\right)$ decomposes $\mathbb{Z}_{p}^{n}$ as a finite union $\mathbb{Z}_{p}^{n}=\bigcup_{j} W_{j}$ of 'good $\mathbf{P}$ wedges' such that the Haar measure of any overlap $W_{i} \cap W_{j}, i \neq j$, equals zero. In turn, this decomposes the fiber integral (i.e. local singular series),

$$
t=\text { regular value of } \mathbf{P} \rightarrow F(t)=\int_{\{\mathbf{P}=t\} \cap \mathbb{Z}_{p}^{n}}\left|d x / d P_{1} \wedge d P_{2}\right|
$$

into the sum of contributions $F(t)=\sum_{j} F_{W_{j}}(t)$ from each wedge. A basic point is that the goodness of $W_{j}$ leads to an explicit description of the singularities (if any) of $F_{W_{j}}(t)$ along the critical values in $\mathbf{P}\left(W_{j}\right)$, at least after a suitable base change.

The main result of this paper applies this idea to bound uniformly and nontrivially the Fourier transform of $F(t)$; that is the Gaussian sums $G\left(\mathbf{a} / p^{r}\right)$, as $r \rightarrow \infty$, as follows.

Theorem A. Let $\mathbf{P}: \mathbb{Q}_{p}^{n} \rightarrow \mathbb{Q}_{p}^{2}$ be a polynomial mapping for which a good $\mathbf{P}$ decomposition $\mathbb{Z}_{p}^{n}$ exists. Then there exists $\alpha=\alpha(\mathbf{P})<0$, such that

$$
G\left(\mathbf{a} / p^{r}\right)=O_{\varepsilon}\left(p^{r(\alpha+\varepsilon)}\right) \quad r \rightarrow \infty
$$

uniformly over all $\mathbf{a}=\left(a_{1}, a_{2}\right)$ such that $\left(p, a_{1}, a_{2}\right)=1$.

A good $\mathbf{P}$ decomposition $\mathbb{Z}_{p}^{n}=\bigcup_{j} W_{j}$ reduces the global problem of estimating the decay of $G\left(\mathbf{a} / p^{r}\right)$ to the local one of estimating the decay of the Fourier transform $F_{W_{j}}^{*}$ of each $F_{W_{j}}$. Since the singularities of each $F_{W_{j}}$ are well understood, the difficulty is to prove the existence of a local decay exponent $\alpha\left(W_{j}\right)<0$ for $F_{W_{j}}^{*}\left(\mathbf{a} / p^{r}\right)$ as $r \rightarrow \infty$. It then suffices to set $\alpha=\max _{j} \alpha\left(W_{j}\right)$ to prove Theorem A. An important point is that each $\alpha\left(W_{j}\right)$ is given explicitly in terms of a local polygon that encodes the geometric features of $W_{j}$. In this sense, it is fair to say that $\alpha$ is effectively computable using the geometry of $\mathbf{P}$. This is a reasonable analogue of Igusa's geometric characterization of the decay exponent $\alpha$ when $k=1$. It also suffices to work over $\mathbb{Q}_{p}$ since the calculations and proofs extend straightforwardly to any finite extension of $\mathbb{Q}_{p}$. 


\section{B. LICHTIN}

The proof of the local estimate for $F_{W_{j}}^{*}\left(\mathbf{a} / p^{r}\right)$ is given in $\S 3$. Sections 1 and 2 contain required preliminaries and helpful reductions for the proof. Section 4 discusses the appropriate extension of condition $P$, which is called condition $P^{(2)}$. Two short appendices conclude the paper.

At present there are classes of polynomial maps $\mathbf{P}$ for which a good $\mathbf{P}$ decomposition of $\mathbb{Z}_{p}^{n}$ is known to exist. Some of these are as follows (recall that the critical locus of $\mathbf{P}$ is the set of common zeros of all $2 \times 2$ minors of the Jacobian matrix of $\mathbf{P}$ ):

$$
\mathcal{C}_{1}=\left\{\mathbf{P}: \mathbb{Q}_{p}^{n} \rightarrow \mathbb{Q}_{p}^{2}: n=2,3 \text { and } \mathbf{P} \text { is dominant }\right\}
$$

$\mathcal{C}_{2}=\left\{\mathbf{P}: \mathbb{Q}_{p}^{n} \rightarrow \mathbb{Q}_{p}^{2}: n \geqslant 3\right.$, the critical locus of $\mathbf{P}$ is a nonsingular curve outside the origin and each $P_{i}$ is homogeneous and nonsingular outside the origin\};

$$
\mathcal{C}_{3}=\left\{\mathbf{P}: \mathbb{Q}_{p}^{2 n} \rightarrow \mathbb{Q}_{p}^{2}: P_{1}(\beta, x)=\sum_{1}^{n} \beta_{i} x_{i} \text { and } P_{2}=P_{2}(x)\right. \text { is homogeneous and nonsingular }
$$

outside the origin $\}$.

The proofs for $\mathcal{C}_{3}$ and $\mathcal{C}_{1}$ when $n=2$ are given in [Lic00]. The proof for $\mathcal{C}_{1}$ when $n=3$ is nontrivial and given in [Cut02]. The proof for $\mathcal{C}_{2}$ given in [Lic00, $\left.\S 3\right]$ was incomplete. A correct proof is given in [Lic03]. A very interesting problem is to extend the method of that paper to treat pairs of homogeneous $\mathbf{P}$ as in $\mathcal{C}_{2}$ for which the critical locus has larger dimension but remains nonsingular outside the origin. A second interesting problem is to extend the method to pairs for which the critical locus is also singular (in some controlled way) outside the origin. This ought to be possible, at the least, if $\mathbf{P}$ belongs to a given Thom-Boardman class.

In contrast to the case $k=2$, the situation for $\mathbf{P}: \mathbb{Q}_{p}^{n} \rightarrow \mathbb{Q}_{p}^{k}, k \geqslant 3$, is more complicated. More precisely, the correct class of permissible base change maps, by means of which one can expect to improve $\mathbf{P}$ in some open neighborhood of any critical point (see (1.2a)-(1.2c)) will be larger. As a result, no good $\mathbf{P}$ decomposition of $\mathbb{Z}_{p}^{n}$ has yet been proved to exist for any reasonably interesting class of $\mathbf{P}$. Although there does not appear to be any fundamental obstruction to such a proof, the situation is more intricate because several additional types of base change maps are a priori possible once $k>2$ (see Remark 1.4(iv)). After this is worked out, the methods of this paper should extend straightforwardly and establish Theorem A for such $\mathbf{P}$.

Explicit values for $\alpha$ can be given for pairs in $\mathcal{C}_{2} \cup \mathcal{C}_{3}$ as follows:

(i) if $\mathbf{P} \in \mathcal{C}_{2}$ and $d_{i}=\operatorname{deg} P_{i}$, then one can choose $\alpha=\max \left\{-n / d_{1},-n / d_{2},-(n-2) / 2\right\}$;

(ii) if $\mathbf{P} \in \mathcal{C}_{3}$ and $d=\operatorname{deg} P_{2}$, then $\alpha=-n / d$.

This follows from an explicit parametrization of each wedge in a good $\mathbf{P}$ decomposition, the proof of Theorem A and the application of condition $P^{(2)}$ defined in $\S 4$ as an analog of condition $P$. The formula for $\alpha$ in (i) will be used in forthcoming work to prove a generalized Poisson formula for pairs of forms in $\mathcal{C}_{2}$ when $n>3 \cdot \max \left\{d_{1}, d_{2}\right\}$ if $d_{1}$ or $d_{2}>2$, or $n>8$ if $d_{1}=d_{2}=2$. In [Lic04], the analog of Theorem A over $\mathbb{R}$ is proved.

Using a very different approach, based on model theory, Cluckers [Clu04] has recently proved a very general result. He showed the existence of a uniform and nontrivial decay rate for the Gaussian sums determined by any restricted power series mapping $\mathbf{P}: \mathbb{Z}_{p}^{n} \rightarrow \mathbb{Z}_{p}^{k}(k \geqslant 2)$ whose image has nonempty interior. This is as general a result as one could probably hope to prove. His argument relies on an earlier result of Denef [Den00] that used model theory to deduce the general behavior and form of the singularities of the fiber integral along the set of critical values of $\mathbf{P}$. On the other hand, the method of Cluckers-Denef is not yet constructive. Nor does it establish a property analogous to condition $P^{(2)}$. This is crucial for deducing the existence of a decay rate better than $\alpha=-1$, which is important for applications to global problems. 


\section{ON A QUESTION OF IGUSA, II}

Taken together, these results (and the many outstanding questions) will hopefully encourage further work on this subject, the goal of which should be to find additional classes of maps $\mathbf{P}$ for which good (and reasonably explicit) bounds for the decay rate of the sums (0.2) can be given. For this, it seems safe to say, geometric methods appear to be essential.

Notation. Throughout the paper, $\varepsilon$ denotes any positive number that is 'sufficiently small' (also indicated by $\varepsilon \ll 1$ ). That is, $\varepsilon$ is smaller than some suitable constant whose precise value may vary in different estimates. The notation $O_{\varepsilon}$ has the standard meaning that for any sufficiently small $\varepsilon$, the constant in the upper bound depends only on $\varepsilon$. The complex and $p$-adic norms are both denoted by $|\cdot|$, and context will indicate which is intended. (The Fourier transforms defined in $\S 1$ are, of course, estimated as complex numbers.) The order of a $p$-adic number $x$ is written ord $x$. Further, $\log |x|$ denotes $\log$ to base $p$. So, one can identify it with $-\operatorname{ord} x$. The group of units is written $\mathcal{U}_{p}$. The coset of an element $\mathbf{a} \in \mathbb{Q}_{p}^{n}$ modulo $p^{M}$ is denoted by $[\mathbf{a}]_{M}$ (and equals $\left.\mathbf{a}+\left(p^{M}\right)^{(n)}\right)$. The critical locus of $\mathbf{P}$ is denoted by $\Sigma_{\mathbf{P}}$. If $x_{1}, \ldots, x_{r}$ are coordinates and $\mathbf{N}=\left(N_{1}, \ldots, N_{r}\right)$ is an integral vector, then $\mathbf{x}^{\mathbf{N}}={ }_{\text {def }} \prod_{i=1}^{r} x_{i}^{N_{i}}$. Roman fonts are used to denote subsets of $\mathbb{Q}_{p}^{n}$, while script fonts denote the set of $p$-adic orders of the elements of the set.

\section{Required preliminaries}

This section summarizes ideas from [Lic00] that are needed to prove Theorem A.

\subsection{Good P decomposition}

Let $\boldsymbol{x} \in \mathbb{Q}_{p}^{n}$ and let $(f, g):(U(\boldsymbol{x}), \boldsymbol{x}) \rightarrow\left(\mathbb{Q}_{p}^{2}, \mathbf{0}\right)$ be an analytic map defined in the (compact and open) neighborhood of $\boldsymbol{x}$.

Definition 1.1. The point $\boldsymbol{x}$ is good for $(f, g)$ if there exist local ( $p$-adic analytic) coordinates $\boldsymbol{z}=\left(z_{1}, \ldots, z_{n}\right)$ defined on a neighborhood $U^{\prime}(\boldsymbol{x}) \subset U(\boldsymbol{x})$ such that

$$
\begin{gathered}
f(\boldsymbol{z})=\prod_{i=1}^{R} z_{i}^{N_{i}} u_{1}, \quad g(\boldsymbol{z})=\prod_{i=1}^{R} z_{i}^{M_{i}} u_{2}, \quad u_{1}, u_{2} \text { units on } U^{\prime}(\boldsymbol{x}) \\
\text { and } A(\boldsymbol{x})=\left(\begin{array}{llll}
N_{1} & N_{2} & \cdots & N_{R} \\
M_{1} & M_{2} & \cdots & M_{R}
\end{array}\right) \text { has rank }=2 .
\end{gathered}
$$

(Only nonzero columns of $A(\boldsymbol{x})$ are indicated. By definition, the coordinates map $\boldsymbol{x}$ to $\mathbf{0}$.). If (1.1) is not satisfied, then $\boldsymbol{x}$ is a bad point.

Remarks 1.2.

(i) If $\boldsymbol{x}$ is a noncritical point of $(f, g)$, then the implicit function theorem ensures that it is a good point with $A(\boldsymbol{x})$ the $2 \times 2$ identity matrix.

(ii) If (1.1) is satisfied, then one may assume that $U(\boldsymbol{x})$ and the coordinates $\boldsymbol{z}$ are chosen so that $f(\boldsymbol{z})$ and $g(\boldsymbol{z})$ are pure monomials (no unit factor appears) defined on $U(\boldsymbol{x})$. It is as a local monomial map that a mapping will be written in some neighborhood of a good point.

(iii) The point $\boldsymbol{x}$ is bad, in particular, if $f$ and $g$ can both be written locally as the product of a monomial and local unit, but the rank of the matrix $A(\boldsymbol{x})$ equals 1 . It is, however, also convenient to say that $\boldsymbol{x}$ is bad if at least one of the components of $(f, g)$ is not the product of a monomial and local unit.

Definition 1.3. A permissible modification of $(f, g):(U(\boldsymbol{x}), \boldsymbol{x}) \rightarrow\left(\mathbb{Q}_{p}^{2},(0,0)\right)$ in $U(\boldsymbol{x})$ is a transformation $\zeta:(F, G) \rightarrow(f, g)$, defined as follows. Either $(F, G)=(f, g)$ and the map is the identity, or there exist a positive integer $\gamma$, a nonzero constant $c_{0}$ and a nonconstant $p$-adic analytic 


\section{B. LICHTIN}

function $\psi=\psi(w)$, all depending on $\boldsymbol{x}$, such that on $U(\boldsymbol{x})$ either

$$
g=c_{0} F^{\gamma}, \quad f=G+\psi(F),
$$

or

$$
f=c_{0} F^{\gamma}, \quad g=G+\psi(F) .
$$

A permissible modification $(F, G) \rightarrow(f, g)$, defined on a neighborhood $U(\boldsymbol{x})$, is an amelioration at $\boldsymbol{x}$ if $\boldsymbol{x}$ is a bad point for $(f, g)$ and a good point for $(F, G)$.

Given $\mathbf{t}=\left(t_{1}, t_{2}\right)$ (respectively $\boldsymbol{\tau}=\left(\tau_{1}, \tau_{2}\right)$ ) as coordinates on $\left.\operatorname{Im}(f, g)\right|_{U(\boldsymbol{x})}$ (respectively $\left.\left.\operatorname{Im}(F, G)\right|_{U(\boldsymbol{x})}\right)$, there are induced 'base change' maps:

$$
\zeta: \boldsymbol{\tau} \rightarrow \mathbf{t}= \begin{cases}\left(\tau_{2}+\psi\left(\tau_{1}\right), c_{0} \tau_{1}^{\gamma}\right) & \text { if }(1.2 \mathrm{a}) \\ \left(c_{0} \tau_{1}^{\gamma}, \tau_{2}+\psi\left(\tau_{1}\right)\right) & \text { if }(1.2 \mathrm{~b}) .\end{cases}
$$

Remarks 1.4.

(i) For this paper, it is important to deduce the decay of the exponential sum $G(\boldsymbol{\lambda})$ (when $\boldsymbol{\lambda}=$ $\left.\mathbf{a} / p^{r},(p, \mathbf{a})=1\right)$ in terms of the parameter $|\boldsymbol{\lambda}|=\max \left\{\left|\lambda_{1}\right|,\left|\lambda_{2}\right|\right\}$. By insisting on (1.2a) as the form for each permissible modification, a uniform and nontrivial decay estimate in $|\boldsymbol{\lambda}|$ is proved in the cone $\left|\lambda_{2}\right| \leqslant\left|\lambda_{1}\right|$. If, however, (1.2b) is the form, then the same argument shows uniform and nontrivial decay in $|\boldsymbol{\lambda}|$ within the cone $\left|\lambda_{1}\right| \leqslant\left|\lambda_{2}\right|$.

It is therefore useful to distinguish the particular parametrizations in (1.2a) $-(1.2 \mathrm{c})$. For $k=1,2$, a permissible modification $\zeta:(F, G) \rightarrow(f, g)$ is said to be of type 1 (respectively type 2) if $\zeta$ is defined by (1.2a) (respectively $(1.2 \mathrm{~b}))$. One then also writes $\zeta_{k}:\left(F_{k}, G_{k}\right) \rightarrow(f, g)$ or $\left(F_{k}, G_{k}\right) \rightarrow(f, g)$.

(ii) Assume that $\boldsymbol{x}$ is a bad point for $(f, g)$ as in the first part of Remark 1.2(iii), and $\{i, k\}=\{1,2\}$. If an amelioration $\zeta_{k}:\left(F_{k}, G_{k}\right) \rightarrow(f, g)$ is defined on $U(\boldsymbol{x})$, then there also exists an amelioration $\zeta_{i}:\left(F_{i}, G_{i}\right) \rightarrow(f, g)$ at $\boldsymbol{x}$ that is defined on a possibly smaller $U(\boldsymbol{x})$. The elementary proof of this assertion is given in Appendix B.

(iii) Due to an unfortunate oversight, an additional condition was added incorrectly in [Lic03] to the defining property for a permissible modification. That third property (which required that $F$ divides $G$ ) is only pertinent when it is the case that both $f$ and $g$ are locally the product of a monomial and unit at the bad point $\boldsymbol{x}$ and the two monomials are dependent. Indeed, the discussion in [Lic03] exhibited several cases in which the divisibility of $G$ by $F$ was not possible, but a map $(F, G) \rightarrow(f, g)$ was nonetheless identified as being a permissible modification (see, e.g., [Lic03, 2.3.2]), in the sense that the basic form of (1.2a) or (1.2b) was satisfied. In these cases, this extra property was not needed for the subsequent discussion to apply, as a careful reading clearly indicates. The author regrets any confusion that may have ensued.

(iv) To illustrate the additional complexity that occurs when $k>2$, assume that $\boldsymbol{x}$ is bad (for $(f, g, h))$ as in (ii). Then $\operatorname{rank} A(\boldsymbol{x})=1$ or 2 . The form of any permissible modification $\zeta:(F, G, H) \rightarrow(f, g, h)$ will depend on the value of this rank. If the rank $=1$, then a reasonable definition for $\zeta$ should be given by

$$
\zeta:(F, G, H) \rightarrow\left(c_{0} F^{\delta}, \psi_{1}(F)+G, \psi_{2}(F)+H\right)=(f, g, h), \quad \psi_{1}(w), \psi_{2}(w) \text { analytic at } 0 .
$$

If the rank $=2$, then $\zeta$ should be given by

$$
\zeta:(F, G, H) \rightarrow\left(c_{0} F^{\delta_{1}}, c_{1} G^{\delta_{2}}, \psi(F, G)+H\right)=(f, g, h), \quad \psi(w) \text { analytic at } 0 .
$$

Of course, both possibilities would hold modulo permutations of the components $f, g, h$ as in $(1.2 \mathrm{a})-(1.2 \mathrm{c})$. 


\section{ON A QUESTION OF IGUSA, II}

Definition 1.5. Given the map $\mathbf{P}$, point $\mathbf{x} \in \mathbb{Z}_{p}^{n}$ and neighborhood $U(\mathbf{x})$, a good $\mathbf{P}$ wedge is a subset $W$ of $U(\mathbf{x})$ of the form $W=\theta U(\boldsymbol{x})$, where $\theta:(U(\boldsymbol{x}), \boldsymbol{x}) \rightarrow(U(\mathbf{x}), \mathbf{x})$ is either an analytic isomorphism, or a permissible transformation (i.e. composition of finitely many local blowing-ups with nonsingular analytic center), such that one of the following holds:

(i) the point $\boldsymbol{x}$ is good for $(f, g)={ }_{\operatorname{def}} \mathbf{P} \circ \theta-\mathbf{P}(\mathbf{x})$; or

(ii) the point $\boldsymbol{x}$ is bad for $(f, g)$, and there exist type $k$ ameliorations $\zeta_{k}:\left(F_{k}, G_{k}\right) \rightarrow(f, g)$ on $U(\boldsymbol{x})$ for each $k=1,2$.

The key idea is the existence of a good $\mathbf{P}$ decomposition for $\mathbb{Z}_{p}^{n}$ (any compact set will suffice, but for this paper, only $\mathbb{Z}_{p}^{n}$ is of interest).

Definition 1.6. A good $\mathbf{P}$ decomposition of $\mathbb{Z}_{p}^{n}$ consists of finitely many good $\mathbf{P}$ wedges $\left\{W_{i}\right\}_{i}$ such that:

(i) $\bigcup_{i} W_{i}=\mathbb{Z}_{p}^{n}$

(ii) for any $i \neq j, W_{i} \cap W_{j} \subset \Sigma_{\mathbf{P}}$.

Let $W=\theta U(\boldsymbol{x})$ be a good $\mathbf{P}$ wedge and assume $\mathbf{x} \in \Sigma_{\mathbf{P}}$. Since $\theta$ is permissible, the divisor, defined in $U(\boldsymbol{x})$ by its Jacobian determinant and denoted below by $d \theta$, will be a normal crossing divisor contained in the exceptional divisor of $\{f g=0\}$ if Definition 1.5(i) holds (respectively $\left\{F_{k} G_{k}=0\right\}$ if Definition 1.5(ii) holds). Thus, if the former (respectively latter) condition occurs, then $d \theta$ will equal the product of a monomial and local unit in the local coordinates $\boldsymbol{z}$ in which $(f, g)$ (respectively $\left(F_{k}, G_{k}\right)$ ) is a monomial map. In this case the following definition will be needed.

Definition 1.7. If Definition 1.5(i) holds and $A(\boldsymbol{x})$ is the matrix (1.1) of multiplicities for $(f, g)$, define

$$
\mathbf{A}(\boldsymbol{x})=\left(\begin{array}{llll}
N_{1} & N_{2} & \cdots & N_{R} \\
M_{1} & M_{2} & \cdots & M_{R} \\
\mu_{1} & \mu_{2} & \cdots & \mu_{R}
\end{array}\right) \quad \text { if } d \theta(\boldsymbol{z})=\prod_{i=1}^{R} z_{i}^{\mu_{i}-1} \cdot \text { (local unit). }
$$

If Definition 1.5(ii) holds, define the matrix $\mathbf{A}_{k}(\boldsymbol{x})$ by setting its first two rows to be the multiplicities of $\left(F_{k}, G_{k}\right)$ and the third row to be $\left(\mu_{1}, \ldots, \mu_{R}\right)$.

Given a $3 \times R$ matrix $\mathbf{A}=\mathbf{A}(\boldsymbol{x})$ or $\mathbf{A}_{k}(\boldsymbol{x})$ as above, one can then define a local polygon $\Gamma=\Gamma(\boldsymbol{x})$ in the third quadrant by setting

$$
\Gamma=\partial\left(\bigcap_{i=1}^{R}\left\{\left(\sigma_{1}, \sigma_{2}\right) \in(-\infty, 0]^{2}: N_{i} \sigma_{1}+M_{i} \sigma_{2} \geqslant-\mu_{i}\right\}\right) .
$$

There are at most two faces of $\Gamma$ that intersect the $\sigma_{1}, \sigma_{2}$ coordinate axes in exactly one point that does not equal the origin. Call these points the axis intercepts of $\Gamma$. Set

$$
\sigma(\Gamma)=\max _{i}\left\{\sigma_{i} \text {-axis intercept of } \Gamma\right\} .
$$

When emphasis of the dependance on $W$ and/or $k$ is warranted, the polygon is written $\Gamma(W)$ or $\Gamma_{k}(W)$.

\subsection{The singularities of the fiber integral and asymptotic monomials}

The definition of the fiber integral of $\mathbf{P}$ in the introduction uses the measure $\left|d x / d P_{1} \wedge d P_{2}\right|$ induced on $\{\mathbf{P}=t\}, t$ a regular value of $\mathbf{P}$, by the Leray differential form. This gives an integral representation for the local singular series of $\mathbf{P}$, the map $t \rightarrow p^{-r(n-2)} \cdot \#\left\{x \bmod p^{r}: \mathbf{P}(x) \equiv t \bmod p^{r}\right\}$ (see [Yam83]). If $W$ is a good $\mathbf{P}$ wedge, define the 'local contribution to the fiber integral' (from $W$ ) 


\section{B. LICHTIN}

as

$$
F_{W}(t)=\int_{\{\mathbf{P}=t\} \cap W}\left|d x / d P_{1} \wedge d P_{2}\right|, \quad t \text { a regular value of } \mathbf{P} .
$$

It can of course equal zero. Given a good $\mathbf{P}$ decomposition $\mathbb{Z}_{p}^{n}=\bigcup_{i} W_{i}$, the fact that the intersection of the wedges is confined to the singular locus, which is of Haar measure zero, implies

$$
F(t)=\sum_{i} F_{W_{i}}(t)
$$

Let $W$ be a good $\mathbf{P}$ wedge with presentation $W=\theta U(\boldsymbol{x})$, where $\theta(\boldsymbol{x})=\mathbf{x}$. Setting $(f, g)=$ $\mathbf{P} \circ \theta-\mathbf{P}(\mathbf{x})$, it follows that

$$
\begin{aligned}
F_{W}(t) & =\int_{\{\mathbf{P} \circ \theta-\mathbf{P}(\mathbf{x})=t-\mathbf{P}(\mathbf{x})\} \cap U(\boldsymbol{x})}\left|\theta^{*} d x / d\left(P_{1} \circ \theta\right) \wedge d\left(P_{2} \circ \theta\right)\right| \\
& =\int_{\{(f, g)=\mathbf{t}\} \cap U(\boldsymbol{x})}\left|\theta^{*} d x / d f \wedge d g\right| \underset{\text { def }}{=} \mathbf{F}_{W}(\mathbf{t}), \quad \text { where } \mathbf{t} \underset{\text { def }}{=} t-\mathbf{P}(\mathbf{x}) .
\end{aligned}
$$

If $\boldsymbol{x}$ is a good point for $(f, g)$, then an explicit description for $\mathbf{F}_{W}(\mathbf{t})$ can be given, if $|\mathbf{t}| \ll 1$, as a finite sum of 'asymptotic monomials' in $\mathbf{t}$ (see [Lic00, Theorem 6.11]). If $\boldsymbol{x}$ is bad for $(f, g)$ and $\left(F_{k}, G_{k}\right) \rightarrow(f, g)$ is a type $k$ amelioration, then a similar description can be given if $|\boldsymbol{\tau}| \ll 1$ is a regular value of $\left(F_{k}, G_{k}\right)$, for the integral

$$
\mathcal{F}_{W, k}(\boldsymbol{\tau}) \underset{\text { def }}{=} \int_{\left\{\left(F_{k}, G_{k}\right)=\boldsymbol{\tau}\right\}}\left|\theta^{*} d x / d F_{k} \wedge d G_{k}\right| .
$$

In this case, the asymptotic monomials are functions of $\boldsymbol{\tau}$. It will be convenient to summarize the construction here.

Given the good point $\boldsymbol{x}$ for $(f, g)$ with matrix $\mathbf{A}(\boldsymbol{x})$ as in (1.3a) and (1.3b), define

$$
\begin{gathered}
\mathcal{L}_{i}=\left\{\left(\sigma_{1}, \sigma_{2}\right) \in \mathbb{R}^{2}: N_{i} \sigma_{1}+M_{i} \sigma_{2}=-\mu_{i}\right\} \text { for each } i, \\
\mathcal{I}_{0}=\left\{\{i<j\} \subset\{1, \ldots, R\}: \operatorname{rank}\left(\begin{array}{ll}
N_{i} & N_{j} \\
M_{i} & M_{j}
\end{array}\right)=2\right\} .
\end{gathered}
$$

Corresponding to each $\iota=\{i, j\} \in \mathcal{I}_{0}$, define the point, polygon and lattice (see Figure 1 ):

$$
\begin{gathered}
v_{\iota}=\mathcal{L}_{i} \cap \mathcal{L}_{j}=\left(v_{1}, v_{2}\right), \\
\Gamma_{\iota}=\partial\left(\bigcap_{u \in \iota}\left\{\left(\sigma_{1}, \sigma_{2}\right) \in(-\infty, 0]^{2}: N_{u} \sigma_{1}+M_{u} \sigma_{2} \geqslant-\mu_{u}\right\}\right), \\
\mathcal{C}_{\iota}=\left\langle\left(N_{i}, M_{i}\right),\left(N_{j}, M_{j}\right)\right\rangle_{\mathbb{Z}_{+} .}
\end{gathered}
$$

Write $\mathcal{C}_{\iota}^{*}$ to denote either $\mathcal{C}_{\iota}$ or one of the two sublattices spanned by $\left(N_{i}, M_{i}\right)$ or $\left(N_{j}, M_{j}\right)$.

Entirely similar constructions are made for each $\left(F_{k}, G_{k}\right)$ when $\boldsymbol{x}$ is bad for $(f, g)$. The data are then defined in terms of the matrix $\mathbf{A}_{k}(\boldsymbol{x})$.

Example. Suppose that $d \geqslant 4, n \geqslant 3$ and

$$
\mathbf{A}(\boldsymbol{x})=\left(\begin{array}{cccc}
d & 1 & 2 & 1 \\
d & 3 & 6 & 0 \\
n & 2(n-2) & 3(n-2)+1 & 1
\end{array}\right) .
$$

Then $\mathcal{I}_{0}=\{\{1,2\},\{1,3\},\{1,4\},\{2,4\},\{3,4\}\}$. Given $\iota=\{1,2\}$, then $\mathcal{C}_{\iota}=\langle(d, d),(1,3)\rangle$, and $v_{\iota}=$ $(n-2-3 n / 2 d,-(n-2)+n / 2 d)$. It follows that $v_{\iota}$ is only in the third quadrant if $n=3$, and then shifts to the fourth quadrant once $n \geqslant 4$. Thus, $\Gamma_{\iota}$ has two sides if and only if $n=3$. Otherwise $\Gamma_{\iota}$ has one side that lies on the line $d s_{1}+d s_{2}=-n$. 


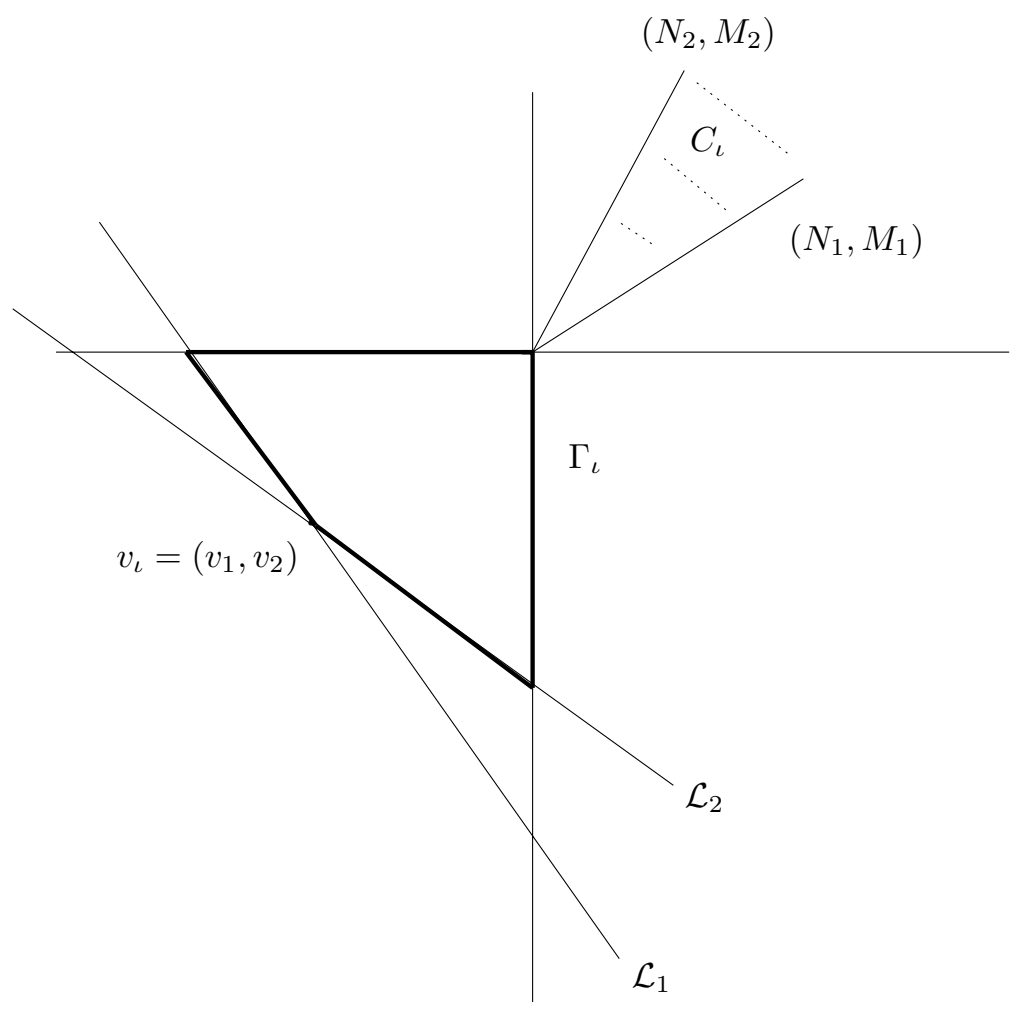

FiguRE 1.

Set $\chi=\left(\chi_{1}, \chi_{2}\right): \mathcal{U}_{p}^{2} \rightarrow S^{1} \times S^{1}$ to denote a pair of characters of $\mathcal{U}_{p}$.

Definition 1.8. Assume that $W=\theta U(\boldsymbol{x})$ is a good $\mathbf{P}$ wedge, and that $\boldsymbol{x}$ is a good point for $(f, g)=\mathbf{P} \circ \theta-\mathbf{P} \circ \theta(\boldsymbol{x})$. An asymptotic monomial for $\mathbf{F}_{W}(\mathbf{t})$ is a function defined as follows. Given $\iota \in \mathcal{I}_{0}, \mathbf{M}, \mathbf{b}=\left(b_{1}, b_{2}\right) \in \mathbb{Z}_{+}^{2}$ and pair $\boldsymbol{\chi}$, set $\boldsymbol{\omega}=(\iota, \mathbf{M}, \mathbf{b}, \boldsymbol{\chi})$ and define (outside $\left\{t_{1} t_{2}=0\right\}$ )

$$
\mathcal{M}_{\boldsymbol{\omega}}(\mathbf{t})= \begin{cases}\chi_{1}\left(\operatorname{act} t_{1}\right) \chi_{2}\left(\operatorname{act} t_{2}\right)\left|t_{1}\right|^{-v_{1}-1}\left|t_{2}\right|^{-v_{2}-1} \log ^{b_{1}}\left|t_{1}\right| \log ^{b_{2}}\left|t_{2}\right|_{\left.I m(f, g)\right|_{W}} & \text { if ord } \mathbf{t} \in \mathbf{M}+\mathcal{C}_{\iota}^{*} \\ 0 & \text { otherwise. }\end{cases}
$$

If $\boldsymbol{x}$ is bad for $(f, g)$ and $\left(F_{k}, G_{k}\right) \rightarrow(f, g)$ is a type $k$ amelioration at $\boldsymbol{x}$, an asymptotic monomial for $\mathcal{F}_{W, k}(\boldsymbol{\tau})$ is a monomial $\mathcal{M}_{\boldsymbol{\omega}}(\boldsymbol{\tau})$ whose form (in $\left.\tau_{1}, \tau_{2}\right)$ is as above, and $v, \iota, \mathcal{C}_{\iota}^{*}$ are defined as above by using the entries of $\mathbf{A}_{k}(\boldsymbol{x})$. Of course, in this case, $\boldsymbol{\tau}$ is restricted to $\left.\operatorname{Im}\left(F_{k}, G_{k}\right)\right|_{W}-\left\{\tau_{1} \tau_{2}=0\right\}$, and the monomial is not identically zero if and only if ord $\boldsymbol{\tau} \in \mathbf{M}+\mathcal{C}_{\iota}^{*}$.

Remark 1.9. To say, for example, that $\mathbf{F}_{W}(\mathbf{t})$ is a sum of finitely many asymptotic monomials if $\mathbf{t}$ is a regular value of $(f, g)$ and $|\mathbf{t}| \ll 1$ (see also [Lic00, pp. 72-73]), simply means the existence of a finite set $\mathcal{Y}_{W}=\left\{\boldsymbol{\omega}_{i}\right\}_{i=1}^{N}$, such that if $|\mathbf{t}| \ll 1$ and $\left.\mathbf{t}(=$ a regular value $) \in \operatorname{Im}(f, g)\right|_{W}$, then

$$
\mathbf{F}_{W}(\mathbf{t})=\sum_{i=1}^{N} c_{\boldsymbol{\omega}_{i}} \mathcal{M}_{\boldsymbol{\omega}_{i}}(\mathbf{t}), \quad c_{\boldsymbol{\omega}_{i}} \neq 0 \in \mathbb{Q}_{p}
$$

If $\boldsymbol{x}$ is bad for $(f, g)$ and $\zeta_{k}:\left(F_{k}, G_{k}\right) \rightarrow(f, g)$ is an amelioration, then the induced base change map $\zeta_{k}(\boldsymbol{\tau})=\mathbf{t}$ implies [Lic00, Lemma 5.10] the equation (between measures outside the set of critical values)

$$
\mathbf{F}_{W}\left(\zeta_{k}(\boldsymbol{\tau})\right)\left|\frac{\partial\left(t_{1}, t_{2}\right)}{\partial\left(\tau_{1}, \tau_{2}\right)}\right||d \boldsymbol{\tau}|=\mathcal{F}_{W, k}(\boldsymbol{\tau})|d \boldsymbol{\tau}|,
$$




\section{B. LICHTIN}

and $\mathcal{F}_{W, k}(\boldsymbol{\tau})$ is a finite sum of asymptotic monomials in $\boldsymbol{\tau}, \boldsymbol{\tau}$ (= a regular value) $\left.\in \operatorname{Im}\left(F_{k}, G_{k}\right)\right|_{W}$. The finite set of $\boldsymbol{\omega}$ that parametrize the asymptotic monomials for either $\mathcal{F}_{W, k}(\boldsymbol{\tau})$ will also be denoted by $\mathcal{Y}_{W}$.

A property that is needed in $\S \S 2$ and 3 is the absolute integrability of any asymptotic monomial. The notation is as above and we introduce $\boldsymbol{t}$ to denote either $\mathbf{t}$ or $\boldsymbol{\tau}$.

Lemma 1.10. For any good $\mathbf{P}$ wedge $W$ and $\boldsymbol{\omega}=(\iota, \mathbf{M}, \mathbf{b}, \boldsymbol{\chi}) \in \mathcal{Y}_{W}$, one has $\int_{C_{\llcorner}^{*}}\left|\mathcal{M}_{\boldsymbol{\omega}}\right||d \boldsymbol{t}|<\infty$.

Proof. By reindexing, one may assume $\iota=\{1,2\}$. Via the cone $\mathcal{C}_{\iota}=\left\langle\left(N_{1}, M_{1}\right),\left(N_{2}, M_{2}\right)\right\rangle$, introduce the monomial transformation

$$
\Theta: \mathbf{T} \underset{\text { def }}{=}\left(T_{1}, T_{2}\right) \rightarrow \boldsymbol{t}=\left(T_{1}^{N_{1}} T_{2}^{M_{1}}, T_{1}^{N_{2}} T_{2}^{M_{2}}\right) .
$$

If $B>0$, then $\mathbf{M}+\left\{\operatorname{ord} \Theta(\mathbf{T}): 0 \leqslant \operatorname{ord} T_{i} \leqslant B, i=1,2\right\}$ is a finite subset of $\mathbf{M}+\mathcal{C}_{\iota}$. Next, rewrite the integrand in terms of $\mathbf{T}$, and restrict ord $\mathbf{T}$ to $[0, B]^{2}$. It now suffices to bound each $\log ^{b_{i}}\left|T_{1}^{N_{i}} T_{2}^{M_{i}}\right|$ factor by $c_{\varepsilon}\left|T_{1}^{N_{i}} T_{2}^{M_{i}}\right|^{-\varepsilon}$ and show that if $\varepsilon \ll 1$, then

$$
\lim _{B \rightarrow \infty} \int_{\left\{\mathbf{T}: \operatorname{ord} \mathbf{T} \in[0, B]^{2}\right\}}\left|T_{1}\right|^{-\left(N_{1} v_{1}+M_{1} v_{2}+1\right)-\varepsilon}\left|T_{2}\right|^{-\left(N_{2} v_{1}+M_{2} v_{2}+1\right)-\varepsilon}|d \mathbf{T}|<\infty .
$$

Since $v \in \mathcal{L}_{1} \cap \mathcal{L}_{2}$, it follows that $N_{i} v_{1}+M_{i} v_{2}=-\mu_{i}<0$ for each $i$. Thus, the exponent of each $\left|T_{i}\right|$ is strictly larger than -1 , provided that $\varepsilon$ is sufficiently small. So, the limit as $B \rightarrow+\infty$ exists and is finite. This completes the proof of the lemma.

\subsection{The Fourier transform of the fiber integral}

Fix an additive character $\Psi$ of $\mathbb{Q}_{p}$ that equals one on $\mathbb{Z}_{p}$ and is nontrivial on $(p)^{-1} \mathbb{Z}_{p}$. The Fourier transform at $\boldsymbol{\lambda}=\left(\lambda_{1}, \lambda_{2}\right)$ of the fiber integral $F(t)$ equals

$$
F^{*}(\boldsymbol{\lambda})=\int F(t) \Psi(\boldsymbol{\lambda} \cdot t)|d t|
$$

A simple calculation shows that $F^{*}\left(\mathbf{a} / p^{r}\right)=G\left(\mathbf{a} / p^{r}\right)$.

Given a good $\mathbf{P}$ wedge $W=\theta U(\boldsymbol{x})$, so that $\theta(\boldsymbol{x})=\mathbf{x}$, the Fourier transform of the local contribution $F_{W}(t)$ is

$$
\begin{aligned}
F_{W}^{*}(\boldsymbol{\lambda}) & \underset{\text { def }}{=} \int F_{W}(t) \Psi(\boldsymbol{\lambda} \cdot t)|d t| \\
& =\Psi(\boldsymbol{\lambda} \cdot \mathbf{P}(\mathbf{x})) \int \mathbf{F}_{W}(\mathbf{t}) \Psi(\boldsymbol{\lambda} \cdot \mathbf{t})|d \mathbf{t}| \underset{\text { def }}{=} G_{W}(\boldsymbol{\lambda}) .
\end{aligned}
$$

Remark. By (1.4) it follows that $G(\boldsymbol{\lambda})=\sum_{i} G_{W_{i}}(\boldsymbol{\lambda})$. To prove Theorem A, it therefore suffices to show that for any good $\mathbf{P}$ wedge $W$, there exists $\alpha=\alpha(W)<0$ such that $G_{W}(\boldsymbol{\lambda})=O_{\varepsilon}\left(|\boldsymbol{\lambda}|^{\alpha+\varepsilon}\right)$, where $|\boldsymbol{\lambda}|=\max \left\{\left|\lambda_{1}\right|,\left|\lambda_{2}\right|\right\}$.

If $\boldsymbol{x}$ is a good point for $(f, g)$, then Remark 1.9 implies that

$$
\mathbf{F}_{W}^{*}(\boldsymbol{\lambda})=\sum_{\boldsymbol{\omega} \in \mathcal{Y}_{W}} c_{\boldsymbol{\omega}} \cdot \mathcal{M}_{\boldsymbol{\omega}}^{*}(\boldsymbol{\lambda}), \quad(\boldsymbol{\omega}=(\iota, \mathbf{M}, \mathbf{b}, \boldsymbol{\chi}))
$$

where

$$
\begin{aligned}
\mathcal{M}_{\omega}^{*}(\boldsymbol{\lambda}) & \underset{\operatorname{def}}{=} \int_{\left.\operatorname{Im}(f, g)\right|_{W}} \mathcal{M}_{\boldsymbol{\omega}}(\mathbf{t}) \Psi(\boldsymbol{\lambda} \cdot \mathbf{t})|d \mathbf{t}| \\
& =\int_{\left.\operatorname{Im}(f, g)\right|_{W}} \chi_{1}\left(\operatorname{act} t_{1}\right) \chi_{2}\left(\operatorname{act} t_{2}\right)\left|t_{1}\right|^{-v_{1}-1}\left|t_{2}\right|^{-v_{2}-1} \log ^{b_{1}}\left|t_{1}\right| \log ^{b_{2}}\left|t_{2}\right| \Psi(\boldsymbol{\lambda} \cdot \mathbf{t})|d \mathbf{t}| .
\end{aligned}
$$


In this case, the decay for $G_{W}(\boldsymbol{\lambda})$ follows from the existence, for each $\boldsymbol{\omega} \in \mathcal{Y}_{W}$, of $\alpha(\boldsymbol{\omega})<0$ such that

$$
\mathcal{M}_{\omega}^{*}(\boldsymbol{\lambda})=O_{\varepsilon}\left(|\boldsymbol{\lambda}|^{\alpha(\boldsymbol{\omega})+\varepsilon}\right)
$$

It would evidently suffice to set $\alpha(W)=\max _{\boldsymbol{\omega} \in \mathcal{Y}_{W}} \alpha(\boldsymbol{\omega})$.

If $\boldsymbol{x}$ is a bad point for $(f, g), \zeta_{k}:\left(F_{k}, G_{k}\right) \rightarrow(f, g)$ is a type $k$ amelioration and $\mathcal{M}_{\boldsymbol{\omega}}, \boldsymbol{\omega} \in \mathcal{Y}_{W}$, is an asymptotic monomial for the fiber integral $\mathcal{F}_{W, k}(\boldsymbol{\tau})$, then Remark 1.9 again implies

$$
\begin{aligned}
\int \mathbf{F}_{W}\left(\zeta_{k}(\boldsymbol{\tau})\right) \Psi\left(\boldsymbol{\lambda} \cdot \zeta_{k}(\boldsymbol{\tau})\right)\left|\frac{\partial\left(t_{1}, t_{2}\right)}{\partial\left(\tau_{1}, \tau_{2}\right)}\right||d \boldsymbol{\tau}| & =\int \mathcal{F}_{W, k}(\boldsymbol{\tau}) \Psi\left(\boldsymbol{\lambda} \cdot \zeta_{k}(\boldsymbol{\tau})\right)|d \boldsymbol{\tau}| \\
& =\sum_{\boldsymbol{\omega}} c_{\boldsymbol{\omega}} \cdot \int_{\left.\operatorname{Im}\left(F_{k}, G_{k}\right)\right|_{W}} \mathcal{M}_{\boldsymbol{\omega}}(\boldsymbol{\tau}) \Psi\left(\boldsymbol{\lambda} \cdot \zeta_{k}(\boldsymbol{\tau})\right)|d \boldsymbol{\tau}| .
\end{aligned}
$$

Thus, the decay for $G_{W}(\boldsymbol{\lambda})$ would follow in the same way by proving, for each $k$, the existence of $\alpha_{k}(\boldsymbol{\omega})<0$ such that

$$
\int \mathcal{M}_{\boldsymbol{\omega}}(\boldsymbol{\tau}) \Psi\left(\boldsymbol{\lambda} \cdot \zeta_{k}(\boldsymbol{\tau})\right)|d \boldsymbol{\tau}|=O_{\varepsilon}\left(\left|\lambda_{k}\right|^{\alpha_{k}(\boldsymbol{\omega})+\varepsilon}\right) \quad \text { in the cone }|\boldsymbol{\lambda}|=\left|\lambda_{k}\right|
$$

Note that the left-hand side is not the Fourier transform of $\mathcal{M}_{\boldsymbol{\omega}}(\boldsymbol{\tau})$. Section 3 proves these local decay estimates and characterizes the exponent $\alpha(\boldsymbol{\omega})$ (respectively $\alpha_{k}(\boldsymbol{\omega})$ ) in terms of the axis intercepts of the polygon $\Gamma_{\iota}\left(\right.$ respectively $\left.\Gamma_{\iota, k}\right)$.

\section{The inner integral}

For fixed good $\mathbf{P}$ wedge $W$ and $\boldsymbol{\omega}=(\iota, \mathbf{M}, \mathbf{b}, \boldsymbol{\chi}) \in \mathcal{Y}_{W}$, the first step in bounding the double integrals in (1.8), (1.9) is to deduce a bound for an inner integral. Since Lemma 1.10 ensures that these integrals are independent of the order of iteration, one chooses, without loss of generality, to slice the domain of integration vertically. Writing $\boldsymbol{t}=(x, y)$ to denote $\mathbf{t}$ in (1.8) and $\boldsymbol{\tau}$ in (1.9), for fixed $x$, the inner integral becomes an oscillatory integral over the vertical slice at $x$. By definition, this is equal to the set of $y$ such that ord $\boldsymbol{t}=(\operatorname{ord} x, \operatorname{ord} y) \in \mathbf{M}+\mathcal{C}_{\iota}^{*}$. It is then important to describe the structure of the vertical slices, as well as any variation in this structure, as ord $x$ varies.

An important feature of the $p$-adic integration is that there is an 'effective' subset of each vertical slice (see Definition 2.2), characterized by the property that over its complement the oscillating factor forces the integral to vanish. Moreover, this property only depends on a simple inequality satisfied by ord $y$ and $\operatorname{ord} \lambda={ }_{\text {def }} \min \left\{\operatorname{ord} \lambda_{1}\right.$, ord $\left.\lambda_{2}\right\}$. The problem then is to parametrize this 'effective slice' of ord $y$ for fixed ord $x$. Lemma 2.3 shows that this set must be a segment (finite or infinite) of an arithmetic progression. Since the endpoints of the segment vary as ord $x$ varies, this variation must also be controlled.

One next shows in Lemma 2.5 that the set of ord $x$ can be partitioned into finitely many arithmetic progressions $\mathcal{S}_{1}, \ldots, \mathcal{S}_{K}$ so that if ord $x$ belongs to any one $\mathcal{S}_{i}$, then the endpoints of the effective slice can be simply expressed in terms of ord $x$, ord $\lambda$, and $i$. This suffices for bounding the inner integral over the slice at $x$. Although the elements of any progression will depend on ord $\lambda$, the number $K$ of distinct progressions is bounded uniformly of ord $\lambda$. The fact that the effective part of $\mathbf{M}+\mathcal{C}_{\iota}^{*}$ (the union of its effective vertical slices) is a finite union of 'fibered arithmetic progressions', the number of which is bounded uniformly in ord $\lambda$, is the main observation of this section, and is essential for the proof of Theorem 1 in $\S 3$.

One chooses $\mathcal{C}_{\iota}^{*}=\mathcal{C}_{\iota}$ throughout since this is the more difficult of the two possibilities. One writes $\mathbf{M}=\left(m_{1}, m_{2}\right), \operatorname{ord} \boldsymbol{t}={ }_{\text {def }}(\operatorname{ord} x, \operatorname{ord} y)=\left(r_{1}, r_{2}\right)$. The indices $\iota, \boldsymbol{\omega}$ are dropped from the notation since they are fixed throughout. By permuting coordinates one may assume $\iota=\{1,2\}$ and $D={ }_{\text {def }} N_{1} M_{2}-N_{2} M_{1}>0$. 


\section{B. LICHTIN}

\subsection{Parametrization of the lattice points ord $t$}

Given a parametrization $W=\theta U(\boldsymbol{x})$ of the good $\mathbf{P}$ wedge, for which the matrix of multiplicities $\mathbf{A}(\boldsymbol{x})$ is given in Definition 1.7, one may assume that $\boldsymbol{t}$ belongs to the image of the monomial map

$$
\boldsymbol{m}: \mathbf{z} \in U(\boldsymbol{x})=\prod_{i}\left\{z_{i}: \text { ord } z_{i} \geqslant e\right\} \rightarrow\left(\mathbf{z}^{\mathbf{N}}, \mathbf{z}^{\mathbf{M}}\right),
$$

where $e$ is some nonnegative integer, and $\mathbf{N}=\left(N_{1}, \ldots, N_{R}\right), \mathbf{M}=\left(M_{1}, \ldots, M_{R}\right)$. The set ord $\boldsymbol{t}$ then equals

$$
\begin{aligned}
& \left\{\left(r_{1}, r_{2}\right): \text { there exists } \mathbf{e} \in[e, \infty)^{R} \cap \mathbb{Z}^{R} \text { and } \mathbf{k} \in \mathbb{Z}_{+}^{2}\right. \text { such that } \\
& \left.\qquad\left(\begin{array}{l}
r_{1} \\
r_{2}
\end{array}\right)=\left(\begin{array}{llll}
N_{1} & N_{2} & \cdots & N_{R} \\
M_{1} & M_{2} & \cdots & M_{R}
\end{array}\right) \mathbf{e}=\mathbf{M}+\left(\begin{array}{ll}
N_{1} & N_{2} \\
M_{1} & M_{2}
\end{array}\right) \mathbf{k}\right\} .
\end{aligned}
$$

Set $\mathcal{K}$ to denote the set of $\mathbf{k} \in \mathbb{Z}_{+}^{2}$ for which (2.1) admits a solution (i.e. there exists an integral $\mathbf{e} \in[e, \infty)^{r}$ so that (2.1) holds). The structure of $\mathcal{K}$ is as follows.

Lemma 2.1. Assume $\mathcal{K} \neq \emptyset$. Then $\mathcal{K}=\bigcup_{u=1}^{U} \mathcal{K}_{u}$, with $U \leqslant D^{R}$, such that each $\mathcal{K}_{u}$ is a subset of $\mathbb{Z}_{+}^{2}$ of the form $\mathcal{H}_{u} \cap \mathbb{Z}_{+}^{2}$, where $\mathcal{H}_{u}$ is an unbounded subset of $[0, \infty)^{2}$. The boundary $\partial \mathcal{H}_{u}$ consists of exactly one vertical ray, one horizontal ray, and finitely many horizontal (respectively vertical) segments $h_{1}, \ldots, h_{i_{0}}$ (respectively $v_{1}, \ldots, v_{i_{0}}$ ), such that the path $h_{1} * v_{1} * h_{2} * v_{2} * \cdots * h_{i_{0}} * v_{i_{0}}$ forms a staircase descending from left to right (see Figure 2).

Proof. Given $\mathbf{e} \in[e, \infty)^{R} \cap \mathbb{Z}^{R}$, set $\mathbf{e}^{\prime}=\mathbf{e}-(e, \ldots, e)$ and

$$
\hat{\mathbf{M}}=\mathbf{M}-A(\boldsymbol{x})\left(\begin{array}{c}
e \\
\vdots \\
e
\end{array}\right)
$$

(recall that $A(\boldsymbol{x})$ denotes the first two rows of $\mathbf{A}(\boldsymbol{x})$ ). Multiplying the matrix equation in (2.1) on the left by $\left(\begin{array}{cc}M_{2} & -N_{2} \\ -M_{1} & N_{1}\end{array}\right)$, it follows that (2.1) holds if and only if

$$
D\left(\begin{array}{c}
e_{1}^{\prime} \\
e_{2}^{\prime}
\end{array}\right)+\mathcal{A} \mathbf{e}^{\prime \prime}=\mathbf{M}^{\prime}+D \mathbf{k}
$$

where $\mathbf{e}^{\prime \prime}=\left(e_{3}^{\prime}, \ldots, e_{R}^{\prime}\right), \mathbf{M}^{\prime} \in \mathbb{Z}^{2}$, and

$$
\mathcal{A}=\left(\begin{array}{lll}
a_{3} & \cdots & a_{R} \\
b_{3} & \cdots & b_{R}
\end{array}\right)=\left(\begin{array}{cc}
M_{2} & -N_{2} \\
-M_{1} & N_{1}
\end{array}\right)\left(\begin{array}{lll}
N_{3} & \cdots & N_{R} \\
M_{3} & \cdots & M_{R}
\end{array}\right) .
$$

This implies that (2.1) has a solution $\mathbf{e}^{\prime}, \mathbf{k}$ only if the congruence

$$
\mathcal{A} \mathbf{e}^{\prime \prime} \equiv \mathbf{M}^{\prime}(\bmod D)
$$

is satisfied. Set $\mathcal{Y}=\left\{\boldsymbol{\epsilon}^{\prime \prime} \in[0, D)^{R-2} \cap \mathbb{Z}^{R-2}: \boldsymbol{\epsilon}^{\prime \prime}\right.$ is a solution of $\left.(2.2)\right\}$. It is clear that $\# \mathcal{Y} \leqslant D^{R-2}$.

Assuming that $\mathcal{Y} \neq \emptyset$, set $\mathcal{Y}=\left\{\boldsymbol{\epsilon}_{j}^{\prime \prime}\right\}_{j=1}^{J}$, and choose any $\boldsymbol{\epsilon}_{j}^{\prime \prime}$. Viewing $\boldsymbol{\epsilon}_{j}^{\prime \prime}$ as an element of $(\mathbb{Z} / D)^{R-2}$, lift it to $\mathbb{Z}^{R-2}$ by introducing an integral vector $\mathbf{L}=\left(\ell_{3}, \ldots, \ell_{R}\right)$ and setting $\mathbf{e}^{\prime \prime}=$ $\boldsymbol{\epsilon}_{j}^{\prime \prime}+D \mathbf{L}$. Then $(2.1)$ has a solution e such that $\mathbf{e}^{\prime \prime} \equiv \boldsymbol{\epsilon}_{j}^{\prime \prime}(\bmod D)$ if and only if there exist integers $e_{1}^{\prime}, e_{2}^{\prime} \geqslant 0$ and $\mathbf{e}^{\prime \prime} \geqslant(0, \ldots, 0)$ such that

$$
\left(\begin{array}{l}
e_{1}^{\prime} \\
e_{2}^{\prime}
\end{array}\right)+\mathcal{A} \mathbf{L}=\mathbf{M}_{j}^{\prime \prime}+\left(\begin{array}{l}
k_{1} \\
k_{2}
\end{array}\right), \quad \text { where } \mathbf{M}_{j}^{\prime \prime}=\frac{1}{D}\left\{\mathbf{M}^{\prime}-\mathcal{A} \boldsymbol{\epsilon}_{j}^{\prime \prime}\right\} \underset{\text { def }}{=}\left(\mu_{1 j}, \mu_{2 j}\right) \in \mathbb{Z}^{2} .
$$


ON A QUESTION OF IGUSA, II

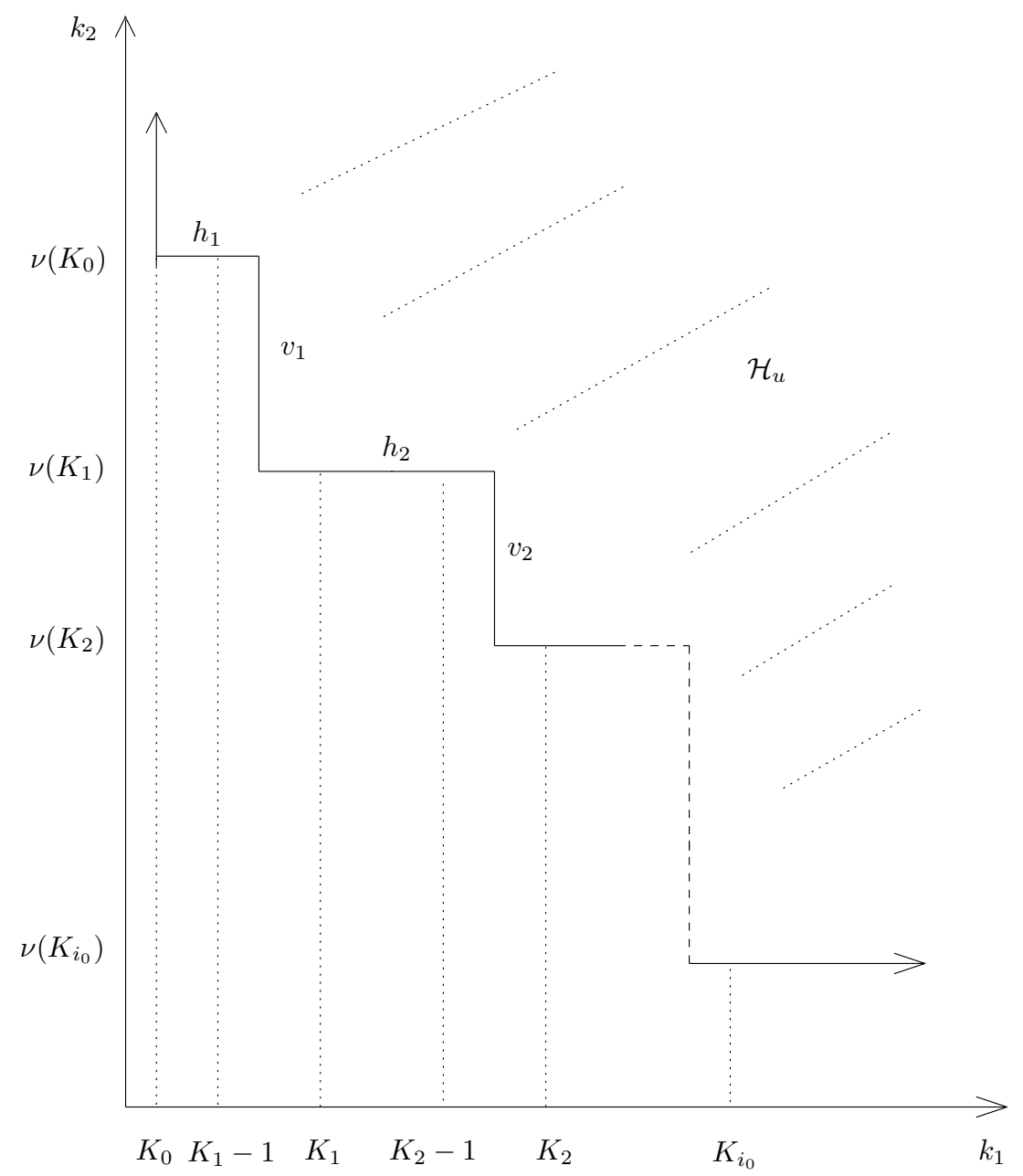

FiguRe 2.

Now, for each $j$ and $\mathbf{k} \in \mathbb{Z}_{+}^{2}$, set

$$
\mathcal{X}_{j}(\mathbf{k})=\left\{\mathbf{L} \in \mathbb{Z}_{+}^{R-2}: \sum_{i=3}^{R} a_{i} \ell_{i} \leqslant k_{1}+\mu_{1 j} \text { and } \sum_{i=3}^{R} b_{i} \ell_{i} \leqslant k_{2}+\mu_{2 j}\right\}
$$

and $\mathcal{K}_{j}=\left\{\mathbf{k}: \mathcal{X}_{j}(\mathbf{k}) \neq \emptyset\right\}$. It is then clear that $\mathcal{K}=\bigcup_{j=1}^{J} \mathcal{K}_{j}$.

To verify the asserted property of $\mathcal{K}_{j}$, set, for each $k_{1} \geqslant 0$,

$$
\nu_{j}\left(k_{1}\right)=\inf \left\{k_{2} \geqslant 0: \mathcal{X}_{j}\left(k_{1}, k_{2}\right) \neq \emptyset\right\}
$$

Observe that $\nu_{j}\left(k_{1}+1\right) \leqslant \nu_{j}\left(k_{1}\right)$ since $\mathcal{X}_{j}\left(k_{1}, k_{2}\right) \neq \emptyset$ implies $\mathcal{X}_{j}\left(k_{1}+1, k_{2}\right) \neq \emptyset$. Moreover, it is clear that for each $\left(k_{1}, \nu_{j}\left(k_{1}\right)\right)$ and any $k_{2} \geqslant \nu_{j}\left(k_{1}\right)$, there exist $e_{1}^{\prime}, e_{2}^{\prime} \geqslant 0$ and $\mathbf{L} \in \mathbb{Z}_{+}^{R-2}$ such that

$$
\left(\begin{array}{l}
e_{1}^{\prime} \\
e_{2}^{\prime}
\end{array}\right)+\mathcal{A} \mathbf{L}=\mathbf{M}_{j}^{\prime \prime}+\left(\begin{array}{l}
k_{1} \\
k_{2}
\end{array}\right)
$$


B. LICHTIN

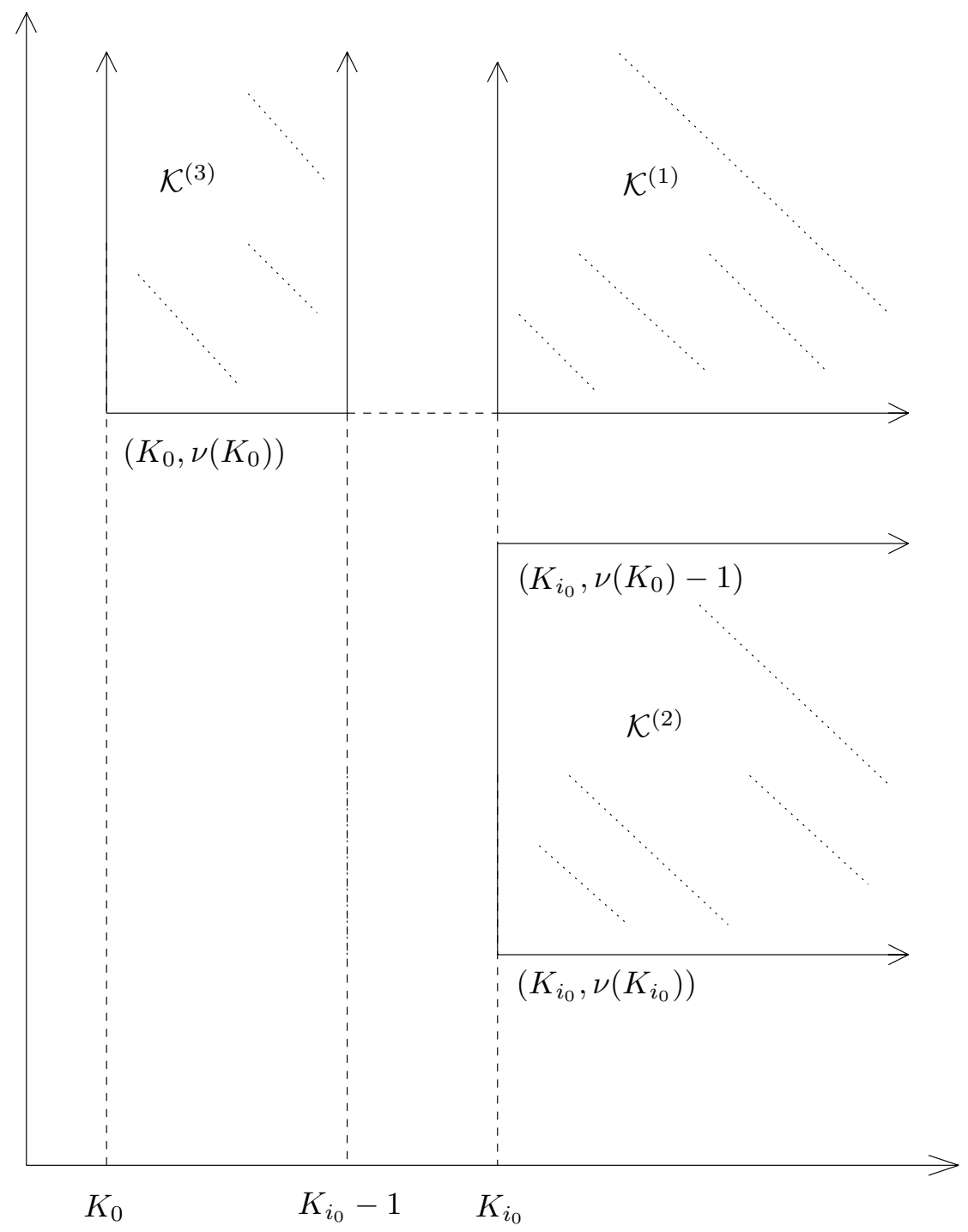

FIGURE 3.

Define the following indices:

$$
\begin{aligned}
& K_{0}=\inf \left\{k_{1}: \mathcal{X}_{j}\left(k_{1}, k_{2}\right) \neq \emptyset \text { for some } k_{2} \geqslant 0\right\} \\
& K_{1}=\inf \left\{k_{1}>K_{0}: \nu_{j}\left(k_{1}\right)<\nu_{j}\left(K_{0}\right)\right\} \\
& K_{i}=\inf \left\{k_{1}>K_{i-1}: \nu_{j}\left(k_{1}\right)<\nu_{j}\left(K_{i-1}\right)\right\} \quad \text { for each } i \geqslant 2 .
\end{aligned}
$$

Since $\nu_{j}\left(K_{0}\right)>\nu_{j}\left(K_{1}\right)>\nu_{j}\left(K_{2}\right)>\cdots \geqslant 0$, there exists a smallest index $i_{0} \geqslant 0$ such that $k_{1}>K_{i_{0}}$ implies $\nu_{j}\left(k_{1}\right)=\nu_{j}\left(K_{i_{0}}\right)$.

One can then connect the ascending vertical ray starting at $\left(K_{0}, \nu_{j}\left(K_{0}\right)\right)$ to the horizontal ray to the right of $\left(K_{i_{0}}, \nu_{j}\left(K_{i_{0}}\right)\right)$ by means of a finite number of horizontal followed by vertical segments that contain the points $\left(K_{i}, \nu_{j}\left(K_{i}\right)\right), 0 \leqslant i \leqslant i_{0}$ (see Figure 2 ). The region $\mathcal{H}_{j}$ whose boundary is such a path satisfies all the properties of the lemma.

Note. In the following discussion, a given $j \in[1, J]$ will be chosen and fixed throughout. For simplicity, it will then be dropped as a subscript for $\mathcal{K}$. 


\section{On A QUESTION OF Igusa, II}

It follows that if $i_{0}>0$, then there exists at most a finite subset $\mathcal{K}^{\prime}$ such that $\mathcal{K}^{*}={ }_{\text {def }} \mathcal{K}-\mathcal{K}^{\prime}=$ $\bigcup_{i=1}^{3} \mathcal{K}^{(i)}$, where (see Figure 3 ):

(a) $\mathbf{k} \in \mathcal{K}^{(1)}$ if and only if there exist $e_{1}, f_{1} \in \mathbb{Z}_{+}$such that $k_{1} \geqslant e_{1}$ and $k_{2} \geqslant f_{1}$;

(b) $\mathbf{k} \in \mathcal{K}^{(2)}$ if and only if there exist $e_{2}, f_{2}, g_{2} \in \mathbb{Z}_{+}$such that $k_{1} \geqslant e_{2}$ and $f_{2} \leqslant k_{2} \leqslant g_{2}$;

(c) $\mathbf{k} \in \mathcal{K}^{(3)}$ if and only if there exist $e_{3}, f_{3}, g_{3} \in \mathbb{Z}_{+}$such that $e_{3} \leqslant k_{1} \leqslant f_{3}$ and $k_{2} \geqslant g_{3}$.

In particular, using the notation in Figure 2, one can choose

$\left(e_{1}, f_{1}\right)=\left(K_{i_{0}}, \nu\left(K_{0}\right)\right) ; \quad\left(e_{2}, f_{2}, g_{2}\right)=\left(K_{i_{0}}, \nu\left(K_{i_{0}}\right), \nu\left(K_{0}\right)-1\right) ; \quad\left(e_{3}, f_{3}, g_{3}\right)=\left(K_{0}, K_{i_{0}}-1, \nu\left(K_{0}\right)\right)$.

When $i_{0}=0$, one writes $\mathcal{K}^{*}=\mathcal{K}^{(1)}$ to be consistent with (2.1). Additional precision will not be needed since such bounds are independent of the parameters $\lambda_{1}, \lambda_{2}$.

Define the corresponding sets for each $i$ :

$$
\mathcal{C}^{(i)}=\left\{k_{1}\left(N_{1}, M_{1}\right)+k_{2}\left(N_{2}, M_{2}\right)+\mathbf{M}: \mathbf{k} \in \mathcal{K}^{(i)}\right\}, \quad C^{(i)}=\left\{\boldsymbol{t}: \text { ord } \boldsymbol{t} \in \mathcal{C}^{(i)}\right\} .
$$

Since $\mathcal{C}^{*}=\mathcal{C}-\bigcup_{i=1}^{3} \mathcal{C}^{(i)}$ is a finite set, the contribution to the integrals (1.8), (1.9) over those $\boldsymbol{t}$ with ord $\boldsymbol{t} \in \mathcal{C}^{*}$ is zero for $|\boldsymbol{\lambda}| \gg 1$. This justifies the subsequent attention paid solely to $\mathcal{C}^{(1)} \cup \mathcal{C}^{(2)} \cup \mathcal{C}^{(3)}$. Intuitively, $\mathcal{C}^{(2)}$ (respectively $\mathcal{C}^{(3)}$ ) can be viewed as containing the points in $\mathcal{C}$ that cluster asymptotically at infinity along its lower (respectively upper) boundary ray.

Definition 2.2. As noted in the introduction to $\S 2$, the choice made in this article is to integrate first with respect to $y$, which means that each $\mathcal{C}^{(i)}$ is to be thought of as the union of its 'vertical' slices, defined for a fixed $r_{1}$, and $x$ ( ord $x=r_{1}$ ) as follows. Recalling the monomial map $\boldsymbol{m}$ defined at the beginning of $\S 2.1$, set

$$
\begin{gathered}
\mathcal{V}^{(i)}\left(r_{1}\right)=\left\{r_{2}:\left(r_{1}, r_{2}\right) \in \mathcal{C}^{(i)}\right\}, \\
V^{(i)}(x)=\left\{y:(x, y) \in \operatorname{Im}(\boldsymbol{m}) \text { and ord } y \in \mathcal{V}^{(i)}\left(r_{1}\right)\right\} .
\end{gathered}
$$

Application to the proof of (1.9) when $k=1$ means that one sets $y=\tau_{2}$, the coordinate in the range of $G_{1}$, and bounds

$$
\int_{V^{(i)}(x)}|y|^{-v_{2}-1} \log ^{b_{2}}|y| \chi_{2}(\operatorname{ac} y) \Psi\left(\lambda_{1} y\right)|d y| .
$$

Application to the proof of (1.8) (respectively (1.9)) with $k=2$ means that one sets $y=t_{2}$, the coordinate in the range of $g$ (respectively $y=\tau_{2}$, the coordinate in the range of $G_{2}$ ), and bounds

$$
\int_{V^{(i)}(x)}|y|^{-v_{2}-1} \log ^{b_{2}}|y| \chi_{2}(\operatorname{ac} y) \Psi\left(\lambda_{2} y\right)|d y| .
$$

Choosing $\lambda=\lambda_{1}$ for (2.3) (respectively $\lambda_{2}$ for (2.4)), a feature specific to the $p$-adic context is that there is an 'effective' subset $E^{(i)}(x, \lambda)$ of each $V^{(i)}(x)$, characterized by the property that for each $\chi_{2}$

$$
0=\int_{V^{(i)}(x)-E^{(i)}(x, \lambda)}|y|^{-v_{2}-1} \log ^{b_{2}}|y| \chi_{2}(\operatorname{ac} y) \Psi(\lambda y)|d y| .
$$

To see this it is first necessary to describe more precisely the set of pairs (ac $x$, ac $y$ ) when $(x, y) \in \operatorname{Im}(\boldsymbol{m})$. Given the $2 \times 2$ determinant $D$ from above, set $\delta\left(=\delta_{\iota}\right)=\operatorname{ord} D$.

Claim 1. There exist $\mathbf{b}_{1}, \ldots, \mathbf{b}_{T} \in \mathcal{U}_{p}^{2} \bmod p^{2 \delta+1}$ such that

$$
\{(\operatorname{ac} x, \operatorname{ac} y):(x, y) \in \operatorname{Im}(\boldsymbol{m})\}=\bigsqcup_{j=1}^{T}\left[\mathbf{b}_{j}\right]_{2 \delta+1} \quad \text { (a disjoint union). }
$$




\section{B. LICHTIN}

Proof. Express $\mathcal{U}_{p}^{n}$ as a disjoint union of cosets $\bmod p^{\delta+1}, \mathcal{U}_{p}^{n}=\bigcup_{j=1}^{J}\left[\boldsymbol{u}_{j}\right]_{\delta+1}$. Define the vectors $\mathbf{b}_{1}, \ldots, \mathbf{b}_{T} \in \mathcal{U}_{p}^{2}$ to be the distinct elements of the set $\left\{\boldsymbol{m}\left(\boldsymbol{u}_{j}\right)\right\}_{1}^{J}$. If $\mathbf{b}=\boldsymbol{m}(\boldsymbol{u})$ for some $\boldsymbol{u} \in\left\{\boldsymbol{u}_{j}\right\}_{1}^{J}$, it then suffices to show

$$
[\mathbf{b}]_{2 \delta+1}=\boldsymbol{m}\left([\boldsymbol{u}]_{\delta+1}\right) .
$$

A standard lifting argument shows (2.6). The first step of the induction argument is as follows. Write $\boldsymbol{u} \equiv \boldsymbol{\xi}_{0}\left(\bmod p^{\delta+1}\right)$ and $\mathbf{b} \equiv \boldsymbol{\beta}_{0}\left(\bmod p^{2 \delta+1}\right)$. Given any $\boldsymbol{\beta}_{1} \in\{0,1, \ldots, p-1\}^{2}$ one shows the existence of $\boldsymbol{\xi}_{1} \in\{0,1, \ldots, p-1\}^{n}$ such that

$$
\boldsymbol{m}\left(\boldsymbol{\xi}_{0}+p^{\delta+1} \boldsymbol{\xi}_{1}\right) \equiv \boldsymbol{\beta}_{0}+p^{2 \delta+1} \boldsymbol{\beta}_{1}\left(\bmod p^{2 \delta+2}\right) .
$$

Via Taylor's formula,

$$
\begin{aligned}
\boldsymbol{m}\left(\boldsymbol{\xi}_{0}+p^{\delta+1} \boldsymbol{\xi}_{1}\right) & =\boldsymbol{m}\left(\boldsymbol{\xi}_{0}\right)+p^{\delta+1} d \boldsymbol{m}\left(\boldsymbol{\xi}_{0}\right)\left(\boldsymbol{\xi}_{1}\right) \quad\left(\bmod p^{2 \delta+2}\right) \\
& =\boldsymbol{\beta}_{0}+p^{2 \delta+1} \gamma\left(\boldsymbol{\xi}_{0}\right)+p^{\delta+1} d \boldsymbol{m}\left(\boldsymbol{\xi}_{0}\right)\left(\boldsymbol{\xi}_{1}\right) \quad\left(\bmod p^{2 \delta+2}\right),
\end{aligned}
$$

where $\boldsymbol{\gamma}\left(\boldsymbol{\xi}_{0}\right)$ is some element of $\mathbb{Z}_{p}^{2}$. Thus, setting $p^{2 \delta+1}\left(\boldsymbol{\beta}_{1}-\boldsymbol{\gamma}\left(\boldsymbol{\xi}_{0}\right)\right)=p^{2 \delta+1} \boldsymbol{\zeta}_{0}+p^{2 \delta+2} \boldsymbol{\zeta}_{1}+\cdots$, it follows that the congruence $p^{\delta+1} d \boldsymbol{m}\left(\boldsymbol{\xi}_{0}\right)\left(\boldsymbol{\xi}_{1}\right) \equiv p^{2 \delta+1} \boldsymbol{\zeta}_{0}\left(\bmod p^{2 \delta+2}\right)$ is solvable if and only if $d \boldsymbol{m}\left(\boldsymbol{\xi}_{0}\right)\left(\boldsymbol{\xi}_{1}\right) \equiv p^{\delta} \boldsymbol{\zeta}_{0}\left(\bmod p^{\delta+1}\right)$ is solvable. Since $\boldsymbol{\xi}_{0} \neq \mathbf{0} \in \mathcal{U}_{p}^{n}$, the definition of $D, \delta$ implies that the determinant of the left-most $2 \times 2$ submatrix $\left(\begin{array}{cc}A_{1} & B_{1} \\ A_{2} & B_{2}\end{array}\right)$ of $d \boldsymbol{m}\left(\boldsymbol{\xi}_{0}\right)$ equals $p^{\delta} \cdot \mu$ for some $\mu \in \mathcal{U}_{p}$. A solution of the latter congruence is then given as $\boldsymbol{\xi}_{1}=\left(\xi_{11}, \xi_{21}, 0, \ldots, 0\right)$ where $\boldsymbol{\zeta}_{0}=\left(\zeta_{10}, \zeta_{20}\right)$ and

$$
\left(\xi_{11}, \xi_{21}\right) \equiv \frac{1}{p^{\delta} \cdot \mu} \cdot\left(\left|\begin{array}{cc}
p^{\delta} \zeta_{10} & B_{1} \\
p^{\delta} \zeta_{20} & B_{2}
\end{array}\right|,\left|\begin{array}{ll}
A_{1} & p^{\delta} \zeta_{10} \\
A_{2} & p^{\delta} \zeta_{20}
\end{array}\right|\right) \quad(\bmod p)
$$

It follows that $\boldsymbol{m}\left(\boldsymbol{\xi}_{0}+p^{\delta+1} \boldsymbol{\xi}_{1}\right)$ solves (2.7), completing the first step of the lifting. The remainder of the argument is a straightforward extension of this procedure and is left to the reader.

Note that this union of cosets need not equal $\mathcal{U}_{p}^{2}$.

Setting, for each $j=1, \ldots, T, V^{(i)}\left(x, \mathbf{b}_{j}\right)=_{\operatorname{def}}\left\{y:(\operatorname{ac} x\right.$, ac $\left.y) \in\left[\mathbf{b}_{j}\right]_{2 \delta+1}\right\}$, Claim 1 evidently implies

$$
\int_{V^{(i)}(x)}|y|^{-v_{2}-1} \log ^{b_{2}}|y| \chi_{2}(\operatorname{ac} y) \Psi(\lambda y)|d y|=\sum_{j} \int_{V^{(i)}\left(x, \mathbf{b}_{j}\right)}|y|^{-v_{2}-1} \log ^{b_{2}}|y| \chi_{2}(\operatorname{ac} y) \Psi(\lambda y)|d y| \text {. }
$$

Set $e_{\chi_{2}}$ as the conductor of $\chi_{2}$, and $E_{\chi_{2}}=\max \left\{e_{\chi_{2}}, 2 \delta+1\right\}$. An elementary calculation (see Lemma A.3) then shows the following.

CLAim 2. If $e+\operatorname{ord} \lambda+E_{\chi_{2}} \leqslant-1$, then for any $b \in \mathcal{U}_{p}$

$$
\int_{\substack{\left\{y: \text { ord } y=e \\ \text { ac } y \in[b]_{\chi_{2}}\right\}}}|y|^{-v_{2}-1} \log ^{b_{2}}|y| \chi_{2}(\operatorname{ac} y) \Psi(\lambda y)|d y|=0 .
$$

If $e+\operatorname{ord} \lambda+E_{\chi_{2}} \geqslant 0$, then

$$
\int_{\substack{\left\{y: \operatorname{ord} y=e \\ \operatorname{ac} y \in[b] E_{\chi_{2}}\right\}}}|y|^{-v_{2}-1} \log ^{b_{2}}|y| \chi_{2}(\operatorname{ac} y) \Psi(\lambda y)|d y|=p^{e v_{2}-E_{\chi_{2}}}(-e)^{b_{2}} \chi_{2}(b) \Psi\left(b p^{e} \lambda\right) .
$$

It is clear that the only $b$ that are of interest in the following are congruent mod $p^{2 \delta+1}$ to the second component $b_{j, 2}$ of some $\mathbf{b}_{j}$ appearing in Claim 1 .

Since $\mathcal{Y}_{W}$ is a finite set, the set of possible $\chi_{2}$ that can be the second component of a character pair $\chi$ for some $\boldsymbol{\omega} \in \mathcal{Y}_{W}$ is also finite. As a result, one can define the following integer

$$
\vartheta_{W}=\max E_{\chi_{2}} .
$$


For each $i, r_{1}, \lambda$, set

$$
\mathcal{E}^{(i)}\left(r_{1}, \lambda\right)=\mathcal{V}^{(i)}\left(r_{1}\right) \cap\left[-\operatorname{ord} \lambda-\vartheta_{W}, \infty\right),
$$

and for any $x$ with ord $x=r_{1}$, define

$$
E^{(i)}(x, \lambda)=\left\{y \in V^{(i)}(x): \text { ord } y \in \mathcal{E}^{(i)}\left(r_{1}, \lambda\right)\right\}
$$

It is clear that $(2.5)$ is satisfied (for each $\chi_{2}$ ) with this choice for the effective slice.

The first problem is the parametrization of the integral points in each $\mathcal{E}^{(i)}\left(r_{1}, \lambda\right)$. As the proof of Lemma 2.3 will indicate, it is simpler to parametrize $\mathcal{E}^{(i)}\left(r_{1}, \lambda\right)$ if - ord $\lambda$ is allowed to be arbitrarily large since this assumption leads to fewer cases that must be considered and, thus, to an easier estimation of the double integrals.

With the choices specified above for $\lambda$, the assumption ' - ord $\lambda$ is unbounded' is satisfied when (2.4) is the inner integral for (1.8) and $\boldsymbol{\lambda}$ is confined to the cone $\Omega_{2}=\left\{\boldsymbol{\lambda}:|\boldsymbol{\lambda}|=\left|\lambda_{2}\right|\right\}$. However, this evidently fails when $\boldsymbol{\lambda}=\left(\lambda_{1}, \lambda_{2}\right)$ is confined to the cone $\Omega_{1}=\left\{\boldsymbol{\lambda}:|\boldsymbol{\lambda}|=\left|\lambda_{1}\right|\right\}$ where $-\operatorname{ord} \lambda_{1}$ is unbounded but $-\operatorname{ord} \lambda_{2}$ need not be. To deal with this case, however, is trivial. Evidently, one inverts the order of integration and takes the inner integral over the 'horizontal' slices of each $\mathcal{C}^{(i)}$.

To have a uniform discussion across all possible cases encountered in the proofs of (1.8) and (1.9), it is useful to maintain the vertical orientation of the slices. To this end, it suffices to define a new map by inverting the order of $f$ and $g$. For the same pair of indices $\iota$ as above, it is clear that $\left(M_{1}, N_{1}\right),\left(M_{2}, N_{2}\right)$ are the generators of the cone $\hat{\mathcal{C}}_{\iota}$, associated with $(g, f)$, and the horizontal slices of $\mathcal{C}_{\iota}$ are now the vertical slices of $\hat{\mathcal{C}}_{\iota}$. So, one sets $\lambda=\lambda_{1}, x=g, r_{1}=$ ord $g$, replaces $\chi_{2}$ by $\chi_{1}$ (in order to define the appropriate variant of $\vartheta_{W}$ ), and then applies the following discussion to parametrize the effective part of each vertical slice of $\hat{\mathcal{C}}_{\iota}$ in the cone $\Omega_{1}$. Section 2.2 then applies this to bound the inner integral of $\left.\mathcal{M}_{\boldsymbol{\omega}}^{*}(\boldsymbol{\lambda})\right|_{\Omega_{1}}$ by integrating first with respect to $y$ where $y=t_{1}(=f)$.

Notation. One sets $\epsilon=-\operatorname{ord} \lambda-\vartheta_{W}$. The parameter $\epsilon$ is always assumed to be unbounded. The validity of an assertion that is contingent on the property $\epsilon \gg 1$ means that the assertion is satisfied when $\epsilon>c$, where $c$ is a constant, independent of $r_{1}, r_{2}, \lambda_{1}, \lambda_{2}$ (but depending on finitely many other constants).

The first point is as follows.

Lemma 2.3. Set $\gamma=\operatorname{gcd}\left(N_{1}, N_{2}\right)$. If $\gamma \nmid r_{1}-m_{1}$, then $\mathcal{E}^{(i)}\left(r_{1}, \lambda\right)=\emptyset$. If $\gamma \mid r_{1}-m_{1}$, then for each $i=1,2,3$, there exist $A_{i}=A_{i}\left(r_{1}\right)$ and $B_{i}=B_{i}\left(r_{1}, \epsilon\right)$ such that if $\mathcal{E}^{(i)}\left(r_{1}, \lambda\right) \neq \emptyset$ then

$$
\mathcal{E}^{(i)}\left(r_{1}, \lambda\right)=\left\{m_{2}+r_{1}^{\prime} \eta-q D^{\prime}: A_{i} \leqslant q \leqslant B_{i}\right\},
$$

where $r_{1}^{\prime}=\left(r_{1}-m_{1}\right) / \gamma, D^{\prime}=D / \gamma, \eta$ is an appropriately defined constant, and either $A_{i}>-\infty$ (if $\left.N_{2}>0\right)$ or $A_{i} \geqslant-\infty$ (if $\left.N_{2}=0\right)$.

Proof. The $A_{i}, B_{i}$ will be determined by explicitly given piecewise linear functions of $r_{1}$, at most one of which also depends on $\epsilon$.

It is convenient to denote the slopes of the boundary of $\mathcal{C}$ as follows:

$$
\alpha=\frac{M_{1}}{N_{1}}, \quad \beta= \begin{cases}\frac{M_{2}}{N_{2}} & \text { if } N_{2}>0 \\ +\infty & \text { if } N_{2}=0 .\end{cases}
$$

For any $i,\left(r_{1}, r_{2}\right) \in \mathcal{C}^{(i)}$ implies

$$
\left(r_{1}, r_{2}\right)=k_{1}\left(N_{1}, M_{1}\right)+k_{2}\left(N_{2}, M_{2}\right)+\left(m_{1}, m_{2}\right), \text { for some } k_{1}, k_{2} \geqslant 0 \text {. }
$$




\section{B. LICHTIN}

Setting $N_{j}^{\prime}=N_{j} / \gamma, j=1,2$, it is clear that $\mathcal{E}^{(i)}\left(r_{1}, \lambda\right)=\emptyset$, for each $i$, if $\gamma \nmid r_{1}-m_{1}$. Assuming then that $\gamma \mid r_{1}-m_{1}$, it follows that there exists $q \in \mathbb{Z}$ such that

$$
\left(k_{1}, k_{2}\right)=r_{1}^{\prime}\left(\eta_{2}, \eta_{1}\right)+q\left(N_{2}^{\prime},-N_{1}^{\prime}\right)
$$

where $\eta_{1}, \eta_{2}$ are the unique numbers with smallest absolute value and opposite sign such that $\eta_{2} N_{1}^{\prime}+\eta_{1} N_{2}^{\prime}=1$, if $N_{2}>0$. The fact that $r_{2} \in \mathcal{E}^{(i)}\left(r_{1}, \lambda\right)$ also requires that $k_{1} M_{1}+k_{2} M_{2}+m_{2} \geqslant \epsilon$. Thus,

$$
r_{2}=r_{1}^{\prime} \eta-q D^{\prime}+m_{2}, \quad \text { where } \eta=\eta_{2} M_{1}+\eta_{1} M_{2} .
$$

So, an upper bound for $q$ will always be

$$
q \leqslant \frac{r_{1}^{\prime} \eta+m_{2}-\epsilon}{D^{\prime}} \underset{\text { def }}{=} Q\left(r_{1}, \epsilon\right) .
$$

Note that if $N_{2}=0$, then $N_{1} \mid r_{1}-m_{1}$ must hold for there to be any $\left(r_{1}, r_{2}\right) \in \mathbf{M}+\left\langle\left(N_{1}, M_{1}\right)\right.$, $\left.\left(0, M_{2}\right)\right\rangle_{\mathbb{N}}$. In this case, it follows that $\eta=M_{1}, D^{\prime}=M_{2}$, and the inequality that defines $\mathcal{E}^{(i)}\left(r_{1}, \lambda\right)$ becomes $r_{2}=r_{1}^{\prime} M_{1}-q M_{2}+m_{2} \geqslant \epsilon$.

Other possible bounds for $q$ come from the intervals that determine membership in the different $\mathcal{K}^{(i)}$. Working them out is an elementary exercise. One finds the following, using straightforward calculations that are best left to the reader.

$(i=1) \quad A_{1} \leqslant q \leqslant B_{1}$ where

$$
\begin{aligned}
& A_{1} \underset{\text { def }}{=} \begin{cases}\frac{e_{1}-r_{1}^{\prime} \eta_{2}}{N_{2}^{\prime}} & \text { if } N_{2}>0 \\
-\infty & \text { if } N_{2}=0\end{cases} \\
& B_{1} \underset{\text { def }}{=} \min \left\{\frac{r_{1}^{\prime} \eta_{1}-f_{1}}{N_{1}^{\prime}}, Q\left(r_{1}, \epsilon\right)\right\} \text { for any } N_{2} .
\end{aligned}
$$

Thus, there exist constants $\rho_{1}, \zeta_{1}$ such that if $\left[A_{1}, B_{1}\right] \neq \emptyset$ and $\epsilon \gg 1$, then

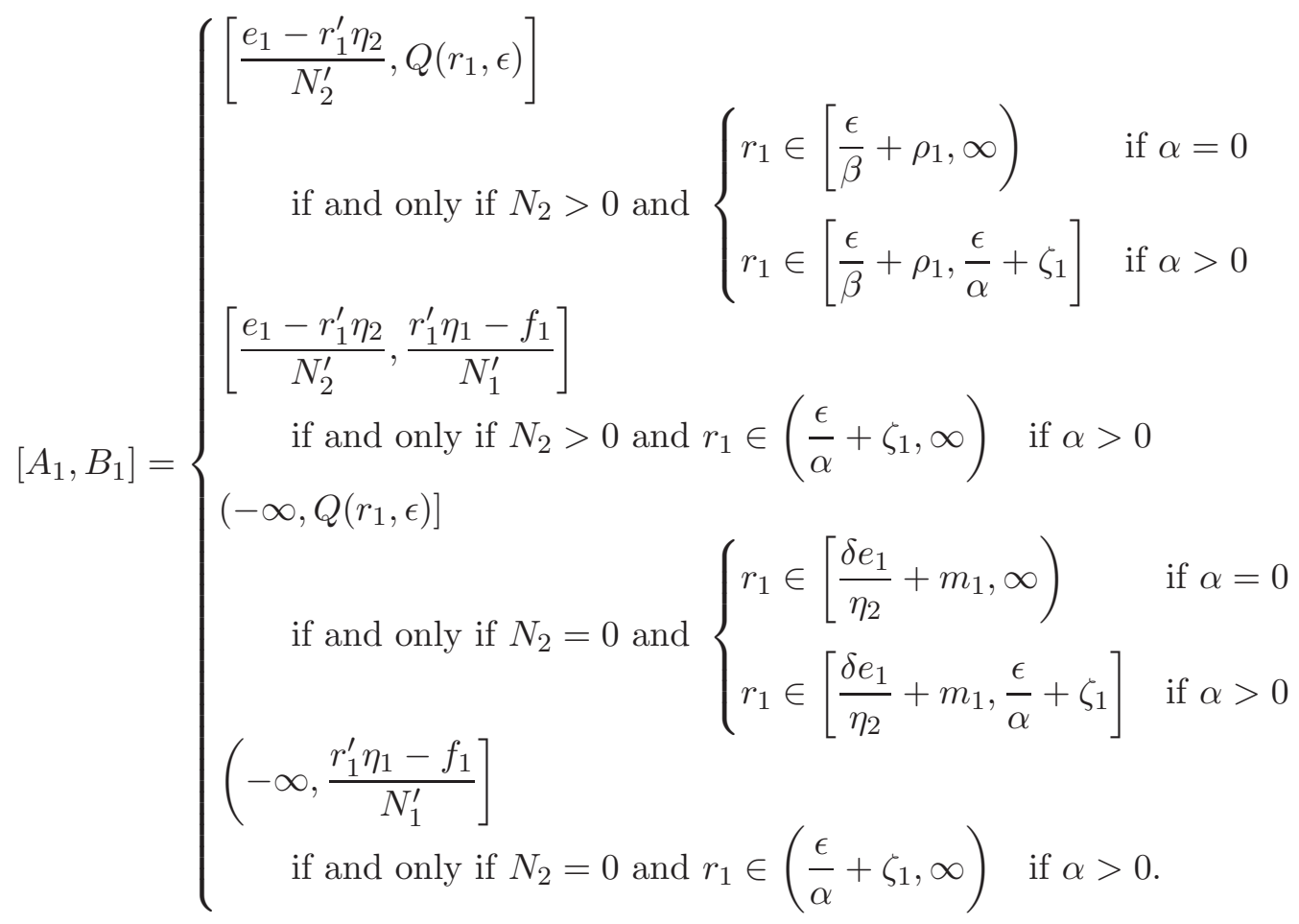




\section{On A QUESTION OF Igusa, II}

Note. An example of the simplification, afforded by the assumption $\epsilon \gg 1$ (see Definition 2.2), is the exclusion of the possibility that

$$
\left[A_{1}, B_{1}\right]=\left[\frac{e_{1}-r_{1}^{\prime} \eta_{2}}{N_{2}^{\prime}}, \frac{r_{1}^{\prime} \eta_{1}-f_{1}}{N_{1}^{\prime}}\right]
$$

when $\alpha=0$, since a simple check shows that this can only occur if $\epsilon \leqslant m_{2}+M_{2} f_{1}$.

$(i=2) \quad A_{2} \leqslant q \leqslant B_{2}$ where

$$
\begin{aligned}
& A_{2} \underset{\text { def }}{=} \begin{cases}\max \left\{\frac{e_{2}-r_{1}^{\prime} \eta_{2}}{N_{2}^{\prime}}, \frac{r_{1}^{\prime} \eta_{1}-g_{2}}{N_{1}^{\prime}}\right\} & \text { if } N_{2}>0 \\
\frac{r_{1}^{\prime} \eta_{1}-g_{2}}{N_{1}^{\prime}} & \text { if } N_{2}=0 ;\end{cases} \\
& B_{2} \underset{\text { def }}{=} \min \left\{\frac{r_{1}^{\prime} \eta_{1}-f_{2}}{N_{1}^{\prime}}, Q\left(r_{1}, \epsilon\right)\right\} \text { for any } N_{2} \text {. }
\end{aligned}
$$

Thus, there exist constants $\rho_{2}, \zeta_{2}$ such that if $\left[A_{2}, B_{2}\right] \neq \emptyset$ and $\epsilon \gg 1$, then $\alpha>0$ and

$$
\left[A_{2}, B_{2}\right]= \begin{cases}{\left[\frac{r_{1}^{\prime} \eta_{1}-g_{2}}{N_{1}^{\prime}}, Q\left(r_{1}, \epsilon\right)\right]} & \text { for } N_{2} \geqslant 0 \text { and } r_{1} \in\left[\frac{\epsilon}{\alpha}+\rho_{2}, \frac{\epsilon}{\alpha}+\zeta_{2}\right] \\ {\left[\frac{r_{1}^{\prime} \eta_{1}-g_{2}}{N_{1}^{\prime}}, \frac{r_{1}^{\prime} \eta_{1}-f_{2}}{N_{1}^{\prime}}\right]} & \text { for } N_{2} \geqslant 0 \text { and } r_{1} \in\left(\frac{\epsilon}{\alpha}+\zeta_{2}, \infty\right) .\end{cases}
$$

$(i=3) \quad A_{3} \leqslant q \leqslant B_{3}$ where

$$
\begin{aligned}
& A_{3}= \begin{cases}\frac{e_{3}-r_{1}^{\prime} \eta_{2}}{N_{2}^{\prime}} & \text { if } N_{2}>0 \\
-\infty & \text { if } N_{2}=0 ;\end{cases} \\
& B_{3}= \begin{cases}\min \left\{\frac{r_{1}^{\prime} \eta_{1}-g_{3}}{N_{1}^{\prime}}, \frac{f_{3}-r_{1}^{\prime} \eta_{2}}{N_{2}^{\prime}}, Q\left(r_{1}, \epsilon\right)\right\} & \text { if } N_{2}>0 \\
\min \left\{\frac{r_{1}^{\prime} \eta_{1}-g_{3}}{N_{1}^{\prime}}, Q\left(r_{1}, \epsilon\right)\right\} & \text { if } N_{2}=0 .\end{cases}
\end{aligned}
$$

Thus, there exist constants $\rho_{3}, \zeta_{3}$ such that if $\left[A_{3}, B_{3}\right] \neq \emptyset$ and $\epsilon \gg 1$, then

$$
\left[A_{3}, B_{3}\right]= \begin{cases}{\left[\frac{e_{3}-r_{1}^{\prime} \eta_{2}}{N_{2}^{\prime}}, Q\left(r_{1}, \epsilon\right)\right]} & \text { if and only if } N_{2}>0 \text { and } r_{1} \in\left[\frac{\epsilon}{\beta}+\rho_{3}, \frac{\epsilon}{\beta}+\zeta_{3}\right] \\ {\left[\frac{e_{3}-r_{1}^{\prime} \eta_{2}}{N_{2}^{\prime}}, \frac{f_{3}-r_{1}^{\prime} \eta_{2}}{N_{2}^{\prime}}\right]} & \text { if and only if } N_{2}>0 \text { and } r_{1} \in\left(\frac{\epsilon}{\beta}+\zeta_{3}, \infty\right) \\ \left(-\infty, Q\left(r_{1}, \epsilon\right)\right] & \text { if and only if } N_{2}=0 \text { and } r_{1} \in\left[\frac{e_{3}}{\eta_{2}}+m_{1}, \frac{f_{3}}{\eta_{2}}+m_{1}\right] .\end{cases}
$$

In addition, $N_{2}=0$ implies $\alpha>0$.

This completes the proof of the lemma.

Remarks.

(1) It is important to observe that the upper bound for $q$ is always $Q\left(r_{1}, \epsilon\right)$ whenever the lower bound for $r_{1}$ is a constant; that is, not a positive multiple of $\epsilon$. This is the source of nontrivial decay when no decay can be obtained from the integration in $x$ (see $\S 3$ ). 


\section{B. LICHTIN}

(2) Since $q$ is restricted to integral values, the end points of the intervals $\left[A_{i}, B_{i}\right]$ must be effectively replaced by $\left\lceil A_{i}\right\rceil=$ the smallest integer at least as large as $A_{i}$, and $B_{i}$ must be replaced by $\left[B_{i}\right]=$ the largest integer that is at most $B_{i}$. However, when one bounds the inner integral, it is also convenient, computationally, to replace the limits for $q$ by $A_{i}, B_{i}$. This introduces errors that depend on $\left\lceil A_{i}\right\rceil-A_{i}$ and $B_{i}-\left[B_{i}\right]$. Each of these are, of course, $O(1)$ in $r_{1}, \epsilon$. However, since the outer integral in $r_{1}$ must also be bounded, it could occur that the $O(1)$ errors accumulate and produce a poorer bound for the two-dimensional integral than would be acceptable. To eliminate this possibility, one needs to control the behavior uniformly in $r_{1}$.

To this end, it is natural to take each $A_{i}, B_{i}$ in (2.3) and specify the congruence class of its numerator modulo the corresponding denominator. This leads to a finite set of congruence classes for each $i=1,2,3$. For each possible congruence class, the errors $\left\lceil A_{i}\right\rceil-A_{i}$ and $B_{i}-\left[B_{i}\right]$ will then be constant. This constant appears explicitly in the estimates for the inner integral (see (2.9)(2.11)). It will also now be convenient to distinguish between the cases $\beta<\infty$ and $\beta=\infty$ since the expressions for $A_{i}, B_{i}$ depend on the finiteness of $\beta$.

As a result, the subsequent analysis splits into five cases, where each case determines a finite set of congruence classes, whose union equals the set of possible $r_{1}$ values. The problem of bounding the outer integral in (1.8), (1.9) over the set of $x$ such that $r_{1}=$ ord $x$ belongs to exactly one congruence class (for a given $i$ ) then reduces to a routine calculation, given Lemma 2.6 and Remark 2.7, and the bounds from Lemma 2.8 for the corresponding inner integral over the vertical slice at $x$.

To digest the next two definitions, the reader may find it useful to consult Figure 4. It is implicitly assumed throughout (see Lemma 2.3) that $\gamma \mid r_{1}-m_{1}$.

\section{DeFinition 2.4 .}

Case $1\left(i=1, N_{2}>0\right)$. For each $\boldsymbol{\ell}=\left(\ell_{0}, \ell_{1}, \ell_{2}\right) \in\left(\left[0, D^{\prime}\right) \times\left[0, N_{1}^{\prime}\right) \times\left[0, N_{2}^{\prime}\right)\right) \cap \mathbb{Z}_{+}^{3}={ }_{\text {def }} \mathcal{I}_{1}$ set

$$
\mathcal{S}_{1}(\epsilon, \ell)=\left\{r_{1}: r_{1}^{\prime} \eta+m_{2}-\epsilon \equiv \ell_{0}\left(\bmod D^{\prime}\right) ; r_{1}^{\prime} \eta_{1}-f_{1} \equiv \ell_{1}\left(\bmod N_{1}^{\prime}\right) ; e_{1}-r_{1}^{\prime} \eta_{2} \equiv \ell_{2}\left(\bmod N_{2}^{\prime}\right)\right\} .
$$

Case $2\left(i=1, N_{2}=0\right)$. For each $\boldsymbol{\ell}=\left(\ell_{0}, \ell_{1}\right) \in\left(\left[0, D^{\prime}\right) \times\left[0, N_{1}^{\prime}\right)\right) \cap \mathbb{Z}_{+}^{2}={ }_{\text {def }} \mathcal{I}_{2}$ set

$$
\mathcal{S}_{2}(\epsilon, \ell)=\left\{r_{1}: r_{1}^{\prime} \eta+m_{2}-\epsilon \equiv \ell_{0}\left(\bmod D^{\prime}\right) ; r_{1}^{\prime} \eta_{1}-f_{1} \equiv \ell_{1}\left(\bmod N_{1}^{\prime}\right)\right\} .
$$

Case $3\left(i=2, N_{2} \geqslant 0\right)$. For each $\ell=\left(\ell_{0}, \ell_{1}, \ell_{2}\right) \in\left(\left[0, D^{\prime}\right) \times\left[0, N_{1}^{\prime}\right)^{2}\right) \cap \mathbb{Z}_{+}^{3}={ }_{\text {def }} \mathcal{I}_{3}$ set

$$
\mathcal{S}_{3}(\epsilon, \ell)=\left\{r_{1}: r_{1}^{\prime} \eta+m_{2}-\epsilon \equiv \ell_{0}\left(\bmod D^{\prime}\right) ; r_{1}^{\prime} \eta_{1}-f_{2} \equiv \ell_{1}\left(\bmod N_{1}^{\prime}\right) ; r_{1}^{\prime} \eta_{1}-g_{2} \equiv \ell_{2}\left(\bmod N_{1}^{\prime}\right)\right\} .
$$

Case $4\left(i=3, N_{2}>0\right)$. For each $\ell=\left(\ell_{0}, \ell_{1}, \ell_{2}\right) \in\left(\left[0, D^{\prime}\right) \times\left[0, N_{2}^{\prime}\right)^{2}\right) \cap \mathbb{Z}_{+}^{3}={ }_{\text {def }} \mathcal{I}_{4}$ set

$$
\mathcal{S}_{4}(\epsilon, \ell)=\left\{r_{1}: r_{1}^{\prime} \eta+m_{2}-\epsilon \equiv \ell_{0}\left(\bmod D^{\prime}\right) ; e_{3}-r_{1}^{\prime} \eta_{2} \equiv \ell_{1}\left(\bmod N_{2}^{\prime}\right) ; f_{3}-r_{1}^{\prime} \eta_{2} \equiv \ell_{2}\left(\bmod N_{2}^{\prime}\right)\right\} .
$$

Case $5\left(i=3, N_{2}=0\right)$. For each $\ell=\left(\ell_{0}, \ell_{1}\right) \in\left(\left[0, D^{\prime}\right) \times\left[0, N_{1}^{\prime}\right)\right) \cap \mathbb{Z}_{+}^{2}={ }_{\text {def }} \mathcal{I}_{5}$ set

$$
\mathcal{S}_{5}(\epsilon, \ell)=\left\{r_{1}: r_{1}^{\prime} \eta+m_{2}-\epsilon \equiv \ell_{0}\left(\bmod D^{\prime}\right) ; r_{1}^{\prime} \eta_{1}-g_{3} \equiv \ell_{1}\left(\bmod N_{1}^{\prime}\right)\right\} .
$$




\section{On A QUESTION OF Igusa, II}

In addition, for each $j=1, \ldots, 5$, denote the characteristic function of $\mathcal{S}_{j}(\epsilon, \ell)$ by $\Xi_{j}(\epsilon, \ell)$ and set

$$
i_{j}= \begin{cases}1 & \text { if } j=1,2 \\ 2 & \text { if } j=3 \\ 3 & \text { if } j=4,5\end{cases}
$$

Then define

$$
\begin{aligned}
& r_{\ell}^{(j)}\left(r_{1}, \lambda\right)=\min \mathcal{E}^{\left(i_{j}\right)}\left(r_{1}, \lambda\right) \cdot \Xi_{j}(\epsilon, \ell)\left(r_{1}\right) \\
& R_{\ell}^{(j)}\left(r_{1}, \lambda\right)= \begin{cases}\max \mathcal{E}^{\left(i_{j}\right)}\left(r_{1}, \lambda\right) \cdot \Xi_{j}(\epsilon, \ell)\left(r_{1}\right) & \text { if } N_{2}>0 \\
+\infty & \text { if } N_{2}=0\end{cases}
\end{aligned}
$$

It is clear that, for $r_{1} \in \mathcal{S}_{j}(\epsilon, \ell)$,

$$
r_{\ell}^{(j)}\left(r_{1}, \lambda\right)=r_{1}^{\prime} \eta-\left[B_{i_{j}}\right] D^{\prime}+m_{2}, \quad R_{\ell}^{(j)}\left(r_{1}, \lambda\right)=r_{1}^{\prime} \eta-\left\lceil A_{i_{j}}\right\rceil D^{\prime}+m_{2} \quad\left(\text { if } A_{i_{j}}>-\infty\right) .
$$

It is now easy to check that the following holds for each $j$ and $\ell \in \mathcal{I}_{j}$. Verifications are left to the reader. Figure 4 illustrates the conclusions of Lemma 2.5 when $0<\alpha<\beta<\infty$.

LEMma 2.5. We have

(i) For each $\ell \in \mathcal{I}_{1}$, there exist $\kappa_{1}(\ell), \kappa_{1}^{\prime}(\ell) \geqslant 0$ such that

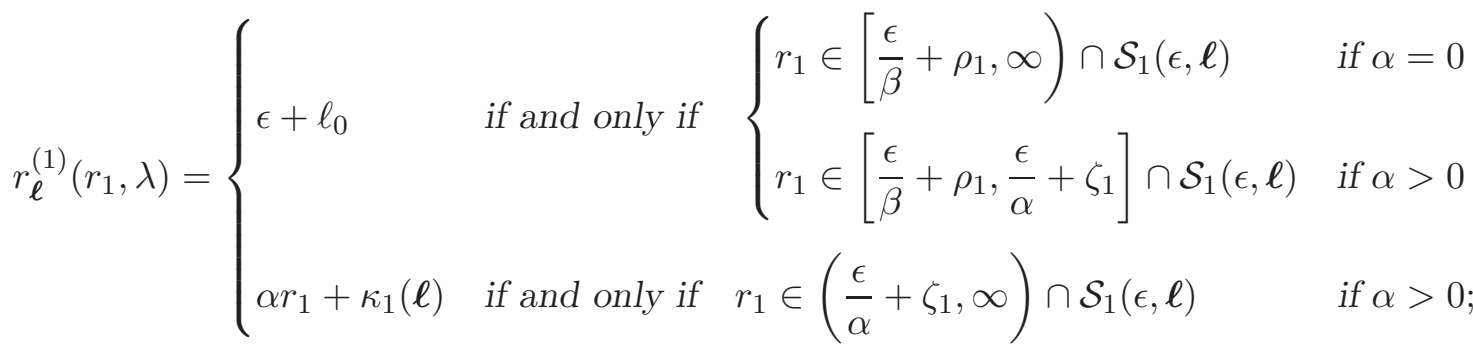

$$
\begin{aligned}
& R_{\ell}^{(1)}\left(r_{1}, \lambda\right)=\beta r_{1}-\kappa_{1}^{\prime}(\ell) \quad \text { if and only if } r_{1} \in\left[\frac{\epsilon}{\beta}+\rho_{1}, \infty\right) \cap \mathcal{S}_{1}(\epsilon, \ell) .
\end{aligned}
$$

(ii) For each $\boldsymbol{\ell} \in \mathcal{I}_{2}$, there exists $\kappa_{2}(\ell) \geqslant 0$ such that

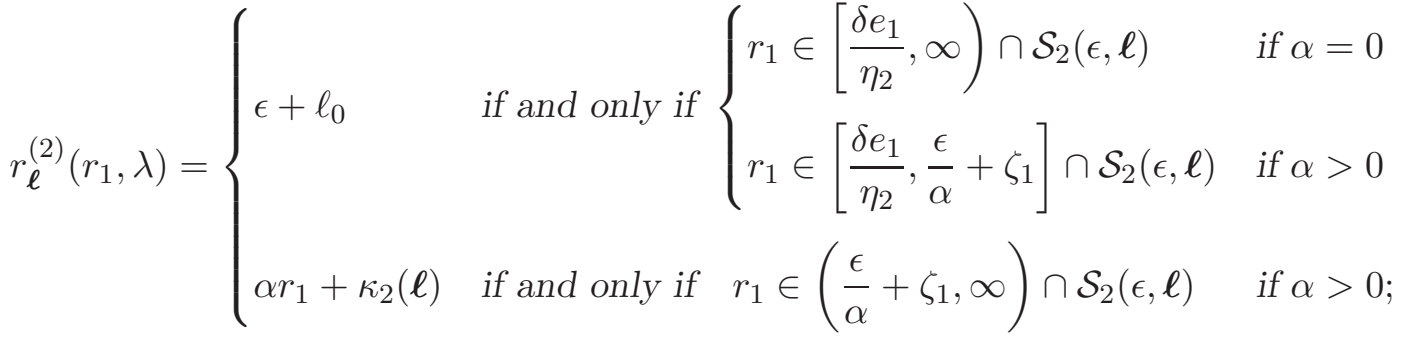

$$
\begin{aligned}
& R_{\ell}^{(2)}\left(r_{1}, \lambda\right)=+\infty \text {. }
\end{aligned}
$$




\section{B. LICHTIN}

(iii) For each $\ell \in \mathcal{I}_{3}$, there exist $\kappa_{3}(\ell), \kappa_{3}^{\prime}(\ell) \geqslant 0$ such that

$$
\begin{aligned}
& r_{\ell}^{(3)}\left(r_{1}, \lambda\right)= \begin{cases}\epsilon+\ell_{0} & \text { if and only if } r_{1} \in\left[\frac{\epsilon}{\alpha}+\rho_{2}, \frac{\epsilon}{\alpha}+\zeta_{2}\right] \cap \mathcal{S}_{3}(\epsilon, \ell) \\
\alpha r_{1}+\kappa_{3}(\ell) & \text { if and only if } r_{1} \in\left(\frac{\epsilon}{\alpha}+\zeta_{2}, \infty\right) \cap \mathcal{S}_{3}(\epsilon, \ell) ;\end{cases} \\
& R_{\ell}^{(3)}\left(r_{1}, \lambda\right)=\alpha r_{1}+\kappa_{3}^{\prime}(\ell) \quad \text { if and only if } r_{1} \in\left[\frac{\epsilon}{\alpha}+\rho_{2}, \infty\right) \cap \mathcal{S}_{3}(\epsilon, \ell) .
\end{aligned}
$$

(iv) For each $\ell \in \mathcal{I}_{4}$, there exist $\kappa_{4}(\ell), \kappa_{4}^{\prime}(\ell) \geqslant 0$ such that

$$
\begin{aligned}
& r_{\ell}^{(4)}\left(r_{1}, \lambda\right)= \begin{cases}\epsilon+\ell_{0} & \text { if and only if } \quad r_{1} \in\left[\frac{\epsilon}{\beta}+\rho_{3}, \frac{\epsilon}{\beta}+\zeta_{3}\right] \cap \mathcal{S}_{4}(\epsilon, \ell) \\
\beta r_{1}-\kappa_{4}(\ell) & \text { if and only if } r_{1} \in\left(\frac{\epsilon}{\beta}+\zeta_{3}, \infty\right) \cap \mathcal{S}_{4}(\epsilon, \ell) ;\end{cases} \\
& R_{\ell}^{(4)}\left(r_{1}, \lambda\right)=\beta r_{1}-\kappa_{4}^{\prime}(\ell) \quad \text { if and only if } r_{1} \in\left[\frac{\epsilon}{\beta}+\rho_{3}, \infty\right) \cap \mathcal{S}_{4}(\epsilon, \ell) .
\end{aligned}
$$

(v) By the definitions given in Definition 2.2, $B_{3}$ can only equal $Q\left(r_{1}, \epsilon\right)$ when $\epsilon \gg 1$. Thus, for any $\ell \in \mathcal{I}_{5}$

$$
\begin{aligned}
& r_{\ell}^{(5)}\left(r_{1}, \lambda\right)=\epsilon+\ell_{0} \quad \text { if and only if } r_{1} \in\left[\frac{e_{3}}{\eta_{2}}+m_{1}, \frac{f_{3}}{\eta_{2}}+m_{1}\right] \cap \mathcal{S}_{5}(\epsilon, \ell) \\
& R_{\ell}^{(5)}\left(r_{1}, \lambda\right)=+\infty
\end{aligned}
$$

Figure 4 illustrates the simple geometry of these assertions when $i=1$. The $r_{2}$ interval depends on the two possible intervals containing the value of $r_{1}$, denoted by $r, r^{\prime}$ (both $r, r^{\prime}$ are understood to belong to $\mathcal{S}_{1}(\epsilon, \ell)$ ). Figure 4 indicates that if $\epsilon / \beta \leqslant r \leqslant \epsilon / \alpha$, then $\alpha r+\kappa_{1}(\ell)<\epsilon$ implies $r_{2} \in \mathcal{E}^{(1)}(r, \lambda)=\left[\epsilon+\ell_{0}, \beta r-\kappa_{1}^{\prime}(\ell)\right] \cap \mathbb{N}$. However, if $r^{\prime}>\epsilon / \alpha$, then $r_{2} \in \mathcal{E}^{(1)}\left(r^{\prime}, \lambda\right)=\left[\alpha r^{\prime}+\right.$ $\left.\kappa_{1}(\ell), \beta r^{\prime}-\kappa_{1}^{\prime}(\ell)\right] \cap \mathbb{N}$. In particular, the endpoints of the $r_{2}$ interval depend explicitly (but in a simple manner) on $\ell$, which serves as an index vector for the arithmetic progression $\mathcal{S}_{1}(\epsilon, \ell)$ containing $r_{1}$.

The final point concerns the structure of the sets $\mathcal{S}_{j}(\epsilon, \ell)$. This, however, is clear by elementary reasoning and is left to the reader.

Lemma 2.6. If $\mathcal{S}_{j}(\epsilon, \ell) \neq \emptyset$, then there exists $L>0$, depending only on $N_{1}^{\prime}, N_{2}^{\prime}, D^{\prime}$, and $c \in[0, L)$, depending on $\epsilon$ and $\boldsymbol{\ell}$, such that $\mathcal{S}_{j}(\epsilon, \boldsymbol{\ell})=\{c+u L: u \geqslant 0\}$.

Remark 2.7. For the applications below, it will not be necessary to be more precise about the modulus $L$ of the arithmetic progression. On the other hand, it will be useful to define the unique integers $u_{j}, U_{j}$ by combining Lemmas 2.5 and 2.6. For each $j \leqslant 4$ and $\boldsymbol{\ell} \in \mathcal{I}_{j}$, there is a partition of $\mathcal{S}_{j}(\epsilon, \ell)$ according to the expression for each $r_{\ell}^{(j)}\left(r_{1}, \lambda\right)$ as follows. Set

$$
\begin{gathered}
\mathcal{S}_{j}^{\prime}(\epsilon, \ell)=\left\{r_{1}: r_{\ell}^{(j)}\left(r_{1}, \lambda\right)=\epsilon+\ell_{0}\right\} \\
\mathcal{S}_{j}^{\prime \prime}(\epsilon, \ell)=\left\{r_{1}: r_{\ell}^{(j)}\left(r_{1}, \lambda\right)=\theta^{\prime} r_{1}+\kappa^{\prime}(\ell)\right\},
\end{gathered}
$$

where, in the notation of Lemma $2.5, j \leqslant 3$ implies $\theta^{\prime}=\alpha$ and $\kappa^{\prime}(\ell)=\kappa_{j}(\ell)$. If $j=4$ then $\theta^{\prime}=\beta$ and $\kappa^{\prime}(\ell)=-\kappa_{4}(\ell)$. In addition, each $\mathcal{S}_{j}^{\prime}$ is finite whenever $\alpha>0$. If $\alpha=0$ (and $j=1,2$ ), or $j=5$, then it is understood that $\mathcal{S}_{j}^{\prime \prime}(\epsilon, \ell)=\emptyset$ for each $\epsilon, \ell$. 
ON A QUESTION OF IGUSA, II

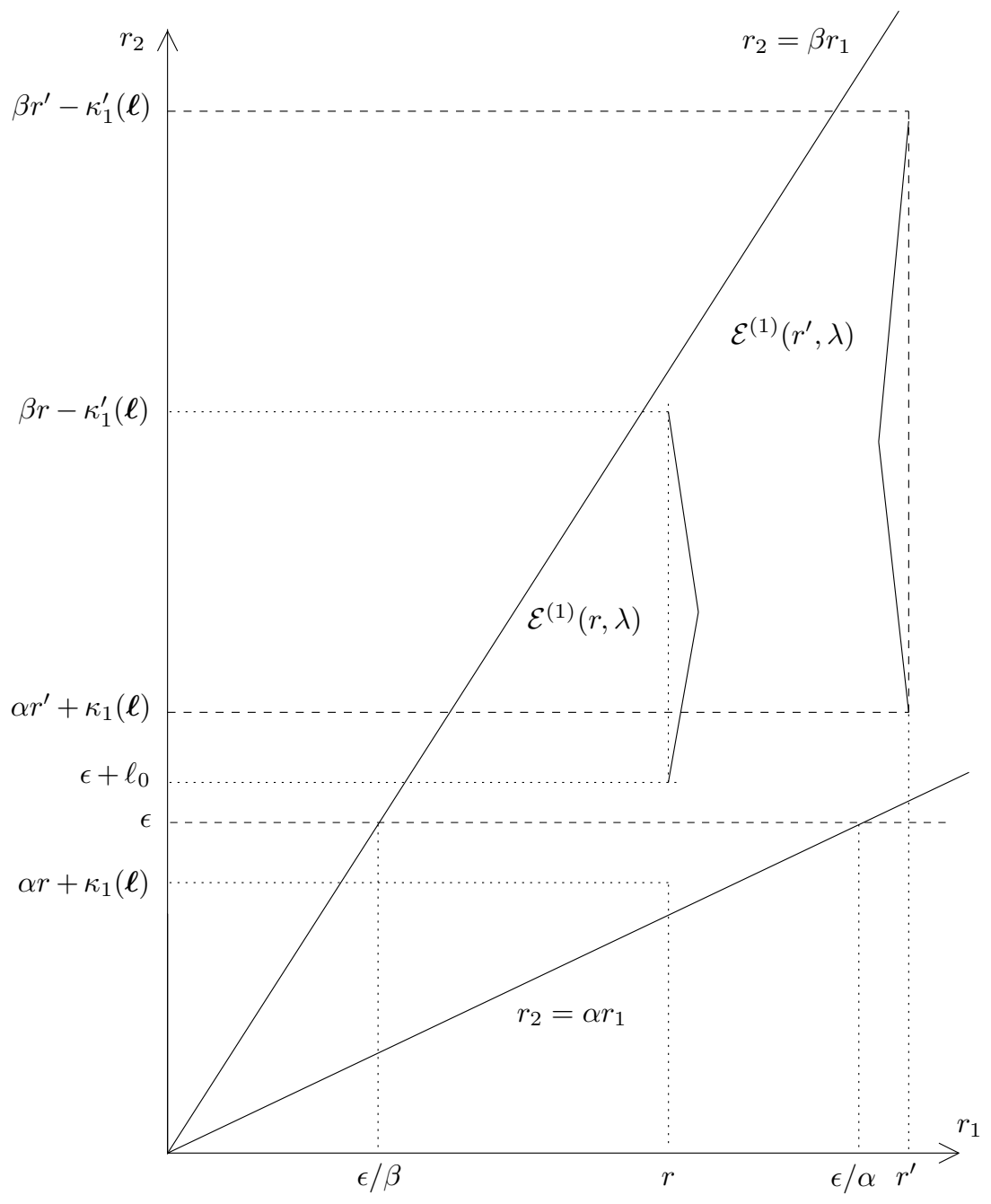

Figure 4.

Using the description of $\mathcal{S}_{j}(\epsilon, \boldsymbol{\ell})$ in Lemma 2.6, define the integers $u_{j}=u_{j}(\epsilon, \boldsymbol{\ell}), U_{j}=U_{j}(\epsilon, \boldsymbol{\ell})$ (when convenient these will be denoted solely by $u_{j}$ and $U_{j}$ ) by setting for any $\epsilon$, each $j$ and $\ell \in \mathcal{I}_{j}$,

$$
\begin{aligned}
& c+u L \in \mathcal{S}_{j}^{\prime}(\epsilon, \ell) \quad \text { if and only if } u_{j} \leqslant u \leqslant U_{j} \\
& c+u L \in \mathcal{S}_{j}^{\prime \prime}(\epsilon, \ell) \quad \text { if and only if } U_{j}+1 \leqslant u .
\end{aligned}
$$

When $\mathcal{S}_{j}^{\prime}$ is unbounded, only $u_{j}$ is defined. In this case, $U_{j}=\infty$. This only occurs if $j=1,2$ and $\alpha=0$, or if $j=5$.

The following is then clear whenever $U_{j}<\infty$.

$(j=1) \quad$ There exist $\Delta_{1}, \Delta_{1}^{\prime} \in[0, L)$ such that

$$
c+u_{1} L=\frac{\epsilon}{\beta}+\rho_{1}+\Delta_{1}, \quad c+U_{1} L=\frac{\epsilon}{\alpha}+\zeta_{1}-\Delta_{1}^{\prime} .
$$

$(j=2)$ There exist $\Delta_{2}, \Delta_{2}^{\prime} \in[0, L)$ such that

$$
c+u_{2} L=\frac{\delta e_{1}}{\eta_{2}}+\Delta_{2}, \quad c+U_{2} L=\frac{\epsilon}{\alpha}+\zeta_{1}-\Delta_{2}^{\prime} .
$$




\section{B. LICHTIN}

$(j=3)$ There exist $\Delta_{3}, \Delta_{3}^{\prime} \in[0, L)$ such that

$$
c+u_{3} L=\frac{\epsilon}{\alpha}+\rho_{2}+\Delta_{3}, \quad c+U_{3} L=\frac{\epsilon}{\alpha}+\zeta_{2}-\Delta_{3}^{\prime} .
$$

$(j=4)$ There exist $\Delta_{4}, \Delta_{4}^{\prime} \in[0, L)$ such that

$$
c+u_{4} L=\frac{\epsilon}{\beta}+\rho_{3}+\Delta_{4}, \quad c+U_{4} L=\frac{\epsilon}{\beta}+\zeta_{3}-\Delta_{4}^{\prime} .
$$

If $U_{j}=\infty$, then the preceding equations hold for each $c+u_{j} L$.

\subsection{Bounds for the inner integral}

In this section $\epsilon=-\operatorname{ord} \lambda-\vartheta_{W}$ is assumed to be unbounded, and the integral over each $E^{(i)}(x, \lambda), i=$ $1,2,3$, is estimated for $\epsilon \gg 1$. To do so, one first expresses the integral as a sum of integrals over the 'walls' of each $E^{(i)}(x, \lambda)$; that is, the union of all cosets $[y]_{\operatorname{ord} y+1}$, where ord $y \in \mathcal{E}^{(i)}(\operatorname{ord} x, \lambda)$. This defines a series, indexed by $\mathcal{E}^{(i)}(\operatorname{ord} x, \lambda)$, whose summands are determined by Claim 2 . Moreover, Lemma 2.3 has shown that the indexing set for the summation is a segment of an arithmetic progression, and Lemma 2.5 has precisely determined the endpoints of each segment, provided ord $x$ is confined to exactly one arithmetic progression $\mathcal{S}_{j}(\epsilon, \ell)$. A standard one variable calculation then shows that the integral over each $E^{(i)}(x, \lambda)$ is bounded by an expression that depends only on the smallest and largest (if finite) elements of this segment. The result is as follows, using Remark 2.7 (also see (3.3)).

LEMma 2.8. For each $j=1, \ldots, 5$, the following hold.

(i) Assume that $r_{1} \in \mathcal{S}_{j}^{\prime}(\epsilon, \ell)$ is such that

$$
r_{\ell}^{(j)}\left(r_{1}, \lambda\right)=\epsilon+\ell_{0} \quad \text { and } \quad R_{\ell}^{(j)}\left(r_{1}, \lambda\right)=\theta r_{1}+\kappa(\ell)
$$

where $\theta \in\{\alpha, \beta\}$ and $\kappa(\ell)$ is some constant (i.e., independent of $\left.r_{1}, \lambda\right)$. Then there exist constants $a_{e}(\ell), d_{e}(\ell), e=0, \ldots, b$, such that for any $\chi_{2}$ and $x$ with ord $x=r_{1}$

$\left.\left.\left|\int_{E^{\left(i_{j}\right)}(x, \lambda)}\right| y\right|^{-v_{2}-1} \log ^{b}|y| \chi_{2}(\operatorname{ac} y) \Psi(\lambda y)|d y||\ll| x\right|^{-\theta v_{2}} \sum_{e} a_{e}(\ell) \log ^{e}|x|+|\lambda|^{v_{2}} \sum_{e} d_{e}(\ell) \log ^{e}|\lambda|$,

where $\ll$ means (here and in (ii)) that the implicit constant is independent of $x, \lambda$. If, however, $R_{\ell}^{(j)}=+\infty$, then the left-most summand equals zero, and the integral is bounded by the right-most summand (depending on $|\lambda|$ ).

(ii) Assume that $r_{1} \in \mathcal{S}_{j}^{\prime \prime}(\epsilon, \ell)$ is such that

$$
r_{\ell}^{(j)}\left(r_{1}, \lambda\right)=\theta^{\prime} r_{1}+\kappa^{\prime}(\ell) \text { and } R_{\ell}^{(j)}\left(r_{1}, \lambda\right)=\theta^{\prime \prime} r_{1}+\kappa^{\prime \prime}(\ell)
$$

where $\theta^{\prime} \leqslant \theta^{\prime \prime}<\infty, \theta^{\prime}, \theta^{\prime \prime} \in\{\alpha, \beta\}$, and $\kappa^{\prime}(\ell), \kappa^{\prime \prime}(\ell)$ are constants. Then there exist constants $a_{e}^{\prime \prime}(\ell), d_{e}^{\prime}(\ell), e=0, \ldots, b$ such that for any $\chi_{2}$ and $x$ with ord $x=r_{1}$,

$$
\begin{aligned}
& \left.\left|\int_{E^{\left(i_{j}\right)}(x, \lambda)}\right| y\right|^{-v_{2}-1} \log ^{b}|y| \chi_{2}(\operatorname{ac} y) \Psi(\lambda y)|d y| \mid \\
& \quad \ll|x|^{-\theta^{\prime \prime} v_{2}} \sum_{e} a_{e}^{\prime \prime}(\ell) \log ^{e}|x|+|x|^{-\theta^{\prime} v_{2}} \sum_{e} d_{e}^{\prime}(\ell) \log ^{e}|x| .
\end{aligned}
$$

If, however, $R_{\ell}^{(j)}=+\infty$, then the left-most summand equals zero, and the integral is bounded by the right-most summand.

(iii) If $\theta=\theta^{\prime \prime}$ and $\kappa(\ell)=\kappa^{\prime \prime}(\ell)$, then $a_{e}(\ell)=a_{e}^{\prime \prime}(\ell)$ for each $e$.

Proof. (i) In the notation of Definition 2.4, the hypothesis means that $B_{i_{j}}=Q\left(r_{1}, \epsilon\right)$ determines the smallest element of $\mathcal{E}^{\left(i_{j}\right)}\left(r_{1}, \lambda\right)$. Assume first that $R_{\ell}^{(j)}\left(r_{1}, \lambda\right)<\infty$ (equivalently, $A_{i_{j}}>-\infty$ ). 


\section{ON A QUESTION OF IGUSA, II}

Thus, the interval for $q$ in Lemma 2.3 equals $\left[\left\lceil A_{i_{j}}\right],\left[B_{i_{j}}\right]\right]$. Since $j$ and $i_{j}$ are fixed below, these indices are dropped from the notation for simplicity.

One now observes that Claims 1 and 2 trivially imply

$$
\left.\left|\int_{\substack{y \in E(x, \lambda) \\ \text { ord } y=r_{2}}}\right| y\right|^{-v_{2}-1} \log ^{b}|y| \chi_{2}(\operatorname{ac} y) \Psi(\lambda y)|d y| \mid \leqslant\left(\hat{T}_{W} \cdot p^{-\vartheta_{W}}\right) \cdot p^{r_{2} v_{2}} r_{2}^{b},
$$

where $\hat{T}_{W}$ is the number of distinct units $\bmod p^{\vartheta_{W}}$ that are congruent $\bmod p^{2 \delta+1}$ to the second component of some $\mathbf{b}_{j}$ that appears in the disjoint union of Claim 1.

Set $C=\hat{T}_{W} \cdot p^{-\vartheta_{W}}$. Since $y \in E(x, \lambda)$ if and only if ord $y \in \mathcal{E}(\operatorname{ord} x, \lambda)$, it then follows that

$$
\left.\left|\int_{E(x, \lambda)}\right| y\right|^{-v_{2}-1} \log ^{b}|y| \chi_{2}(\operatorname{ac} y) \Psi(\lambda y)|d y| \mid \leqslant C \cdot \sum_{\lceil A\rceil \leqslant q \leqslant[B]} p^{\left(r_{1}^{\prime} \eta+m_{2}-q D^{\prime}\right) v_{2}}\left(r_{1}^{\prime} \eta+m_{2}-q D^{\prime}\right)^{b} .
$$

A standard use of partial summation implies that there exist constants $a_{e}, d_{e}$ depending only on $v_{2}, b$, such that for all $\chi_{2}$ and $x$,

$$
\begin{gathered}
\left.\left|\int_{E(x, \lambda)}\right| y\right|^{-v_{2}-1} \log ^{b}|y| \chi_{2}(\operatorname{ac} y) \Psi(\lambda y)|d y| \mid \\
\leqslant C \cdot \sum_{\lceil A\rceil \leqslant q \leqslant[B]} p^{\left(r_{1}^{\prime} \eta+m_{2}-q D^{\prime}\right) v_{2}}\left(r_{1}^{\prime} \eta+m_{2}-q D^{\prime}\right)^{b} \\
=C \cdot\left\{p^{\left(r_{1}^{\prime} \eta+m_{2}-([B]+1) D^{\prime}\right) v_{2}} \cdot \sum_{e=0}^{b} a_{e}\left(r_{1}^{\prime} \eta+m_{2}-([B]+1) D^{\prime}\right)^{e}\right. \\
\left.-p^{\left(r_{1}^{\prime} \eta+m_{2}-\lceil A\rceil D^{\prime}\right) v_{2}} \cdot \sum_{e=0}^{b} d_{e}\left(r_{1}^{\prime} \eta+m_{2}-\lceil A\rceil D^{\prime}\right)^{e}\right\} .
\end{gathered}
$$

By Lemma 2.5, it follows that

$$
r_{1}^{\prime} \eta+m_{2}-([B]+1) D^{\prime}=\left(\epsilon+\ell_{0}\right)-D^{\prime}, \quad r_{1}^{\prime} \eta+m_{2}-\lceil A\rceil D^{\prime}=\theta r_{1}+\kappa(\ell) .
$$

Substituting these expressions into the third and fourth lines in (2.9), one sees that the coefficient of $p^{- \text {ord } \lambda v_{2}}=|\lambda|^{v_{2}}$ (respectively $p^{\theta v_{2} r_{1}}=|x|^{-\theta v_{2}}$ ) equals

$p^{\left(\ell_{0}-D^{\prime}-\vartheta_{W}\right) v_{2}} \cdot \sum_{e=0}^{b} a_{e}\left(-D^{\prime}-\vartheta_{W}+\ell_{0}+\log |\lambda|\right)^{e} \quad\left(\right.$ respectively $\left.-p^{\kappa(\ell) v_{2}} \cdot \sum_{e=0}^{b} d_{e}(-\theta \log |x|+\kappa(\boldsymbol{\ell}))^{e}\right)$.

A straightforward simplification then yields the expression asserted by (i). Here, the coefficients are written $a_{e}(\boldsymbol{\ell}), d_{e}(\boldsymbol{\ell})$ so as to emphasize dependence on $\boldsymbol{\ell}$. (In fact, $a_{e}(\boldsymbol{\ell})$ depends on $v_{2}, b, \ell_{0}$, and $d_{e}(\boldsymbol{\ell})$ depends on $v_{2}, b, \boldsymbol{\ell}$. Since the dependence on $\boldsymbol{\ell}$ is the most significant, this is emphasized in the notation.)

An analogous argument applies if $R_{\ell}\left(r_{1}, \lambda\right)=+\infty$. The letter $a$ will continue to be used to denote the coefficients that depend on $\boldsymbol{\ell}$. (The actual values will differ of course from the preceding $a_{e}(\ell)$.) One first observes that $R_{\ell}\left(r_{1}, \lambda\right)=+\infty$ implies $N_{2}=0$. Thus, the value of $v_{2}$ must equal the $s_{2}$ axis intercept of the line $\mathcal{L}_{2}={ }_{\text {def }}\left\{M_{2} s_{2}+\mu_{2}=0\right\}$ (see (1.6)). As a result, $v_{2}<0$, which implies the exponent of $|y|$ in (2.9) is larger than -1 . So, the integral converges absolutely 


\section{B. LICHTIN}

since $\lim _{q \rightarrow-\infty} v_{2}\left(r_{1}^{\prime} \eta+m_{2}-q D^{\prime}\right)=-\infty$. One next concludes by Lemma A.3, that

$$
\begin{aligned}
& \left.\left|\int_{E(x, \lambda)}\right| y\right|^{-v_{2}-1} \log ^{b}|y| \chi_{2}(\operatorname{ac} y) \Psi(\lambda y)|d y| \mid \\
& \leqslant C \cdot \sum_{-\infty<q \leqslant[B]} p^{\left(r_{1}^{\prime} \eta+m_{2}-q D^{\prime}\right) v_{2}}\left(r_{1}^{\prime} \eta+m_{2}-q D^{\prime}\right)^{b} \\
& \quad=C \cdot p^{\left(r_{1}^{\prime} \eta+m_{2}-([B]+1) D^{\prime}\right) v_{2}} \cdot \sum_{e=0}^{b} a_{e}\left(r_{1}^{\prime} \eta+m_{2}-([B]+1) D^{\prime}\right)^{e} .
\end{aligned}
$$

Since $r_{1}^{\prime} \eta+m_{2}-([B]+1) D^{\prime}=\left(\epsilon+\ell_{0}\right)-D^{\prime}$ remains as above, the preceding argument applies to complete the proof.

The proof of (ii) is similar, using (2.9), the fact that

$$
r_{1}^{\prime} \eta+m_{2}-([B]+1) D^{\prime}=\theta^{\prime} r_{1}+\kappa^{\prime}(\ell), \quad r_{1}^{\prime} \eta+m_{2}-\lceil A\rceil D^{\prime}=\theta^{\prime \prime} r_{1}+\kappa^{\prime \prime}(\ell),
$$

and

$$
p^{\left(r_{1}^{\prime} \eta+m_{2}-\lceil A\rceil D^{\prime}\right) v_{2}}=|x|^{-\theta^{\prime \prime} v_{2}} \cdot p^{\kappa^{\prime \prime}(\ell) v_{2}} .
$$

Using (2.11) in place of (2.10), the proof of (ii) when $R_{\ell}\left(r_{1}, \lambda\right)=+\infty$ is the same as that in (i).

The proof of (iii) follows immediately from the fact that the $a_{e}$ only depend on $v_{2}, b$, and the assumption that $\theta^{\prime \prime} r_{1}+\kappa^{\prime \prime}(\ell)=\theta r_{1}+\kappa(\ell)$.

Remark 2.9. Since one does not a priori know whether, in Claim $1, \bigsqcup_{j}\left[\mathbf{b}_{j}\right]_{2 \delta+1}=\mathcal{U}_{p}^{2}$, it appears to be difficult to precisely evaluate the inner integral. To do so would require a better understanding of

the exponential sum $\sum_{j=1}^{T} \chi_{2}\left(b_{j, 2}\right) \Psi\left(p^{e} \lambda b_{j, 2}\right)$ for each $e$ such that $e+\operatorname{ord} \lambda \in\left[-\vartheta_{W},-1\right]$. One case in which this is (fortunately) possible is discussed in $\S 4.2$.

\section{Decay rates for the integrals (1.8) and (1.9)}

This section completes the proof of Theorem A by establishing the purely local bounds (1.8), (1.9) for a given $\boldsymbol{\omega}=(\iota, \mathbf{M}, \mathbf{b}, \boldsymbol{\chi}) \in \mathcal{Y}_{W}$. Recall that $(f, g)=\mathbf{P} \circ \theta-\mathbf{P} \circ \theta(\boldsymbol{x})$ when $W=\theta U(\boldsymbol{x})$, and $\Omega_{k}=\left\{\boldsymbol{\lambda}:|\boldsymbol{\lambda}|=\left|\lambda_{k}\right|\right\}$.

THEOREM 1. We have the following.

(i) If $\boldsymbol{x}$ is a good point for $(f, g)$, then $\mathcal{M}_{\boldsymbol{\omega}}^{*}(\boldsymbol{\lambda})=O_{\varepsilon}\left(|\boldsymbol{\lambda}|^{\sigma_{\iota}+\varepsilon}\right)$, where $\sigma_{\iota}=\max _{j}\left\{\sigma_{j}\right.$-axis intercept of $\left.\Gamma_{\iota}\right\}$.

(ii) If $\boldsymbol{x}$ is bad for $(f, g)$, then for each $k,\left.\int \mathcal{M}_{\boldsymbol{\omega}}(\boldsymbol{\tau}) \Psi\left(\boldsymbol{\lambda} \cdot \zeta_{k}(\boldsymbol{\tau})\right)|d \boldsymbol{\tau}|\right|_{\Omega_{k}}=O_{\varepsilon}\left(\left|\lambda_{k}\right|^{\sigma_{2}\left(\Gamma_{\iota, k}\right)+\varepsilon}\right)$, where $\sigma_{2}\left(\Gamma_{\iota, k}\right)$ is the $\sigma_{2}$-axis intercept of $\Gamma_{\iota, k}$.

An immediate consequence of Theorem 1 is the following characterization of the local decay rate $\alpha(W)$ for $G_{W}(\boldsymbol{\lambda})$ in terms of the geometry of the polygons $\Gamma(W), \Gamma_{k}(W)$ (see Definition 1.7).

Theorem 2. We have the following.

(i) If $\boldsymbol{x}$ is a good point for $(f, g)$, then $G_{W}(\boldsymbol{\lambda})=O_{\varepsilon}\left(|\boldsymbol{\lambda}|^{\sigma(\Gamma)+\varepsilon}\right)$.

(ii) If $\boldsymbol{x}$ is a bad point for $(f, g)$, then for each $k,\left.G_{W}(\boldsymbol{\lambda})\right|_{\Omega_{k}}=O_{\varepsilon}\left(\left|\lambda_{k}\right|^{\sigma_{2}\left(\Gamma_{k}\right)+\varepsilon}\right)$, where $\sigma_{2}\left(\Gamma_{k}\right)$ is the $\sigma_{2}$-axis intercept of $\Gamma_{k}(W)$.

Before proceeding to the proof, some remarks will be useful.

Remark 3.1. The proof of Theorem 1 is the same for parts (i) and (ii). It is based on the discussion in $\S 2$, which applied uniformly to the pair $(f, g)$ in $\Omega_{2},(g, f)$ in $\Omega_{1}$, or each $\left(F_{k}, G_{k}\right)$ in $\Omega_{k}$. 


\section{On A QUESTION OF Igusa, II}

By Definition 2.2, it therefore suffices to prove part (i) in $\Omega_{2}$ with the exponent given by $\sigma_{2}\left(\Gamma_{\iota}\right)$. The extension to $\Omega_{1}$ is done by interchanging $f$ with $g$, and using the fact that the $\sigma_{2}$-axis intercept of the polygon

$$
\hat{\Gamma}_{\iota}=\partial\left(\bigcap_{i=1}^{2}\left\{\left(\sigma_{1}, \sigma_{2}\right) \in(-\infty, 0]^{2}: M_{i} \sigma_{1}+N_{i} \sigma_{2} \geqslant-\mu_{i}\right\}\right)
$$

equals the $\sigma_{1}$-axis intercept of $\Gamma_{\iota}$. The proof of (i) will therefore use the discussion of $\S 2$ by setting $\lambda=\lambda_{2}$, and $\boldsymbol{t}=\mathbf{t}=(f, g)$ (i.e. $\left.(x, y)=\left(t_{1}, t_{2}\right)\right)$. The extension to $\Omega_{1}$ then follows by setting $\lambda=\lambda_{1}, \boldsymbol{t}=\mathbf{t}=(g, f)$. To prove part (ii) in $\Omega_{k}$ one chooses $\lambda=\lambda_{k}$ and $\boldsymbol{t}=\boldsymbol{\tau}=\left(F_{k}, G_{k}\right)$ (i.e. $\left.(x, y)=\left(\tau_{1}, \tau_{2}\right)\right)$. In either case, $\epsilon=-\operatorname{ord} \lambda-\vartheta_{W}$ is unbounded in the appropriate cone. Set $k^{\prime}$ to denote the complementary index to $k$ in the sense that $\left\{k, k^{\prime}\right\}=\{1,2\}$.

Remark 3.2. For the reader's convenience, It is useful to record here the expressions for the axis intercepts of $\Gamma_{\iota}$. This depends on the quadrant containing the intersection point $v\left(=\mathcal{L}_{1} \cap \mathcal{L}_{2}\right)$ (see (1.6) and Figure 1) as follows:

$$
\begin{gathered}
v_{1}<0, v_{2} \geqslant 0 \quad \text { implies } \beta<\infty, \quad \sigma_{j}\left(\Gamma_{\iota}\right)=\sigma_{j}\left(\mathcal{L}_{2}\right), \quad j=1,2, \\
\text { and } \sigma_{\iota}=\max \left\{v_{1}+\beta v_{2}, \frac{v_{1}}{\beta}+v_{2}\right\} ; \\
v_{1}, v_{2}<0 \quad \text { implies } \sigma_{1}\left(\Gamma_{\iota}\right)=\sigma_{1}\left(\mathcal{L}_{1}\right), \quad \sigma_{2}\left(\Gamma_{\iota}\right)=\sigma_{2}\left(\mathcal{L}_{2}\right), \\
\text { and } \sigma_{\iota}=\left\{\begin{array}{l}
\max \left\{v_{1}+\alpha v_{2}, \frac{v_{1}}{\beta}+v_{2}\right\} \quad \text { if } \beta<\infty \\
\max \left\{v_{1}+\alpha v_{2}, v_{2}\right\} \quad \text { if } \beta=+\infty ;
\end{array}\right. \\
v_{1} \geqslant 0, v_{2}<0 \quad \operatorname{implies} \alpha>0, \quad \sigma_{j}\left(\Gamma_{\iota}\right)=\sigma_{j}\left(\mathcal{L}_{1}\right), \quad j=1,2, \\
\text { and } \sigma_{\iota}=\max \left\{v_{1}+\alpha v_{2}, \frac{v_{1}}{\alpha}+v_{2}\right\} .
\end{gathered}
$$

By Remark 2.7, for fixed $j=1, \ldots, 5$, the set of possible $r_{1}=$ ord $x$ values is partitioned into the sets $\mathcal{S}_{j}^{\prime}(\epsilon, \ell), \mathcal{S}_{j}^{\prime \prime}(\epsilon, \ell)$ for $\ell \in \mathcal{I}_{j}$. For $* \in\left\{^{\prime}{ }^{\prime \prime},\right\}$, define the following integrals (for simplicity, the domain of integration in $x$ is defined in terms of ord $x$ ) for part (i):

$$
\begin{aligned}
I_{j}^{*}(\boldsymbol{\lambda}, \ell)= & \int_{\operatorname{ord} x \in \mathcal{S}_{j}^{*}(\epsilon, \ell)}|x|^{-v_{1}-1} \log ^{b_{1}}|x| \chi_{1}(\operatorname{ac} x) \Psi\left(\lambda_{1} x\right) \\
& \times\left(\int_{E^{\left(i_{j}\right)}(x, \epsilon)}|y|^{-v_{2}-1} \log ^{b_{2}}|y| \chi_{2}(\operatorname{ac} y) \Psi\left(\lambda_{2} y\right)|d y|\right)|d x| .
\end{aligned}
$$

It is clear that

$$
\mathcal{M}_{\boldsymbol{\omega}}^{*}(\boldsymbol{\lambda})=\sum_{j=1}^{5} \sum_{\ell \in \mathcal{I}_{j}}\left(I_{j}^{\prime}(\boldsymbol{\lambda}, \boldsymbol{\ell})+I_{j}^{\prime \prime}(\boldsymbol{\lambda}, \boldsymbol{\ell})\right)
$$

where the sum is for $j \in\{1,3,4\}$ if $N_{2}>0$ and $j \in\{2,3,5\}$ if $N_{2}=0$.

For given $k$ define for part (ii) (see (1.2c)):

$$
\begin{aligned}
I_{j}^{*}(\boldsymbol{\lambda}, \ell)= & \int_{\operatorname{ord} x \in \mathcal{S}_{j}^{*}\left(\epsilon_{k}, \ell\right)}|x|^{-v_{1}-1} \log ^{b_{1}}|x| \chi_{1}(\operatorname{ac} x) \Psi\left(c_{0} \lambda_{k^{\prime}} x^{\gamma}+\lambda_{k} \psi(x)\right) \\
& \times\left(\int_{E^{\left(i_{j}\right)}\left(x, \lambda_{k}\right)}|y|^{v_{2}-1} \log ^{b_{2}}|y| \chi_{2}(\operatorname{ac} y) \Psi\left(\lambda_{k} y\right)|d y|\right)|d x| .
\end{aligned}
$$




\section{B. LICHTIN}

It is clear that

$$
\int \mathcal{M}_{\boldsymbol{\omega}}(\boldsymbol{\tau}) \Psi\left(\zeta_{k}(\boldsymbol{\tau}) \cdot \boldsymbol{\lambda}\right)|d \boldsymbol{\tau}|=\sum_{j=1}^{5} \sum_{\boldsymbol{\ell} \in \mathcal{I}_{j}}\left(I_{j}^{\prime}(\boldsymbol{\lambda}, \boldsymbol{\ell})+I_{j}^{\prime \prime}(\boldsymbol{\lambda}, \boldsymbol{\ell})\right) .
$$

Thus, it suffices to show the bound in part (i) restricted to $\Omega_{2}$, (respectively part (ii) in $\Omega_{k}$ ) for a single term $I_{j}^{\prime}(\boldsymbol{\lambda}, \boldsymbol{\ell})+I_{j}^{\prime \prime}(\boldsymbol{\lambda}, \boldsymbol{\ell})$, whose summands are defined by (3.1) (respectively (3.2)).

Notation. If $\mathcal{P}$ is any predicate (an expression having a true or false value depending on the value of a certain variable), then denote by $\delta_{\mathcal{P}}$ the function that equals one if the expression is true and equals zero if the expression is false.

Proof of Theorem 1. Throughout the discussion one works with any nonempty and fixed $\mathcal{S}_{j}(\epsilon, \ell)=$ $\{c+u L: u \geqslant 0\}$ and uses the objects (and notations) introduced in Remark 2.7. To estimate $I_{j}^{\prime}+I_{j}^{\prime \prime}$ from above, one uses Lemma 2.8(i) (respectively (ii)) for the inner integral of $I_{j}^{\prime}$ (respectively $I_{j}^{\prime \prime}$ ). As a result, the values of the quantities, denoted by $\theta, \theta^{\prime}, \theta^{\prime \prime}$ in the statement of Lemma 2.8 , need to be specified first, given that $R_{\ell}^{(j)} \neq+\infty$ and $\mathcal{S}_{j}^{\prime \prime}(\epsilon, \ell) \neq \emptyset$. These are as follows:

$$
\begin{array}{ll}
j=1 & \text { implies } \theta=\theta^{\prime \prime}=\beta, \quad \theta^{\prime}=\alpha, \quad \kappa(\ell)=\kappa^{\prime \prime}(\ell)=-\kappa_{1}^{\prime}(\ell), \\
j=3 & \text { implies } \theta=\theta^{\prime}=\theta^{\prime \prime}=\alpha, \quad \kappa(\ell)=\kappa^{\prime \prime}(\ell)=\kappa_{3}^{\prime}(\ell), \\
j=4 & \text { implies } \theta=\theta^{\prime}=\theta^{\prime \prime}=\beta, \quad \kappa(\ell)=\kappa^{\prime \prime}(\ell)=-\kappa_{4}^{\prime}(\ell),
\end{array}
$$

where the indicated expressions for $\kappa(\ell)$ (in terms of $\kappa_{j}^{\prime}(\ell)$ ) are determined by Lemma 2.5. If $R_{\ell}^{(j)}=$ $+\infty$, then $j \in\{2,5\}$. If $j=2$, then Lemmas 2.5 and 2.8(ii) imply $\theta^{\prime}=\alpha$. Recall that $\mathcal{S}_{j}^{\prime \prime}(\epsilon, \ell)=\emptyset$ (i.e. $U_{j}=\infty$ ) can only occur if $\alpha=0$ and $j \in\{1,2\}$, or if $j=5$. Moreover, Lemma 2.5 implies that $j=3$ (respectively $j=4$ ) implies $\alpha>0$ (respectively $\beta<\infty$ ).

It now follows that

$$
\begin{aligned}
&\left|I_{j}^{\prime}(\boldsymbol{\lambda}, \boldsymbol{\ell})+I_{j}^{\prime \prime}(\boldsymbol{\lambda}, \boldsymbol{\ell})\right| \ll \delta_{U_{j}=\infty} \cdot\{ \delta_{j=1} \cdot \sum_{e=0}^{b_{2}} a_{e}(\boldsymbol{\ell}) \cdot \int_{\substack{\operatorname{ord} x=c+u L \\
u \in\left[u_{j}, \infty\right)}}|x|^{-v_{1}-\theta v_{2}-1} \log ^{e+b_{1}}|x||d x| \\
&\left.+|\lambda|^{v_{2}} \sum_{e=0}^{b_{2}} d_{e}(\ell) \log ^{e}|\lambda| \cdot \int_{\substack{\operatorname{ord} x \in \mathcal{S}_{j}^{\prime}(\epsilon, \ell) \\
\text { ord } x=c+u L}}|x|^{-v_{1}-1} \log ^{b_{1}}|x||d x|\right\} \\
&+\delta_{U_{j}<\infty} \cdot\left\{\delta_{j \in\{1,3,4\}} \cdot \sum_{e=0}^{b_{2}} a_{e}(\ell) \cdot \int_{\substack{\operatorname{ord} x=c+u L \\
u \in\left[u_{j}, \infty\right)}}|x|^{-v_{1}-\theta v_{2}-1} \log ^{e+b_{1}}|x||d x|\right. \\
&+|\lambda|^{v_{2}} \sum_{e=0}^{b_{2}} d_{e}(\ell) \log ^{e}|\lambda| \cdot \int_{\substack{\operatorname{ord} x=c+u L \\
u \in\left[u_{j}, U_{j}\right]}}|x|^{-v_{1}-1} \log ^{b_{1}}|x||d x| \\
&\left.+\sum_{e=0}^{b_{2}} d_{e}^{\prime}(\ell) \int_{\substack{\operatorname{ord} x=c+u L \\
u \in\left[U_{j}+1, \infty\right)}}|x|^{-v_{1}-\theta^{\prime} v_{2}-1} \log ^{e+b_{1}}|x||d x|\right\} .
\end{aligned}
$$

Remark. The improper integrals, whose integrands contain a factor $|x|^{-v_{1}-\vartheta v_{2}-1}, \vartheta \in\left\{\theta, \theta^{\prime}\right\}$, and are determined by the conditions $u \in\left[u_{j}, \infty\right)$ and $u \in\left[U_{j}+1, \infty\right)$, converge absolutely. This follows from the fact that for any value of $\theta, \theta^{\prime}, v_{1}+\theta v_{2}$ and $v_{1}+\theta^{\prime} v_{2}$ are axis intercepts of the lines $\mathcal{L}_{1}, \mathcal{L}_{2}$ and, thus, must be strictly negative. As a result, if $U_{j}<\infty$, then the two contributions over $u \in\left[u_{j}, U_{j}\right]$ and $u \in\left[U_{j}+1, \infty\right)$ with integrand $|x|^{-v_{1}-\theta v_{2}-1} \log ^{e+b_{1}}|x|$ can be added together by 


\section{ON A QUESTION OF IGUSA, II}

Lemma 2.8(iii) and (3.3) to equal one integral over $\left\{x:\right.$ ord $\left.x=c+u L, u \in\left[u_{j}, \infty\right)\right\}$. The only other type of improper integral occurs when $j=2, U_{2}=\infty$, and $\mathcal{S}_{2}^{\prime}(\epsilon, \boldsymbol{\ell})$ is unbounded. Since these conditions occur exactly when $\beta=+\infty$ and $\alpha=0$, it must be the case that $v_{1}<0$, so that the integral does converge absolutely. Note that Lemma 2.5 ensures that if $j=5$ then $\mathcal{S}_{5}^{\prime}(\epsilon, \ell)$ is bounded, so the integral is not improper.

One next estimates each integral in (3.4). To do so, one now uses Remark 2.7 to express $c+u L$ in terms of $\epsilon, \alpha, \beta$ when $u=u_{j}$ or $u=U_{j}+1$. This earlier discussion implies the following:

$$
\begin{gathered}
c+u_{j} L= \begin{cases}\frac{\epsilon}{\beta}+O(1) & \text { if } j=1,4 \\
\frac{\epsilon}{\alpha}+O(1) & \text { if } j=3 \\
O(1) & \text { if } j=2,5 ;\end{cases} \\
c+\left(U_{j}+1\right) L= \begin{cases}\frac{\epsilon}{\alpha}+O(1) & \text { if } j=1,2,3 \text { and } \mathcal{S}_{j}^{\prime \prime} \neq \emptyset \\
\frac{\epsilon}{\beta}+O(1) & \text { if } j=4 \text { and } \mathcal{S}_{j}^{\prime \prime} \neq \emptyset \\
\infty & \text { if } \left.j=5 \text { (since } \mathcal{S}_{j}^{\prime \prime}=\emptyset\right),\end{cases}
\end{gathered}
$$

where $O(1)$ denotes a function (in particular, of $\epsilon$ though there is also dependence on $j, \boldsymbol{\ell}$, of course) that is bounded uniformly in $\epsilon$ as $\epsilon \rightarrow \infty$. Using Lemma 2.5, Remark 2.7, and (3.5), the estimates for (3.4) are straightforward and are left to the reader to verify as a useful exercise. For convenience they are organized into the following four claims.

Claim 3. For each $j \in\{1,3,4\}$ and $i=0,1, \ldots, b_{1}+b_{2}$,

$$
\delta_{U_{j}<\infty} \cdot \delta_{j \in\{1,3,4\}} \int_{\substack{\operatorname{ord} x=c+u L \\ u \in\left[u_{j}, \infty\right)}}|x|^{-v_{1}-\theta v_{2}-1} \log ^{i}|x||d x|= \begin{cases}O_{\varepsilon}\left(|\lambda|^{v_{1} / \beta+v_{2}+\varepsilon}\right) & \text { if } j=1,4 \\ O_{\varepsilon}\left(|\lambda|^{v_{1} / \alpha+v_{2}+\varepsilon}\right) & \text { if } j=3 .\end{cases}
$$

Similarly,

$$
\delta_{U_{1}=\infty} \cdot \int_{\substack{u \in\left[u_{1}, \infty\right) \\ \operatorname{ord} x=c+u L}}|x|^{-v_{1}-\theta v_{2}-1} \log ^{i}|x||d x|=O_{\varepsilon}\left(|\lambda|^{v_{1} / \beta+v_{2}+\varepsilon}\right) .
$$

Claim 4. For each $j \leqslant 4$ and $i=0,1, \ldots, b_{1}$,

$$
\begin{gathered}
\delta_{U_{j}<\infty} \cdot|\lambda|^{v_{2}} \sum_{e=0}^{b_{2}} d_{e}(\ell) \log ^{e}|\lambda| \cdot \int_{\substack{\operatorname{ord} x=c+u L \\
u \in\left[u_{j}, U_{j}\right]}}|x|^{-v_{1}-1} \log ^{i}|x||d x| \\
= \begin{cases}O_{\varepsilon}\left(\max \left\{|\lambda|^{v_{1} / \alpha+v_{2}+\varepsilon},|\lambda|^{v_{1} / \beta+v_{2}+\varepsilon}\right\}\right) & \text { if } j=1 \\
O_{\varepsilon}\left(\max \left\{|\lambda|^{v_{2}+\varepsilon},|\lambda|^{v_{1} / \alpha+v_{2}+\varepsilon}\right\}\right) & \text { if } j=2 \\
O_{\varepsilon}\left(|\lambda|^{v_{1} / \alpha+v_{2}+\varepsilon}\right) & \text { if } j=3 \\
O_{\varepsilon}\left(|\lambda|^{v_{1} / \beta+v_{2}+\varepsilon}\right) & \text { if } j=4 .\end{cases}
\end{gathered}
$$

Claim 5. For each $j \leqslant 4$ and $i=0,1, \ldots, b_{1}+b_{2}$,

$$
\delta_{U_{j}<\infty} \cdot \int_{\substack{\operatorname{ord} x=c+u L \\ u \in\left[U_{j}+1, \infty\right)}}|x|^{-v_{1}-\theta^{\prime} v_{2}-1} \log ^{i}|x||d x|= \begin{cases}O_{\varepsilon}\left(|\lambda|^{v_{1} / \alpha+v_{2}+\varepsilon}\right) & \text { if } j=1,2,3 \\ O_{\varepsilon}\left(|\lambda|^{v_{1} / \beta+v_{2}+\varepsilon}\right) & \text { if } j=4 .\end{cases}
$$




\section{B. LICHTIN}

Claim 6. For each $j \in\{1,2,5\}$,

$$
\begin{aligned}
\delta_{U_{j}=\infty} & \cdot|\lambda|^{v_{2}} \sum_{e=0}^{b_{2}} d_{e}(\ell) \log ^{e}|\lambda| \cdot \int_{\begin{array}{c}
\operatorname{ord} x \in \mathcal{S}_{j}^{\prime}(\epsilon, \ell) \\
\text { ord } x=c+u L \\
u \in\left[u_{j}, \infty\right)
\end{array}}^{\operatorname{or}}|x|^{-v_{1}-1} \log ^{b_{1}}|x||d x| \\
& = \begin{cases}O_{\varepsilon}\left(|\lambda|^{v_{1} / \beta+v_{2}+\varepsilon}\right) & \text { if } j=1 \\
O_{\varepsilon}\left(|\lambda|^{v_{2}+\varepsilon}\right) & \text { if } j=2,5 .\end{cases}
\end{aligned}
$$

Checking the expressions in Remark 3.2, one verifies that the exponent of $|\lambda|$ in the bounds of each of these claims is always at most the $\sigma_{2}$-axis intercept of the appropriate polygon, $\Gamma_{\iota}$ or $\Gamma_{\iota, k}$, plus an arbitrary $\varepsilon$. This completes the proof of Theorem 1 .

\section{Condition $P^{(2)}$ and an improvement of Theorem 2}

The estimate in Theorem 2(i) will be $O_{\varepsilon}\left(|\boldsymbol{\lambda}|^{-1+\varepsilon}\right)$ if at least one side of $\Gamma(W)$ lies on the line $\sigma_{1}=-1$ or $\sigma_{2}=-1$. The same occurs for the estimates in (ii) if the $\sigma_{2}$-axis intercept of either polygon $\Gamma_{k}(W)$ equals -1 . For arithmetic purposes, this estimate is too weak to be used for any global purpose. This section introduces a geometric property, which, if satisfied, can often help lead to a significantly better decay rate in $\max \left\{\left|\lambda_{1}\right|,\left|\lambda_{2}\right|\right\}$. Igusa identified a (local) property in the case of one polynomial and called it 'Condition P' [Igu78, p. 154]. There is a natural analog of this condition for two polynomials.

Definition of Condition $P^{(2)}$. Let $W=\theta U(\boldsymbol{x})$ be a good $\mathbf{P}$ wedge. Assume that $\boldsymbol{x}$ is a good point for $(f, g)(=\mathbf{P} \circ \theta-\mathbf{P} \circ \theta(\boldsymbol{x}))$. Then Condition $P^{(2)}$ is satisfied at $\boldsymbol{x}$ if and only if the matrix $\mathbf{A}(\boldsymbol{x})$ (see Definition 1.7) contains at most one column vector proportional to

$$
\hat{\mathbf{e}}_{1}=\left(\begin{array}{l}
1 \\
0 \\
1
\end{array}\right) \quad \text { or } \quad \hat{\mathbf{e}}_{2}=\left(\begin{array}{l}
0 \\
1 \\
1
\end{array}\right) .
$$

If a column vector $\mathbf{c}$ of $\mathbf{A}(\boldsymbol{x})$ is proportional to $\hat{\mathbf{e}}_{i}$, then, in fact, $\mathbf{c}=\hat{\mathbf{e}}_{i}$.

If $\boldsymbol{x}$ is bad for $(f, g)$ in the sense of Remarks $1.2(\mathrm{iii})$, and $\left(F_{k}, G_{k}\right) \rightarrow(f, g)$ is an amelioration, then Condition $P^{(2)}$ is satisfied at $\boldsymbol{x}$ if the preceding property holds for the matrix $\mathbf{A}_{k}(\boldsymbol{x})$.

Remark. The argument in Appendix B shows that if Condition $P^{(2)}$ holds for one value of $k$, then it also holds for the other possible value of $k$.

Throughout this section $W$ will be a good $\mathbf{P}$ wedge with presentation $W=\theta U(\boldsymbol{x})$, Condition $P^{(2)}$ will be assumed to hold at $\boldsymbol{x}$, and $(f, g)=\mathbf{P} \circ \theta-\mathbf{P} \circ \theta(\boldsymbol{x})$.

\subsection{Statement of the main result}

Let $\mathbf{A}$ denote the matrix $\mathbf{A}(\boldsymbol{x})$ if $\boldsymbol{x}$ is good for $(f, g)$ (respectively $\mathbf{A}_{k}(\boldsymbol{x})$ if $\boldsymbol{x}$ is good for $\left(F_{k}, G_{k}\right)$ ). By a permutation of local coordinates at $\boldsymbol{x}$, one can always arrange the nonzero columns of the matrix $\mathbf{A}$ so that if $\hat{\mathbf{e}}_{1}$ (respectively $\hat{\mathbf{e}}_{2}$ ) is a column vector of $\mathbf{A}$ then it is the left-most (respectively right-most) column. Let $R$ be the number of nonzero columns of $\mathbf{A}, \iota=\{i, R\} \in \mathcal{I}_{0}$ (see (1.6)), and $\boldsymbol{\omega}=(\iota, \mathbf{M}, \mathbf{b}, \boldsymbol{\chi}) \in \mathcal{Y}_{W}$. Set $\sigma_{j}\left(\mathcal{L}_{i}\right)$ to denote the $\sigma_{j}$-axis intercept of $\mathcal{L}_{i}$.

The principal result is as follows.

THEOREM 3. We have the following.

(i) Assume that $\boldsymbol{x}$ is good for $(f, g)$, and $\mathcal{L}_{i}$ is a nonvertical line. Set $\sigma_{\iota}^{*}(\boldsymbol{x})=\max _{j}\left\{\sigma_{j}\left(\mathcal{L}_{i}\right)\right\}$. 
Let $\mathcal{M}_{\boldsymbol{\omega}}$ be an asymptotic monomial for $\mathbf{F}_{W}$ (see Definition 1.8). Then for any $\varepsilon \ll 1$,

$$
\mathcal{M}_{\boldsymbol{\omega}}^{*}(\boldsymbol{\lambda})=O_{\varepsilon}\left(|\boldsymbol{\lambda}|^{\sigma_{\iota}^{*}(\boldsymbol{x})+\varepsilon}\right) .
$$

If $\mathcal{L}_{i}$ is vertical, then $\mathcal{M}_{\boldsymbol{\omega}}^{*}(\boldsymbol{\lambda})=0$ if $\max \left\{\left|\lambda_{1}\right|,\left|\lambda_{2}\right|\right\} \gg 1$.

(ii) Assume that $\zeta_{k}:\left(F_{k}, G_{k}\right) \rightarrow(f, g)$ is an amelioration at $\boldsymbol{x}, \mathcal{L}_{i}$ is a nonvertical line, and $\mathcal{M}_{\boldsymbol{\omega}}$ is an asymptotic monomial for the fiber integral $\mathcal{F}_{W, k}$. Then for any $\varepsilon \ll 1$,

$$
\int \mathcal{M}_{\boldsymbol{\omega}}(\boldsymbol{\tau}) \Psi\left(\boldsymbol{\lambda} \cdot \zeta_{k}(\boldsymbol{\tau})\right)|d \boldsymbol{\tau}|_{\Omega_{k}}=O_{\varepsilon}\left(\left|\lambda_{k}\right|^{\sigma_{2}\left(\mathcal{L}_{i}\right)+\varepsilon}\right)
$$

If $\mathcal{L}_{i}$ is vertical, then this integral equals zero if $\left|\lambda_{k}\right| \gg 1$.

As in $\S 3$, Theorem 3 easily scales to bound $G_{W}(\boldsymbol{\lambda})$ in terms of a polygon from which one or both of the lines $\left\{s_{i}+1=0\right\}, i=1,2$, have been deleted. Using the above notation, define $\mathcal{U}=\left\{j \in[1, R]:\right.$ the $j$ th column of $\left.\mathbf{A} \neq \hat{\mathbf{e}}_{1}, \hat{\mathbf{e}}_{2}\right\}$. Write the $j$ th column as $\left(N_{j}, M_{j}, \mu_{j}\right)$. Set

$$
\Gamma^{*}=\partial\left(\bigcap_{j \in \mathcal{U}}\left\{\left(\sigma_{1}, \sigma_{2}\right) \in(-\infty, 0]^{2}: N_{j} \sigma_{1}+M_{j} \sigma_{2} \geqslant-\mu_{j}\right\}\right),
$$

and $\sigma\left(\Gamma^{*}\right)$ (respectively $\sigma_{2}\left(\Gamma^{*}\right)$ ) to denote the maximum of the axis intercepts of $\Gamma^{*}$ (respectively the $\sigma_{2}$-axis intercept of $\Gamma^{*}$, if it exists).

It can happen that $\sigma\left(\Gamma^{*}\right)$ or $\sigma_{2}\left(\Gamma^{*}\right)$ is considerably smaller than -1 . When this does occur, the bound in Theorem 4 represents a nontrivial improvement of Theorem 2. Examples of this were discovered in [Lic03].

Theorem 4. Assume the hypotheses in Theorem 3.

(i) If $\boldsymbol{x}$ is good for $(f, g)$ and $\Gamma^{*} \neq \emptyset$, then

$$
G_{W}(\boldsymbol{\lambda})=O_{\varepsilon}\left(|\boldsymbol{\lambda}|^{\sigma\left(\Gamma^{*}\right)+\varepsilon}\right) .
$$

If $\Gamma^{*}=\emptyset$, then $G_{W}(\boldsymbol{\lambda})=0$ if $\max \left\{\left|\lambda_{1}\right|,\left|\lambda_{2}\right|\right\} \gg 1$.

(ii) If $\left(F_{k}, G_{k}\right) \rightarrow(f, g)$ is an amelioration, and $\mathbf{A}=\mathbf{A}_{k}(\boldsymbol{x})$, then

$$
\left.G_{W}(\boldsymbol{\lambda})\right|_{\Omega_{k}}=O_{\varepsilon}\left(\left|\lambda_{k}\right|^{\sigma_{2}\left(\Gamma^{*}\right)+\varepsilon}\right) .
$$

If $\sigma_{2}\left(\Gamma^{*}\right)$ does not exist, then $\left.G_{W}(\boldsymbol{\lambda})\right|_{\Omega_{k}}=0$ if $\left|\lambda_{k}\right| \gg 1$.

\subsection{Proof of Theorem 3}

The proof of (4.1) will be given, and the very similar proof of (4.2) is left to the reader. The first ingredient required for the proof is the following.

Lemma 4.1. Assume that $\boldsymbol{x}$ is good for $(f, g)$ and $\hat{\mathbf{e}}_{2}$ is a column vector of $\mathbf{A}(\boldsymbol{x})$. Then $b_{2}=0$ for any $\boldsymbol{\omega}=(\iota, \mathbf{M}, \mathbf{b}, \boldsymbol{\chi}) \in \mathcal{Y}_{W}$.

Proof. By [Lic00, Proposition 5.2] and condition $P^{(2)}$, it follows that the polynomial $S=1-p^{-\left(1+s_{2}\right)}$ defines a component, with multiplicity one, of the polar divisor of the local zeta function for the wedge $W$,

$$
\mathbf{Z}_{\boldsymbol{x}}(\boldsymbol{\chi}, \mathbf{s})=\int_{U(\boldsymbol{x})-\{f \cdot g=0\}} \chi_{1}(\operatorname{ac} f) \chi_{2}(\operatorname{ac} g)|f|^{s_{1}}|g|^{s_{2}}\left|\theta^{*} d \mu\right| .
$$

Denoting the first two rows of $\mathbf{A}(\boldsymbol{x})$ by $\left(N_{1}, \ldots, N_{R}\right),\left(M_{1}, \ldots, M_{R}\right)$ with indices chosen so that $\left(N_{R}, M_{R}\right)=(0,1)$, it follows that $M_{j} / N_{j}<M_{R} / N_{R}={ }_{\text {def }}+\infty$ for any $j<R$. Thus, the vector $(0,1)$ is an 'extremal vector' of the lattice, denoted by $\mathcal{C}(\boldsymbol{x})$ [Lic00, p. 70]. Moreover, there exists a unique index $\ell$ so that $M_{\ell} / N_{\ell}=+\infty$. One can then apply Proposition 6.8 of [Lic00]. This ensures that the 


\section{B. LICHTIN}

multiplicity of $S$ does not increase beyond one in the partial fraction decomposition [Lic00, (5.2.1)] for $Z_{\boldsymbol{x}}(\chi, \mathrm{s})$. Since the partial fraction decomposition determines the set of vectors appearing in $\mathcal{Y}_{W}$, according to the recipe of $[\operatorname{Lic} 00, \S 6]$, it follows that for any $(\iota, \mathbf{M}, \mathbf{b}, \boldsymbol{\chi}) \in \mathcal{Y}_{W}$ it must be the case that $b_{2}=0$.

Remark 4.2. The same reasoning also shows that $b_{1}=0$ must occur if $\hat{\mathbf{e}}_{1}$ is a column vector of $\mathbf{A}(\boldsymbol{x})$. The argument also applies to the case in which $\boldsymbol{x}$ is bad and $\left(F_{k}, G_{k}\right) \rightarrow(f, g)$ is an amelioration. In this case, the data in $\mathcal{Y}_{W}$ are determined by a partial fraction decomposition for the local zeta function for $W$ of the map $\left(F_{k}, G_{k}\right)$ (i.e. replace $f, g$ by $F_{k}, G_{k}$ in the above integral). The conclusion is as follows: for any $(\iota, \mathbf{M}, \mathbf{b}, \boldsymbol{\chi}) \in \mathcal{Y}_{W}$, if $\hat{\mathbf{e}}_{2}$ is a column vector of $\mathbf{A}_{k}(\boldsymbol{x})$, then $b_{2}=0$.

The second ingredient in the proof of $(4.1)$ is as follows. Set $\chi_{0}$ to denote the trivial character on $\mathcal{U}_{p}$.

Lemma 4.3. If $(\iota, \mathbf{M}, \mathbf{b}, \boldsymbol{\chi}) \in \mathcal{Y}_{W}$ is such that $\chi_{2} \neq \chi_{0}$, then no side of $\Gamma_{\iota}$ lies on $\left\{\sigma_{2}+1=0\right\}$.

Proof. By [Lic00, (5.6.1)], the formula for the inverse Mellin transform implies that the proof of the lemma is a straightforward consequence of the following.

CLAim. If $\chi_{2} \neq \chi_{0}$ then $\mathbf{Z}_{\boldsymbol{x}}(\boldsymbol{\chi}, \mathbf{s})$ is not polar along $\left\{s_{2}+1=0\right\}$.

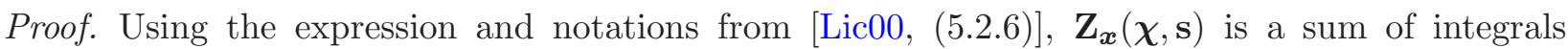
taken over finitely many cosets $\left[c+\left(p^{e}\right)^{n}\right]$ whose disjoint union equals $U(\boldsymbol{x})$. If the assertion is false, it follows that the integral over at least one such coset, say $\left[c^{\prime}+\left(p^{e}\right)^{n}\right]$, must be polar along $\left\{s_{2}+1=0\right\}$.

Let $\left(z_{1}, \ldots, z_{n}\right)$ be the centered (at $\left.\boldsymbol{x}\right)$ local coordinates on $U(\boldsymbol{x})$, such that the first $R$ columns of $\mathbf{A}(\boldsymbol{x})$ are nonzero (only) and the $R$ th column equals $\hat{\mathbf{e}}_{2}$. It then follows that the integral over $\left[c^{\prime}+\left(p^{e}\right)^{n}\right]$ equals the product of a nonzero constant $C=C\left(c^{\prime}, \chi\right)$ (subsequently dropped since it is not needed in the discussion) and

$$
\begin{aligned}
& \prod_{i=1}^{R} \int_{\left[c_{i}^{\prime}+\left(p^{e}\right)\right]} \chi_{1}^{N_{i}} \chi_{2}^{M_{i}}\left(\operatorname{ac} z_{i}\right)\left|z_{i}\right|^{N_{i} s_{1}+M_{i} s_{2}+\mu_{i}-1}\left|d z_{i}\right| \\
& \quad=\int_{\left[c_{R}^{\prime}+\left(p^{e}\right)\right]} \chi_{2}\left(\operatorname{ac} z_{R}\right)\left|z_{R}\right|^{s_{2}}\left|d z_{R}\right| \cdot\left(\prod_{i=1}^{R-1} \int_{\left[c_{i}^{\prime}+\left(p^{e}\right)\right]} \chi_{1}^{N_{i}} \chi_{2}^{M_{i}}\left(\operatorname{ac} z_{i}\right)\left|z_{i}\right|^{N_{i} s_{1}+M_{i} s_{2}+\mu_{i}-1}\left|d z_{i}\right|\right) .
\end{aligned}
$$

Since $\chi_{2} \neq \chi_{0}$, the evaluation of the integral with respect to $\left|d z_{R}\right|$ (see [Igu78, p. 89]) shows that this factor is an entire function in $s_{2}$. Moreover, since Condition $P^{(2)}$ is satisfied at $\boldsymbol{x}$, the polar locus of any other factor in the parentheses cannot be $\left\{s_{2}+1=0\right\}$. Thus, the integral could not be polar along $\left\{s_{2}+1=0\right\}$ whenever $\chi_{2} \neq \chi_{0}$. This proves the claim.

To finish the proof of Lemma 4.3 set $w_{i}=p^{-s_{i}}, i=1,2$, and use the expression obtained in [Lic00, (5.2.1), (6.6.1)]:

$$
\begin{aligned}
\mathbf{Z}_{\boldsymbol{x}}(\boldsymbol{\chi}, w) & =\sum_{L \subset\{1, \ldots, R\}} u_{L}(w, \chi) / v_{L}(w) \\
& =\sum_{L \subset\{1, \ldots, R\}} \sum_{\substack{\{i, j\} \in \mathcal{I}_{0} \\
\{i, j\} \subset L}} \frac{h_{i, j}(w, \boldsymbol{\chi})}{S_{i}^{k_{i}} S_{j}^{k_{j}}}+\sum_{\substack{i, \ell \\
i \in L}} \frac{h_{\ell}(w, \boldsymbol{\chi})}{S_{i}^{\ell}},
\end{aligned}
$$

where $S_{i}=1-p^{-\mu_{i}} w_{1}^{N_{i}} w_{2}^{M_{i}}, v_{L}(w)=\prod_{j \in L} S_{j}^{\nu_{j}}$, and each $\nu_{j}$ is a positive integer. If $\chi_{2} \neq \chi_{0}$, then the claim ensures that $S_{R}=1-p^{-1} w_{2}$ is not a factor of any $v_{L}(w)$ whenever $u_{L}(w, \chi) \neq 0$. Thus, $\chi_{2} \neq \chi_{0}$ implies that $S_{R}$ cannot appear as a factor in the denominator of any nonzero term on the second line of (4.3). Applying the operator Res ${ }_{w_{1}=0} \operatorname{Res}_{w_{2}=0}\left(\cdot w_{1}^{-\operatorname{ord} t_{1}-1} w_{2}^{- \text {ord } t_{2}-1}\right)$ to each fraction on the right-hand side determines the asymptotic monomials $\mathcal{M}_{\boldsymbol{\omega}}(\mathbf{t})$. One concludes that 


\section{On A QUESTION OF Igusa, II}

if $\boldsymbol{\omega}=(\iota, \mathbf{M}, \mathbf{b}, \boldsymbol{\chi}) \in \mathcal{Y}_{W}$, and $\chi_{2} \neq \chi_{0}$, then $R \notin \iota$. In other words, no such $\Gamma_{\iota}$ can have a side that lies on the line $\left\{\sigma_{2}+1=0\right\}$.

One now completes the proof of (4.1) as follows. For any $\boldsymbol{\omega}$ such that $\iota=\{i, R\} \in \mathcal{I}_{0}, \mathbf{b}=\left(b_{1}, 0\right)$, and $\chi=\left(\chi_{1}, \chi_{0}\right)$, it suffices to bound $\left.\mathcal{M}_{\boldsymbol{\omega}}^{*}(\boldsymbol{\lambda})\right|_{\Omega_{2}}$ in terms of $\sigma_{2}\left(\mathcal{L}_{i}\right)$. A similar bound in $\Omega_{1}$ is then argued in the same way (see Definition 2.2 and $\S 3$ ). Reindexing so that $i \rightarrow 1$ and $R \rightarrow 2$, the cone $\mathcal{C}_{\iota}=\left\langle\left(N_{1}, M_{1}\right),(0,1)\right\rangle_{\mathbb{Z}_{+}}$, with $\alpha=M_{1} / N_{1}$ and $\beta=+\infty$. Using the notation in $\S 2.1$, the elements of each effective slice $\mathcal{E}^{(i)}\left(r_{1}, \lambda_{2}\right)$, are determined in Lemma 2.3. Since $\left(N_{2}, M_{2}\right)=(0,1)$ it follows that $D^{\prime}=N_{1}^{\prime}=1$ and $Q\left(r_{1}, \epsilon_{2}\right)=r_{1}^{\prime} \eta+m_{2}-\epsilon_{2}\left(\right.$ recall that $\left.\epsilon_{2}=-\operatorname{ord} \lambda_{2}-\vartheta_{W}\right)$. Thus, the set of all possible $q$ equals $\left(-\infty, Q\left(r_{1}, \epsilon_{2}\right)\right]$, and the set of all $r_{2}$ values equals $\left[\epsilon_{2}, \infty\right) \cap \mathbb{N}$. Furthermore, when $\beta=+\infty$, this condition can only occur if $j \in\{2,3,5\}$ in Definition 2.4.

The next point is the crucial one. Recall from $\S 2.1$ that the monomial map $\mathbf{z} \in U(\boldsymbol{x}) \rightarrow \mathbf{z}^{\mathbf{M}}$ determines the range of the second component of the map $(f, g)=\mathbf{P} \circ \theta-\mathbf{P} \circ \theta(\boldsymbol{x})$. Thus, the set of angular component values of elements of the range of $\left.g\right|_{U(\boldsymbol{x})}$ equals

$$
\{\operatorname{ac} y: y=g(\mathbf{z})\}=\left\{u_{1}^{M_{1}} \cdots u_{n}^{M_{n}}: u_{1}, \ldots, u_{n} \in \mathcal{U}_{p}\right\} .
$$

Condition $P^{(2)}$ and the choice of indexing above now states that $u_{2}$ must be a factor of this monomial on $\mathcal{U}_{p}^{n}$. Thus, for any $u \in \mathcal{U}_{p}$ there exist units $u_{1}, \ldots, u_{n}$ such that $u_{1}^{M_{1}} u_{2} u_{3}^{M_{3}} \cdots u_{n}^{M_{n}}=u$. Writing $\mathbf{b}_{j}=\left(b_{j, 1}, b_{j, 2}\right)$ in Claim 1 , one concludes that the set $\left\{\mathbf{b}_{1}, \ldots, \mathbf{b}_{T}\right\}$ satisfies the following property:

$$
\bigsqcup_{j=1}^{T}\left[b_{j, 2}\right]_{2 \delta+1}=\mathcal{U}_{p}
$$

One now observes that if $j \in\{2,5\}$ and $r_{1} \in \mathcal{S}_{j}^{\prime}\left(\epsilon_{2}, \ell\right.$ ) (satisfying $N_{1} \mid r_{1}-m_{1}$ ) is such that ord $x=r_{1}$, then the fact that $v_{2}=-1$ implies

$$
\begin{aligned}
\int_{E^{\left(i_{j}\right)}\left(x, \lambda_{2}\right)} \Psi\left(\lambda_{2} y\right)|d y| & =\sum_{e \in \mathcal{E}^{\left(i_{j}\right)}\left(r_{1}, \lambda_{2}\right)} p^{-e} \sum_{b_{j, 2} \in \mathcal{U}_{p}} p_{\left(\bmod p^{2 \delta+1}\right)} p^{-2 \delta-1} \Psi\left(p^{e+\operatorname{ord} \lambda_{2}} b_{j, 2}\right) \\
& =\sum_{e \geqslant \epsilon_{2}} p^{-e} \int_{\mathcal{U}_{p}} \Psi\left(p^{e+\operatorname{ord} \lambda_{2}} u\right)|d u|=\int_{\left(p^{\epsilon_{2}}\right)} \Psi\left(\lambda_{2} w\right)|d w|=0,
\end{aligned}
$$

since $\epsilon_{2}+$ ord $\lambda_{2}=-\vartheta_{W}<0$ (see Corollary A.2 below).

Thus, the inner integral for $\mathcal{M}_{\boldsymbol{\omega}}^{*}$ can only be nonzero if $r_{1} \in \mathcal{S}_{j}^{\prime \prime}\left(\epsilon_{2}, \boldsymbol{\ell}\right)$ for some $j$ and $\boldsymbol{\ell} \in \mathcal{I}_{j}$. Since $\beta=+\infty$, Lemma 2.5 shows that $\mathcal{S}_{j}^{\prime \prime}\left(\epsilon_{2}, \ell\right) \neq \emptyset$ only occurs when $\alpha>0$ and $j \in\{2,3\}$. By Lemma 2.8(ii), the inner integral over $E^{\left(i_{j}\right)}\left(x, \lambda_{2}\right)$ is bounded (uniformly in $\lambda_{2}$ ) by $|x|^{-\alpha v_{2}}$ $\sum_{e} d_{e}^{\prime}(\ell) \log ^{e}|x|$. In addition, using the notation from Remark 2.7 to write the elements of $\mathcal{S}_{j}^{\prime \prime}\left(\epsilon_{2}, \ell\right)$, it follows that if ord $x \in \mathcal{S}_{j}^{\prime \prime}\left(\epsilon_{2}, \ell\right)$, then ord $x=c+u L$ for some $u \geqslant U_{j}$, where $c+U_{j} L=\epsilon_{2} / \alpha+O(1)$. Thus, the estimation argument from $\S 3$ shows that for any $e$ one has:

$$
\int_{\operatorname{ord} x \in \mathcal{S}_{j}^{\prime \prime}\left(\epsilon_{2}, \ell\right)}|x|^{-v_{1}-\alpha v_{2}-1} \log ^{b_{1}+e}|d x|=O_{\varepsilon}\left(\left|\lambda_{2}\right|^{v_{1} / \alpha+v_{2}+\varepsilon}\right)=O_{\varepsilon}\left(\left|\lambda_{2}\right|^{\sigma_{2}\left(\mathcal{L}_{1}\right)+\varepsilon}\right) .
$$

If $\mathcal{L}_{i}$ is vertical, this states that $\alpha=0$ so that $\mathcal{S}_{j}^{\prime \prime}\left(\epsilon_{2}, \boldsymbol{\ell}\right)=\emptyset$ for any $\boldsymbol{\ell}, j$. By the above argument, this ensures that $\left.\mathcal{M}_{\boldsymbol{\omega}}^{*}(\boldsymbol{\lambda})\right|_{\Omega_{2}}=0$ for $\epsilon_{2} \gg 1$. This completes the proof of (4.1).

\section{Appendix A. Evaluation of some one variable oscillatory integrals}

The evaluation of certain one variable oscillatory integrals are assembled here for the reader's convenience. First, one recalls the following result [Igu78, p. 56]. 


\section{B. LICHTIN}

Lemma A.1. If $R$ is any integer such that $R \leqslant-\operatorname{ord} \lambda-1$ and $\alpha>-1$, then

$$
\int_{(p)^{R}}|t|^{\alpha} \Psi(t \lambda)|d t|=p^{\alpha+1}\left[\frac{\left(1-p^{-1}\right) p^{-(\alpha+1)}}{1-p^{-(\alpha+1)}}-\frac{1}{p}\right]|\lambda|^{-\alpha-1} .
$$

A consequence of Lemma A.1 is important when $\alpha=0$.

Corollary A.2. If $\lambda$ satisfies ord $\lambda<-R$, then $\int_{(p)^{R}} \Psi(t \lambda)|d t|=0$.

A version of the above with an additional $\log ^{b}|t|$ factor in the integrands is also needed. The following suffices. It is proved using Lemma A.1 and partial summation.

LEMma A.3. We have the following.

(a) If $R+\operatorname{ord} \lambda \geqslant 0$ and $b, L \geqslant 1$, then for any $\alpha$,

$$
\int_{(p)^{R}-(p)^{R+L}}|t|^{\alpha} \log ^{b}|t| \Psi(t \lambda)|d t|=\sum_{i=0}^{b}\left(c_{i}^{\prime}(R+L+1)^{i} p^{-(R+L+1)(\alpha+1)}-c_{i} R^{i} p^{-R(\alpha+1)}\right),
$$

where each $c_{i}, c_{i}^{\prime}$ depends only on $p, b, \alpha$. Assuming that $\alpha>-1$ and letting $L \rightarrow \infty$, it follows that

$$
\int_{(p)^{R}}|t|^{\alpha} \log ^{b}|t| \Psi(t \lambda)|d t|=\sum_{i=0}^{b}\left(-c_{i}\right) R^{i} p^{-R(\alpha+1)} .
$$

(b) If $b, L \geqslant 1$, then for any $\alpha, \int_{(p)^{R}-(p)^{R+L}}|t|^{\alpha} \log ^{b}|t| \Psi(t \lambda)|d t|=0$ if $R+(L-1)+$ ord $\lambda \leqslant-1$. If $R+L+$ ord $\lambda \geqslant 0$ and $R \leqslant-1-\operatorname{ord} \lambda$, then

$$
\int_{(p)^{R}-(p)^{R+L}}|t|^{\alpha} \log ^{b}|t| \Psi(t \lambda)|d t|=\int_{(p)^{-1-\operatorname{ord} \lambda_{-}(p)^{R+L}}}|t|^{\alpha} \log ^{b}|t| \Psi(t \lambda)|d t| .
$$

\section{Appendix B. Proof of the assertion Remarks 1.4(ii)}

Although the argument is elementary, it is rather long, so it seems best to place the proof in this appendix. One chooses indexing so that $(i, k)=(1,2)$.

Assume that $\boldsymbol{z}$ are local coordinates on $U(\mathbf{x})$ (centered at $\mathbf{x}$ ) such that (see (1.1)):

$$
\begin{gathered}
F_{2}(\boldsymbol{z})=\prod_{i} z_{i}^{L_{i}} u_{1}(\boldsymbol{z}), \quad G_{2}(\boldsymbol{z})=\prod_{i} z_{i}^{M_{i}} u_{2}(\boldsymbol{z}), \quad \operatorname{rank}\left(\begin{array}{ccc}
L_{1} & \ldots & L_{n} \\
M_{1} & \ldots & M_{n}
\end{array}\right)=2, \\
\zeta_{2}\left(F_{2}, G_{2}\right)=\left(c F_{2}^{\gamma}, \psi\left(F_{2}\right)+G_{2}\right)=(f, g), \quad \text { where } \psi\left(F_{2}\right)=c_{\kappa} F_{2}^{\kappa}+c_{\kappa+1} F_{2}^{\kappa+1}+\cdots, c_{\kappa} \neq 0 .
\end{gathered}
$$

Since the rank of the matrix equals two, there exist coordinates $\mathbf{w}=\left(w_{1}, \ldots, w_{n}\right)$ such that if $\mathbf{L}=\left(L_{1}, \ldots, L_{n}\right)$ and $\mathbf{M}=\left(M_{1}, \ldots, M_{n}\right)$ then

$$
F_{2}(\mathbf{w})=\mathbf{w}^{\mathbf{L}}, \quad G_{2}(\mathbf{w})=\mathbf{w}^{\mathbf{M}} .
$$

Since $\mathbf{x}$ is bad for $(f, g)$ in the sense of (Remarks 1.2(iii)), it follows that $\mathbf{w}^{\kappa \mathbf{L}} \mid \mathbf{w}^{\mathbf{M}}$; that is, $\kappa L_{i} \leqslant M_{i}$ for each $i$, but strict inequality holds for at least one $i$. Choosing indexing so that $L_{1}>0$, define new coordinates $\boldsymbol{v}=\left(v_{1}, \ldots, v_{n}\right)$ by setting

$$
v_{1}=w_{1}\left(1+\frac{c_{\kappa+1}}{c_{\kappa}} \mathbf{w}^{\mathbf{L}}+\frac{c_{\kappa+2}}{c_{\kappa}} \mathbf{w}^{2 \mathbf{L}}+\cdots\right)^{1 / \kappa L_{1}}, \quad v_{i}=w_{i}, i \geqslant 2 .
$$

Writing $w_{1}=v_{1}(1+\varphi(\boldsymbol{v})), \varphi(\mathbf{0})=0$, one then observes that

$$
\varphi(\boldsymbol{v})=\Phi\left(\boldsymbol{v}^{\mathbf{L}}\right)
$$




\section{ON A QUESTION OF IGUSA, II}

This is verified by induction by showing that for each integer $\ell \geqslant 1$, there exists a polynomial $\varphi_{\ell}(t)$ such that

$$
\varphi(\boldsymbol{v}) \equiv \varphi_{\ell}\left(\boldsymbol{v}^{\mathbf{L}}\right) \quad\left(\bmod \left(v_{1}, \ldots, v_{n}\right)^{\ell|\mathbf{L}|}\right) .
$$

Since $\varphi(\boldsymbol{v})$ is analytic, the sequence $\varphi_{\ell}\left(\boldsymbol{v}^{\mathbf{L}}\right)$ converges to an analytic $\Phi\left(\boldsymbol{v}^{\mathbf{L}}\right)$ on some open compact $U_{1}(\mathbf{x})$. Thus,

$$
F_{2}^{\gamma}=\boldsymbol{v}^{\gamma \mathbf{L}}\left(1+\Phi\left(\boldsymbol{v}^{\mathbf{L}}\right)\right)^{\gamma L_{1}}, \quad \psi\left(\mathbf{F}_{2}\right)+G_{2}=c_{\kappa} \boldsymbol{v}^{\kappa \mathbf{L}}\left[1+c_{\kappa}^{-1} \cdot \boldsymbol{v}^{\mathbf{M}-\kappa \mathbf{L}} \cdot\left(1+\Phi\left(\boldsymbol{v}^{\mathbf{L}}\right)\right)^{M_{1}}\right] .
$$

Next, define the coordinates $\boldsymbol{y}=\left(y_{1}, \ldots, y_{n}\right)$, analytic in some $U_{2}(\mathbf{x})$, by setting

$$
y_{1}=v_{1} \cdot\left[1+c_{\kappa}^{-1} \cdot \boldsymbol{v}^{\mathbf{M}-\kappa \mathbf{L}} \cdot\left(1+\Phi\left(\boldsymbol{v}^{\mathbf{L}}\right)\right)^{M_{1}}\right]^{1 / \kappa L_{1}}, \quad y_{i}=v_{i}, i \geqslant 2 .
$$

It is then evident that $g(\boldsymbol{y})=c_{\kappa} \boldsymbol{y}^{\kappa \mathbf{L}}$. Now, writing

$$
\left[1+c_{\kappa}^{-1} \cdot \boldsymbol{v}^{\mathbf{M}-\kappa \mathbf{L}} \cdot\left(1+\Phi\left(\boldsymbol{v}^{\mathbf{L}}\right)\right)^{M_{1}}\right]^{1 / \kappa L_{1}}=1+\boldsymbol{v}^{\mathbf{M}-\kappa \mathbf{L}}\left(c+u\left(\boldsymbol{v}^{\mathbf{L}}\right)\right)
$$

where $c+u\left(\boldsymbol{v}^{\mathbf{L}}\right)$ is a unit in $U_{2}(\mathbf{x})$, it follows that

$$
\begin{gathered}
v_{1}=y_{1}\left(1+\boldsymbol{y}^{\mathbf{M}-\kappa \mathbf{L}} V(\boldsymbol{y})\right), \quad v_{i}=y_{i}, i \geqslant 2, \quad V=\text { an analytic unit in } U_{2}(\mathbf{x}) ; \\
F_{2}^{\gamma}(\boldsymbol{y})=\left.\boldsymbol{y}^{\gamma \mathbf{L}} \cdot\left(1+\boldsymbol{y}^{\mathbf{M}-\kappa \mathbf{L}} V(\boldsymbol{y})\right)^{\gamma L_{1}} \cdot\left(1+\Phi\left(\boldsymbol{v}^{\mathbf{L}}\right)\right)^{\gamma L_{1}}\right|_{v_{1}=y_{1}\left(1+\boldsymbol{y}^{\mathbf{M}-\kappa \mathbf{L}} V(\boldsymbol{y})\right)} .
\end{gathered}
$$

It is clear that one can write

$$
\left(1+\boldsymbol{y}^{\mathbf{M}-\kappa \mathbf{L}} V(\boldsymbol{y})\right)^{\gamma L_{1}}=1+\boldsymbol{y}^{\mathbf{M}-\kappa \mathbf{L}} V^{\prime} \quad \text { where } V^{\prime} \text { is an analytic unit on some } U_{3}(\mathbf{x}) .
$$

Either $\Phi\left(\boldsymbol{v}^{\mathbf{L}}\right)=0$, or there exists $j_{0} \geqslant 1$ such that $\Phi\left(\boldsymbol{v}^{\mathbf{L}}\right)=\sum_{j \geqslant j_{0}} e_{j} \boldsymbol{v}^{j \mathbf{L}}$ and $e_{j_{0}} \neq 0$. It also follows by an elementary manipulation that if $\Phi \neq 0$, then

$$
\begin{aligned}
\left.\left(1+\Phi\left(\boldsymbol{v}^{\mathbf{L}}\right)\right)^{\gamma L_{1}}\right|_{v_{1}=y_{1}\left(1+\boldsymbol{y}^{\mathbf{M}-\kappa \mathbf{L}} V(\boldsymbol{y})\right)} & =1+\sum_{j \geqslant j_{0}} e_{j} \boldsymbol{y}^{j \mathbf{L}}\left(1+\boldsymbol{y}^{\mathbf{M}-\kappa \mathbf{L}} V(\boldsymbol{y})\right)^{j L_{1}} \\
& =e\left(\boldsymbol{y}^{\mathbf{L}}\right)+\boldsymbol{y}^{\mathbf{M}-\left(\kappa-j_{0}\right) \mathbf{L}} \cdot V^{\prime \prime}(\boldsymbol{y}),
\end{aligned}
$$

where $e\left(\boldsymbol{y}^{\mathbf{L}}\right)$ and $V^{\prime \prime}(\boldsymbol{y})$ are analytic units on some $U_{4}(\mathbf{x})$.

Now define

$$
i_{0}= \begin{cases}0 & \text { if } \Phi=0 \\ j_{0} & \text { if } \Phi \neq 0 .\end{cases}
$$

An additional manipulation then shows the existence of an analytic $\psi_{1}(t)(\neq 0)$ of order $\gamma$ such that

$$
F_{2}^{\gamma}(\boldsymbol{y})=\psi_{1}\left(\boldsymbol{y}^{\mathbf{L}}\right)+\boldsymbol{y}^{\mathbf{M}+\left(\gamma-\kappa+i_{0}\right) \mathbf{L}} \cdot V^{\prime \prime \prime}
$$

where $V^{\prime \prime \prime}$ is an analytic unit on some $U_{5}(\mathbf{x})$. Setting $F_{1}=\boldsymbol{y}^{\mathbf{L}}, G_{1}=\boldsymbol{y}^{\left.\mathbf{M}+\left(\gamma-\kappa+i_{0}\right)\right) \mathbf{L}} \cdot V^{\prime \prime \prime}$, and defining $\zeta_{1}:\left(F_{1}, G_{1}\right) \rightarrow(f, g)$ by

$$
\zeta_{1}\left(F_{1}, G_{1}\right)=\left(\psi_{1}\left(F_{1}\right)+G_{1}, c_{\kappa} \cdot F_{1}^{\kappa \mathbf{L}}\right),
$$

it is clear that $\zeta_{1}$ is an amelioration of type 1 at $\mathbf{x}$. In addition, note that the role of $\gamma$ in (1.2c) is now played by $\kappa=\operatorname{ord}_{t} \psi(t)$.

\section{REFERENCES}

BK00 A. Braverman and D. Kazhdan, $\gamma$-functions of representations and lifting, Geom. Funct. Anal. (Special volume, Part 1) (2000), 237-278.

Clu04 R. Cluckers, Multivariate Igusa theory: Decay rates of exponential sums, Internat. Math. Res. Notices, to appear.

Cut02 S. D. Cutkosky, Monomialization of morphisms from 3-folds to surfaces, Lecture Notes in Mathematics, vol. 1786 (Springer, Berlin, 2002). 


\section{ON A QUESTION OF Igusa, II}

Den00 J. Denef, Arithmetic and geometric applications of quantifier elimination for valued fields, Math. Sci. Res. Inst. Publ., vol. 39 (Cambridge University Press, 2000), 173-198.

Igu73 J.-I. Igusa, Geometry of absolutely admissible representations, in Number theory, algebraic geometry and commutative algebra (in honor of Yasuo Akizuki) (Kinokuniya, Tokyo, 1973), 373-452.

Igu76 J.-I. Igusa, A Poisson formula and exponential sums, J. Fac. Sci. Univ. Tokyo Sect. IA Math. 23 (1976), 223-244.

Igu78 J.-I. Igusa, Forms of Higher Degree, Tata Institute Lectures, vol. 59 (Springer, Berlin, 1978).

Lic00 B. Lichtin, On a question of Igusa: Towards a theory of several variable asymptotic expansions I, Compositio Math. 120 (2000), 25-82.

Lic03 B. Lichtin, Uniform bounds for some exponential sums $\left(\bmod p^{r}\right)$ in two variables, in Proc. Session in Analytic Number Theory and Diophantine equations, January-June 2002, eds D. R. Heath-Brown and B. Z. Moroz, Bonner Mathematische Schriften, vol. 360 (University of Bonn, Bonn, 2003).

Lic04 B. Lichtin, Uniform bounds for two variable real oscillatory integrals and singularities of mappings, Preprint (2004).

Wei65 A. Weil, Sur la formule de Siegel dans la théorie des groupes classiques, Acta Math. 113 (1965), $1-87$.

Yam83 T. Yamazaki, Integrals defining singular series, Mem. Fac. Sci. Kyushu Univ. Ser. A 37 (1983), $113-128$.

Ben Lichtin lichtin@math.rochester.edu

49 Boardman Street, Rochester, NY 14607, USA 